\title{
The motivic Satake equivalence
}

\author{
Timo Richarz ${ }^{1}$. Jakob Scholbach ${ }^{2}$
}

Received: 3 April 2020 / Revised: 23 March 2021 / Accepted: 5 April 2021 / Published online: 15 May 2021

(c) The Author(s) 2021

\section{Abstract}

We refine the geometric Satake equivalence due to Ginzburg, Beilinson-Drinfeld, and Mirković-Vilonen to an equivalence between mixed Tate motives on the double quotient $L^{+} G \backslash L G / L^{+} G$ and representations of Deligne's modification of the Langlands dual group $\widehat{G}$.

\section{Contents}

1 Introduction . . . . . . . . . . . . . . . . . . . . . . . . 1596

2 Motives on affine flag varieties . . . . . . . . . . . . . . . . . . . . . . 1601

3 The convolution product . . . . . . . . . . . . . . . . . . . . . . . . 1611

4 Purity of Tate motives . . . . . . . . . . . . . . . . . . . . . . . . . . . . . . 1622

5 Mixed Tate motives on the affine Grassmannian . . . . . . . . . . . . . . . . . . . . . . 1625

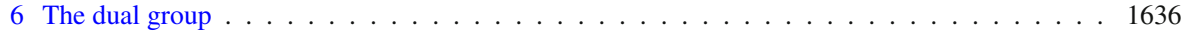

Appendix A: Complements on motives . . . . . . . . . . . . . . . . . . . . . . . . . . . . . 1644

References. . . . . . . . . . . . . . . . . . . . . . . . . . 1650

Communicated by Wei Zhang.

Research of T.R. partially funded by the Deutsche Forschungsgemeinschaft (DFG, German Research Foundation)-394587809. Resarch of J.S. funded by DFG, Sonderforschungsbereich 878 and DFG Cluster of Excellency "Mathematics Münster".

$凶$ Timo Richarz

richarz@mathematik.tu-darmstadt.de

Jakob Scholbach

jakob.scholbach@uni-muenster.de

1 Technische Universitat Darmstadt Fachbereich Mathematik, Darmstadt, Germany

2 Westfälische Wilhelms-Universität Münster, Mathematisches Institut, Münster, Germany 


\section{Introduction}

\subsection{Motivation and goals}

Split reductive groups are classified by their root data. These come in pairs, consisting of a root datum and its associated dual root datum. Accordingly, to every split reductive group $G$, there is associated its (Langlands) dual group $\widehat{G}$.

The work of Kazhdan and Lusztig [41,42] shows that the representation theory of $\widehat{G}$ is closely related to the singularities arising in certain orbit closures inside (affine) flag varieties associated to $G$. Building upon [50], the work of Ginzburg [30], BelinsonDrinfeld [8] and Mirković-Vilonen [52] revealed an equivalence of symmetric tensor categories between the category of finite-dimensional $\widehat{G}$-representations and the category of certain sheaves on an infinite-dimensional variety $\mathrm{Gr}_{G}$ known as the affine Grassmannian of $G$. This categorical equivalence is called the geometric Satake equivalence. It is an important tool in geometric representation theory which appears in different contexts and has a wide range of applications. For further details on the subject, the reader may refer to the notes of Baumann and Riche [7] and of Zhu [66], to $[57,61]$ for the relation with the classical Satake isomorphism (for which see [33]), and to [65] for a Satake equivalence in the case of mixed characteristic.

The goal of the present manuscript is to provide a motivic refinement of the geometric Satake equivalence. This has both philosophical and concrete consequences: the above papers devoted to the Satake equivalence use different base schemes, and also use different cohomology theories. It is therefore desirable to describe the common content of such different approaches, which is a goal accomplished in this paper. As far as concrete applications are concerned, let us point out that one of our main motivations is the work of Lafforgue [46] on the Langlands parametrization for global function fields. V. Lafforgue in particular conjectures [46, Conj. 12.12] that this parametrization is of motivic origin independent of an auxiliary prime number $\ell$ coming from the use of $\ell$-adic étale cohomology. A first evidence for Lafforgue's conjecture is the construction of intersection cohomology motives on moduli stacks of $G$-shtukas alias IC-Chow groups in [60]. The motivic Satake equivalence established in this paper is a second step of an ongoing project whose goal is to provide a motivic approach to V. Lafforgue's Langlands parametrization.

\subsection{Results}

Let $G$ be a Chevalley group over $\mathbf{Z}$ (=split reductive group scheme [18]), and fix $T \subset B \subset G$, a split maximal torus contained in a Borel subgroup over $\mathbf{Z}$. The loop group of $G$ is the group-valued functor on the category of rings $R$ given by $L G(R)=G(R((\varpi)))$. Its subgroup functor $L^{+} G(R)=G(R \llbracket \varpi \rrbracket)$ is the positive loop group. Here $R \llbracket \varpi \rrbracket \subset R((\varpi))$ denotes the ring of power series in a formal variable $\varpi$, contained in its Laurent series. For every finite field $\mathbf{F}_{q}$, the classical Satake isomorphism [33] is an isomorphism of $\mathbf{Q}(\sqrt{q})$-algebras

$$
\mathcal{C}_{c}\left(L^{+} G\left(\mathbf{F}_{q}\right) \backslash L G\left(\mathbf{F}_{q}\right) / L^{+} G\left(\mathbf{F}_{q}\right) ; \mathbf{Q}(\sqrt{q})\right) \simeq R_{\widehat{G}} \otimes_{\mathbf{Q}} \mathbf{Q}(\sqrt{q}),
$$


where $\sqrt{q}$ is a fixed square root of $q$ needed in the construction. The left hand side of (1.1) are thus $\mathbf{Q}(\sqrt{q})$-valued functions supported on finitely many double cosets. The convolution of such functions turns the left hand side into an algebra known as the spherical Hecke algebra. On the right hand side of (1.1) the group $\widehat{G}$ is the Langlands dual group of $G$ formed over $\mathbf{Q}$ (with respect to a fixed pinning). Then $R_{\widehat{G}}$ is the Grothendieck $\mathbf{Q}$-algebra of the category of representations of $\widehat{G}$ on finite-dimensional $\mathbf{Q}$-vector spaces. Its ring structure is given by the tensor product of representations. Writing $V_{\mu}$ for the simple $\widehat{G}$-representation of highest weight $\mu$, where $\mu \in X_{*}(T)^{+}$ is a dominant cocharacter, their classes $\left[V_{\mu}\right]$ form a $\mathbf{Q}$-basis of $R_{\widehat{G}}$. Under (1.1), these correspond to functions which are related to the singularities of an infinite-dimensional space as follows. The affine Grassmannian is the étale sheaf quotient

$$
\mathrm{Gr}_{G} \stackrel{\text { def }}{=}\left(L G / L^{+} G\right)^{\text {et }},
$$

which is representable by an ind-projective ind-scheme (=infinite union of projective $\mathbf{Z}$-schemes) equipped with a left action of $L^{+} G$. For each dominant cocharacter $\mu \in$ $X_{*}(T)^{+}$, we denote by $\mathrm{Gr}_{G}^{\leq \mu}$ the scheme-theoretic image of the orbit map $L^{+} G \rightarrow$ $\mathrm{Gr}_{G}, g \mapsto g \cdot \varpi^{\mu} \cdot e$ where $e \in \mathrm{Gr}_{G}(\mathbf{Z})$ is the base point. Then $\mathrm{Gr}_{G}^{\leq \mu} \rightarrow \operatorname{Spec}(\mathbf{Z})$ is a projective scheme, usually singular, which contains the open smooth $L^{+} G$-orbit $\mathrm{Gr}_{G}^{\mu} \subset \mathrm{Gr}_{G}^{\leq \mu}$ as a fiberwise dense open subscheme. There is a presentation on the underlying reduced locus

$$
\left(\mathrm{Gr}_{G}\right)_{\mathrm{red}}=\operatorname{colim}_{\mu \in X_{*}(T)^{+}} \mathrm{Gr}_{G}^{\leq \mu}
$$

For a finite field $\mathbf{F}_{q}$ and each auxiliary prime $\ell \nmid q$, let $\mathrm{IC}_{\mu, q, \ell}$ be the $\ell$-adic intersection complex of $\mathrm{Gr}_{G}^{\leq \mu} \otimes \mathbf{z} \mathbf{F}_{q}$ in the sense of Goresky-MacPherson-Deligne. A surprising observation of [50] is that the class $\left[V_{\mu}\right]$ corresponds under (1.1) (up to a power of $\sqrt{q}$ ) to the trace of Frobenius function of $\mathrm{IC}_{\mu, q, \ell}$ given by Grothendieck's sheaf function dictionary.

The geometric Satake equivalence is a categorification of (1.1). It is known in several settings using different cohomology theories: in [7,30,52] the authors work with $\mathrm{Gr}_{G} \otimes \mathbf{Z} \mathbf{C}$ using Betti cohomology (the latter two with more general coefficients however), whereas [8] works with $\mathrm{Gr}_{G} \otimes \mathbf{z} \mathbf{C}$ using $D$-modules, and [57,61] works with $\mathrm{Gr}_{G} \otimes \mathbf{z} k$ for general fields $k$ using $\ell$-adic étale cohomology. Here we provide a motivic refinement.

In analogy with the left hand side of (1.1) we consider the double quotient $L^{+} G \backslash L G / L^{+} G \rightarrow \operatorname{Spec}(\mathbf{Z})$ viewed as a groupoid-valued functor on the category of rings. For each such functor we have constructed in [60] a category of motives (with rational coefficients)

$$
\operatorname{DM}\left(L^{+} G \backslash L G / L^{+} G\right)=\operatorname{DM}\left(L^{+} G \backslash L G / L^{+} G ; \mathbf{Q}\right) .
$$

The collection of all such categories is equipped with a Grothendieck six functor formalism (with certain restrictions on the $*$-pullback). The construction in op. cit. builds upon the recent advances in the theory of motivic sheaves due to Ayoub $[2,3,5]$ and Cisinski-Déglise $[14,15]$ as envisioned by Beilinson. 
In there we consider a much smaller full subcategory of stratified Tate motives

$$
\operatorname{DTM}\left(L^{+} G \backslash L G / L^{+} G\right) \subset \operatorname{DM}\left(L^{+} G \backslash L G / L^{+} G\right) .
$$

This category is generated by all Tate twists $1_{\mathrm{Gr}_{G}^{\mu}}(n), n \in \mathbf{Z}$ of the characteristic relative motives of the orbits $\mathrm{Gr}_{G}^{\mu}, \mu \in X_{*}(T)^{+}$, and is well suited for applications to Hecke algebras. It is equipped with a convolution product: namely, for two motives $A, B$ on the double quotient their convolution is defined as the motive

$$
A \star B=m_{!} p^{!}(A \otimes B)
$$

using the maps

$$
L^{+} G \backslash L G / L^{+} G \times L^{+} G \backslash L G / L^{+} G \stackrel{p}{\longleftarrow} L^{+} G \backslash L G \times L^{+^{+}} G L / L^{+} G \stackrel{m}{\longrightarrow} L^{+} G \backslash L G / L^{+} G,
$$

where $p$ is the canonical projection and $m$ is induced by the multiplication $L G \times L G \rightarrow$ $L G,\left(g_{1}, g_{2}\right) \mapsto g_{1} \cdot g_{2}$. The convolution of motives in (1.2) is modeled on the convolution in the spherical Hecke algebra (1.1). (The use of the functor $p^{!}$in (1.3), as opposed to $p^{*}$, is related to the construction of (1.2). Elements of this category should be more appropriately thought of as "measures" instead of "functions" which leads in a categorical setting to the use of !-pullback instead of $*$-pullback.)

The fibers of convolution morphisms are paved by cells [34] which leads to the following result.

Theorem A If A, B are stratified Tate motives (resp. and pure of some weight), then $A \star B$ is again stratified Tate (resp. and pure) (3.17), (4.8).

In fact, we show a more general version of Theorem A where $L^{+} G$ is replaced by an arbitrary parahoric subgroup of $L G$. As a consequence, the category of stratified Tate motives is equipped with a monoidal structure with respect to the convolution. We now cut out an abelian subcategory as follows.

By [60], which extends the work of Soergel and Wendt [63], for each $\mu \in X_{*}(T)^{+}$, $n \in \mathbf{Z}$ there exists an intersection motive

$$
\operatorname{IC}_{\mu, \mathbf{Z}}(n) \in \operatorname{DTM}\left(L^{+} G \backslash L G / L^{+} G\right)
$$

which is supported on $\mathrm{Gr}_{G}^{\leq \mu}$ and such that $\left.\mathrm{IC}_{\mu, \mathbf{Z}}(n)\right|_{\mathrm{Gr}_{G}^{\mu}}=1_{\mathrm{Gr}_{G}^{\mu}}(n)$. We emphasize that the non-trivial construction of $\operatorname{IC}_{\mu, \mathbf{Z}}(n)$ in [60] bypasses the use of standard conjectures on $t$-structures of triangulated categories of motives. For any finite field $\mathbf{F}_{q}$ and each prime $\ell \nmid q$, its base change $\mathrm{IC}_{\mu, \mathbf{F}_{q}}:=\left.\mathrm{IC}_{\mu, \mathbf{Z}}\right|_{\mathbf{F}_{q}}$ maps under the $\ell$ adic realization to the intersection complex $\mathrm{IC}_{\mu, q, \ell}$ on $\mathrm{Gr}_{G}^{\leq \mu} \otimes_{\mathbf{Z}} \mathbf{F}_{q}$ as above. For the field of rational numbers $\mathbf{Q}$, its base change $\mathrm{IC}_{\mu, \mathbf{Q}}:=\left.\mathrm{IC}_{\mu, \mathbf{Z}}\right|_{\mathbf{Q}}$ maps under the Betti realization to the intersection complex on the stratified topological space $\operatorname{Gr}_{G}^{\leq \mu}(\mathbf{C})$. Thus, the motives $\mathrm{IC}_{\mu, \mathbf{Z}}$ interpolate between various intersection sheaves arising in 
the literature on the Satake equivalence, both in the sense of letting the base scheme vary, and also in the sense of varying the cohomology theory.

Again by [60], the category $\operatorname{DTM}\left(L^{+} G \backslash L G / L^{+} G\right)$ is equipped with a nondegenerate (perverse) motivic $t$-structure. Its heart, the abelian subcategory of mixed (stratified) Tate motives

$$
\operatorname{MTM}\left(L^{+} G \backslash L G / L^{+} G\right) \subset \operatorname{DTM}\left(L^{+} G \backslash L G / L^{+} G\right),
$$

is generated by the intersection motives (1.4). Using motivic (global) cohomology provides a $\mathbf{Q}$-linear functor $\omega: \operatorname{MTM}\left(L^{+} G \backslash L G / L^{+} G\right) \rightarrow \operatorname{Vect}_{\mathbf{Q}}$, see Definition 5.11 .

Theorem B (i) If A, B are mixed Tate motives, then their convolution $A \star B$ is mixed Tate as well (5.8)

(ii) The motivic cohomology functor $\omega$ is $\mathbf{Q}$-linear, exact, faithful and equipped with functorial isomorphisms $\omega(A \star B) \simeq \omega(A) \otimes_{\mathbf{Q}} \omega(B)$ for all objects $A, B$. (5.13)

(iii) There exists a unique symmetric monoidal structure on $\operatorname{MTM}\left(L^{+} G \backslash L G / L^{+} G\right)$ with respect to the convolution product $\star$ characterized by the property that $\omega$ is a tensor functor with respect to (ii) and the canonical symmetric monoidal structure on $\left(\operatorname{Vect}_{\mathbf{Q}}, \otimes\right)(5.9,5.14)$

Already for $G=\mathrm{GL}_{2}$, the subcategory of non-twisted intersection motives $\mathrm{IC}_{\mu}$, $\mu \in X_{*}(T)^{+}$is not stable under convolution. As observed in [37, Rmk. 2.10] and [61] this phenomenon is linked to the presence of $\sqrt{q}$ in (1.1) and leads to the appearance of a $\mathbf{G}_{m, \mathbf{Q}}$-extension on the dual side.

We consider the central subgroup $\boldsymbol{\mu}_{2} \subset \widehat{G} \times \mathbf{G}_{m, \mathbf{Q}}$ of order 2 generated by the element $(\epsilon,-1)$ where $\epsilon:=(2 \rho)(-1) \in \widehat{G}(\mathbf{Q})$ and $2 \rho$ denotes the sum of positive roots viewed as a cocharacter of $\widehat{G}$. Deligne's modified Langlands dual group (see [20], [24, §2], [13, §5], and [66, 5.5.14]) is defined as the split reductive Q-group

$$
\widehat{G}_{1} \stackrel{\text { def }}{=} \widehat{G} \times \boldsymbol{\mu}_{2} \mathbf{G}_{m, \mathbf{Q}} .
$$

The extra $\mathbf{G}_{m, \mathbf{Q}}$ factor corresponds to the occurrence of Tate twists when forming the convolution of intersection motives. We denote by $\operatorname{Rep}_{\mathbf{Q}}\left(\widehat{G}_{1}\right)$ the category of algebraic $\widehat{G}_{1}$-representations on $\mathbf{Q}$-vector spaces. This category is semi-simple. Its simple objects are labelled by $V_{\mu}(n)$ with $\mu \in X_{*}(T)^{+}, n \in \mathbf{Z}$.

To make the connection with (1.1) we base change the groups $L G_{\mathbf{F}_{q}}:=L G \otimes_{\mathbf{Z}} \mathbf{F}_{q}$ and $L^{+} G_{\mathbf{F}_{q}}:=L^{+} G \otimes_{\mathbf{Z}} \mathbf{F}_{q}$ to a finite field. Then the analogue of Theorem B holds with $\operatorname{MTM}\left(L^{+} G \backslash L G / L^{+} G\right)$ replaced by $\operatorname{MTM}\left(L^{+} G_{\mathbf{F}_{q}} \backslash L G_{\mathbf{F}_{q}} / L^{+} G_{\mathbf{F}_{q}}\right)$.

Theorem C For each finite field $\mathbf{F}_{q}$, there is an equivalence of symmetric monoidal categories

$$
\operatorname{MTM}\left(L^{+} G_{\mathbf{F}_{q}} \backslash L G_{\mathbf{F}_{q}} / L^{+} G_{\mathbf{F}_{q}}\right) \simeq \operatorname{Rep}_{\mathbf{Q}}\left(\widehat{G}_{1}\right), \quad \operatorname{IC}_{\mu, \mathbf{F}_{q}}(n) \mapsto V_{\mu}(n),
$$

using the tensor structure from Theorem B. Under this equivalence, the motivic cohomology functor $\omega$ corresponds to the forgetful functor $\operatorname{Rep}_{\mathbf{Q}}\left(\widehat{G}_{1}\right) \rightarrow \operatorname{Vect}_{\mathbf{Q}}$. For each 
prime $\ell \nmid q$, this equivalence gives under the $\ell$-adic étale realization the geometric Satake equivalence as explained in [66, 5.5.14]. $(6.8,6.12)$

Among other things, Theorem $\mathrm{C}$ asserts that the left hand category is semisimple. This semi-simplicity is inferred, via Lusztig's parity vanishing, from the semi-simplicity of the abelian category $\operatorname{MTM}\left(\mathbf{F}_{q}\right)$. The latter semi-simplicity holds since higher algebraic $K$-theory of $\mathbf{F}_{q}$ is torsion by Quillen's computation, see Example 6.12. This semi-simplicity is then lifted to the mixed Tate motives on the double quotient over a finite field. Passing to the trace of the Frobenius function as in, say, [16] one recovers the Satake isomorphism similar to (1.1) where one now considers Q-valued functions and a quotient of the representation ring $R_{\widehat{G}_{1}}$, cf. Sect. 6.4.

In contrast to $\operatorname{MTM}\left(\mathbf{F}_{q}\right)$, the categories $\operatorname{MTM}(\mathbf{Z})$ and, a fortiori, $\operatorname{MTM}\left(L^{+} G \backslash L G / L^{+} G\right)$ are no longer semi-simple. More generally, if $S$ is a sufficiently nice scheme which satisfies the Beilinson-Soulé vanishing (e.g. the spectrum of finite fields as above; number fields or their rings of integers; function fields over a finite field or their rings of integers; or filtered colimits of these rings) the category of mixed Tate motives

$$
\operatorname{MTM}\left(L^{+} G_{S} \backslash L G_{S} / L^{+} G_{S}\right)
$$

is well-defined and satisfies Theorem B where we denote $L^{(+)} G_{S}:=L^{(+)} G \times \operatorname{Spec}(\mathbf{Z}) S$. In the category (1.5) we also have the intersection motives $\operatorname{IC}_{\mu, S}(n)$ for $\mu \in X_{*}(T)^{+}$, $n \in \mathbf{Z}$. We denote by $\mathrm{Sat}_{G, S}$ the full semi-simple subcategory of (1.5) generated by the intersection motives by means of direct sums. This subcategory $\operatorname{Sat}_{G, S}$ is stable under convolution, and hence inherits a symmetric monoidal structure.

Theorem D Let $p: S \rightarrow \operatorname{Spec}(\mathbf{Z})$ be a base scheme as above.

(i) The pullback of motives induces an equivalence of symmetric monoidal categories

$$
\operatorname{Sat}_{G, \mathbf{Z}} \rightarrow \operatorname{Sat}_{G, S}, \quad \mathrm{IC}_{\mu, \mathbf{Z}}(n) \mapsto p^{*} \mathrm{IC}_{\mu, \mathbf{Z}}(n)=\mathrm{IC}_{\mu, S}(n),
$$

and hence $\operatorname{Sat}_{G, S} \simeq \operatorname{Rep}_{\mathbf{Q}}\left(\widehat{G}_{1}\right)$ by Theorem $C$ independently of $S$ (6.6)

(ii) Let $\mathscr{U}_{S}$ be the pro-unipotent algebraic $\mathbf{Q}$-group arising from extensions in the category $\operatorname{MTM}(S)$. Then there is an equivalence of symmetric monoidal categories

$$
\operatorname{MTM}\left(L^{+} G_{S} \backslash L G_{S} / L^{+} G_{S}\right) \simeq \operatorname{Rep}_{\mathbf{Q}}\left(\mathscr{U}_{S} \rtimes \widehat{G}_{1}\right)
$$

where on the right-hand side is the category of representations of the proalgebraic group $\mathscr{U}_{S} \rtimes \widehat{G}_{1}$ on $\mathbf{Q}$-vector spaces $(6.14,6.15)$

Part (i) of Theorem D precisely formulates the experimental fact that under the geometric Satake isomorphism the dual side does not depend on the base scheme over which the affine Grassmannian is defined. Part (ii) is, in part, an extension of Levine's work [47] which one recovers in the special case where $G$ is the trivial group. 


\subsection{Related and future work}

Zhu [67] has sketched the construction of a motivic Satake equivalence over $\mathbf{F}_{q}$ using the category of numerical motives of Jannsen. Zhu's approach is based on an explicit enumeration of algebraic cycles on affine Grassmannians. By comparison, the approach taken in this paper is more strongly relying on the general framework of motives, which we expect to be fruitful also for our upcoming work.

One may imagine using the theory of Nori motives to produce an abelian category of motives related to the Satake equivalence. Nori motives, however, depend upon the cohomology theory chosen at the outset. In the case of motives over $\mathbf{F}_{q}$, say, this would in practice mean choosing $\ell$-adic cohomology for some $\ell$ prime to $q$. Again, the choice of working with motives as developed by Ayoub and CisinskiDéglise is based on the desire to apply it to a Langlands parametrization over function fields, where we precisely seek to avoid a reference to $\ell$-adic cohomology.

Throughout this paper, motives have rational coefficients. Using upcoming work of Spitzweck on t-structures on Tate motives with integral coefficients, it would be very interesting to establish a Satake equivalence in this situation. The reader is referred to [68] for a result on the level of functions.

There are versions of the geometric Satake equivalence using different affine Grassmannians such as the Witt vector (or $p$-adic) affine Grassmannian of Zhu [65], the $B_{\mathrm{dR}}$-affine Grassmannian of Fargues-Scholze [23]. In subsequent work [59], we extend the methods of this paper to cover a Satake equivalence for Witt vector Grassmannians.

As was stated above, we conceive the results in [60] and the Satake equivalence in this paper to be two steps in a long-term program aiming to prove a motivic version of V. Lafforgue's Langlands parametrization over function fields. The immediate next step, to be addressed in a subsequent paper, is to improve on Theorem $\mathrm{C}$ by proving a motivic version of Gaitsgory's factorization (or fusion) version of the geometric Satake equivalence [27]. This will require suitable Whitney-Tate properties of BeilinsonDrinfeld Grassmannians, as opposed to the affine Grassmannian $\mathrm{Gr}_{G}$ encountered above. Here the six functor formalism for the categories of motives mentioned in Theorem $\mathrm{C}$ will be crucial. Further steps in this program include a motivic Drinfeld lemma, a motivic construction of excursion operators, and their identification with Hecke operators. All these remain to be done as well.

\section{Motives on affine flag varieties}

In this section, we recollect and extend some material from [60] as is needed throughout this manuscript. In Sect. 2.1, we state some facts on loop groups and their affine flag varieties. The next Sect. 2.2 treats motives on prestacks which is applied in Sects. 2.32.4 to affine flag varieties. Section 2.5 gathers some facts pertaining to KazhdanLusztig parity vanishing. 
Notation 2.1 Throughout this manuscript, $S$ is an irreducible, regular scheme which is separated of finite type over a Noetherian, excellent, separated and at most 2dimensional scheme. Further, we assume that $S$ satisfies the Beilinson-Soulé vanishing conjecture (cf. [60, (3.2.2)]), and admits an $\ell$-adic realization functor in the sense of [60, §2.1.2, Rmk. 3.2.9].

Examples include finite fields, number fields and function fields of curves over finite fields, their rings of algebraic integers and filtered colimits of these rings.

\subsection{Loop Groups and their affine flag varieties}

We refer the reader to [60, §4] for further details and references on the following material.

We denote by $\operatorname{AffSch}_{S}$ the category of affine schemes $\operatorname{Spec}(R) \rightarrow S$ equipped with a map to $S$. Let $G$ be a split reductive group scheme over $S$, for example $G=\mathrm{GL}_{n, S}$. The loop group $L G$ is the presheaf

$$
L G: \operatorname{AffSch}_{S}^{\text {op }} \rightarrow \text { Groups, } \operatorname{Spec}(R) \mapsto G(R((\varpi))),
$$

where $R((\varpi))$ is the ring of Laurent series in the formal variable $\varpi$. It is represented by an ind-affine ind-scheme over $S$, and in particular $L G$ is an fpqc sheaf on $\operatorname{AffSch}_{S}^{\text {op }}$.

We fix $T \subset B \subset G$ over $S$, a split maximal torus contained in a Borel subgroup. Let $\mathscr{A}=\mathscr{A}(G, B, T)$ be the standard apartment with origin 0 defined by $G$ and standard alcove a defined by $B$. We only consider facets $\mathbf{f} \subset \mathscr{A}$ which are contained in the closure of $\mathbf{a}$. Attached to $\mathbf{f}$ is the parahoric subgroup $\mathcal{P}_{\mathbf{f}} \subset L G$ which is an $S$-affine, $S$-flat closed subgroup scheme. For this paper, the most important case is $\mathbf{f}=0$, in which case $\mathcal{P}_{0}=: L^{+} G$ is the positive loop group given by the presheaf

$$
L^{+} G: \operatorname{AffSch}_{S}^{\text {op }} \rightarrow \text { Groups, } \operatorname{Spec}(R) \mapsto G(R \llbracket \varpi \rrbracket)
$$

If $\mathbf{f}=\mathbf{a}$, then $\mathcal{P}_{\mathbf{a}}=: \mathcal{B}$ is the standard Iwahori subgroup defined as the preimage of $B$ under the map $L^{+} G \rightarrow G, \varpi \mapsto 0$.

The étale sheafification of the quotient $\mathrm{Fl}_{\mathbf{f}}:=\left(L G / \mathcal{P}_{\mathbf{f}}\right)^{\text {et }}$ is called the partial affine flag variety associated with $\mathbf{f}$. It is represented by an ind-projective ind-scheme over $S$. For $\mathbf{f}=0$, it is denoted by $\mathrm{Gr}=\mathrm{Gr}_{G}$, and called the affine Grassmannian.

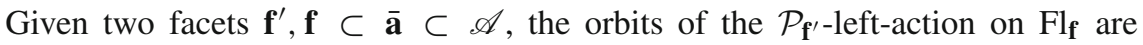
enumerated by the double quotient $W_{\mathbf{f}^{\prime}} \backslash W / W_{\mathbf{f}}$ of the Iwahori-Weyl (or extended affine Weyl) group $W=W(G, T)$ by the subgroups $W_{\mathbf{f}^{\prime}}, W_{\mathbf{f}} \subset W$ generated by the reflections preserving $\mathbf{f}^{\prime}$ resp. $\mathbf{f}$. The choice of a defines a length function $l=l\left(\mathbf{f}^{\prime}, \mathbf{f}\right): W_{\mathbf{f}^{\prime}} \backslash W / W_{\mathbf{f}} \rightarrow \mathbf{Z}_{\geq 0}$, and a Bruhat partial order $\leq$ on the double coset. For

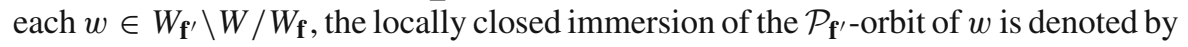

$$
\iota_{w}: \mathrm{Fl}_{\mathbf{f}}^{w} \stackrel{j_{w}}{\hookrightarrow} \mathrm{Fl}_{\mathbf{f}}^{\leq w} \stackrel{i_{w}}{\hookrightarrow} \mathrm{Fl}_{\mathbf{f}}
$$

Then $\mathrm{Fl}_{\mathbf{f}}^{\leq w} \rightarrow S$ is a proper scheme called the (affine) Schubert scheme. It contains $\mathrm{Fl}_{\mathbf{f}}^{w}$ as an open $S$-smooth subscheme which is fibrewise dense and which is called 
the (affine) Schubert cell. For each map $\operatorname{Spec}(k) \rightarrow S$ from a field, the base change $\mathrm{Fl}_{\mathbf{f}}^{w} \times{ }_{S} \operatorname{Spec}(k) \subset \mathrm{Fl}_{\mathbf{f}}^{\leq w} \times{ }_{S} \operatorname{Spec}(k)$ identifies on the underlying reduced locus with the Schubert cell, resp. Schubert variety over $k$ attached to the class $w$ and the $k$ group scheme $G \times{ }_{S} \operatorname{Spec}(k)$. Further, each $\mathrm{Fl}_{\mathbf{f}}^{w} \rightarrow S$ is pure of relative dimension $l(w)$ and $\mathrm{Fl}_{\mathbf{f}}^{v} \subset \mathrm{Fl}_{\mathbf{f}}^{\leq w}$ if and only if $v \leq w$ for $v, w \in W_{\mathbf{f}^{\prime}} \backslash W / W_{\mathbf{f}}$. If $\mathbf{f}^{\prime}=\mathbf{f}=0$, then $W_{0} \backslash W / W_{0}=X_{*}(T)^{+}$is the partially ordered set of dominant cocharacters with length function $l=l(0,0): X_{*}(T)^{+} \rightarrow \mathbf{Z}_{\geq 0}, \mu \mapsto\langle 2 \rho, \mu\rangle$ where $\rho$ denotes the half sum of the $B$-positive roots and $\langle-,-\rangle: X^{*}(T) \times X_{*}(T) \rightarrow \mathbf{Z}$ is the natural pairing. In the case of the affine Grassmannian, we denote the orbits (resp. orbit closures) by $\mathrm{Gr}^{\mu} \subset \mathrm{Gr} \leq \mu$ for $\mu \in X_{*}(T)^{+}$.

\subsection{Motives on prestacks}

We refer the reader to $[60, \S 2]$ for further details and references on the following material.

We consider the triangulated category of motives with rational coefficients

$$
\operatorname{DM}(X) \stackrel{\text { def }}{=} \operatorname{DM}(X, \mathbf{Q}), \quad X \in \operatorname{Sch}_{S}^{\mathrm{ft}},
$$

where $\operatorname{Sch}_{S}^{\mathrm{ft}}$ is the category of finite type schemes over $S$. This category is denoted by $\mathrm{DA}(X, \mathbf{Q})$ in [5] and by $\mathrm{D}_{\mathbf{A}^{1}, \text { et }}(X, \mathbf{Q})$ in [15]. Categories of motives with rational coefficients admit a full six functor formalism: there are pairs of adjoint functors $\left(f^{*}, f_{*}\right),\left(f_{!}, f^{!}\right)$for a map $f \in \operatorname{Sch}_{S}^{\mathrm{ft}}$ and $(-\otimes-, \operatorname{Hom}(-,-))$ satisfying the usual compatibilities such as smooth/proper base change, Poincaré duality, Künneth/projection formula etc. Following Hoyois [38] and Khan [43], this can be upgraded to a presheaf of $\infty$-categories

$$
\mathrm{DM}^{!}:\left(\mathrm{Sch}_{S}^{\mathrm{ft}}\right)^{\mathrm{op}} \rightarrow \mathrm{DGCat}_{\text {cont }}, \quad X \mapsto \mathrm{DM}(X), \quad f \mapsto f^{!},
$$

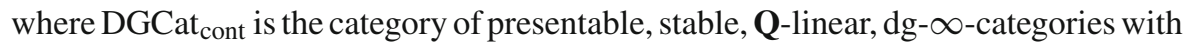

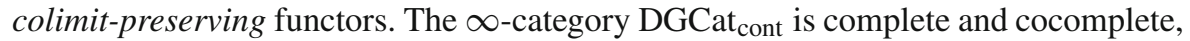
i.e., admits all (homotopy) limits and (homotopy) colimits, so that the following Kan extensions are available.

Definition 2.4 (i) Let $\mathrm{AffSch}_{S}^{\mathrm{ft}} \subset \mathrm{AffSch}_{S}$ be the full subcategory of objects of finite type over $S$. Throughout, we will replace this category by a small skeleton containing the objects of interest to us.

(ii) Fix some regular cardinal $\kappa$, and let $\operatorname{AffSch}_{S}^{\kappa}:=\operatorname{Pro}_{\kappa \text {-small }}\left(\mathrm{AffSch}_{S}^{\mathrm{ft}}\right)$ be the category of $\kappa$-small pro-objects in $\mathrm{AffSch}_{S}^{\mathrm{ft}}$.

(iii) The $\infty$-category of prestacks is $\operatorname{PreStk}_{S}^{\kappa}:=\operatorname{Fun}\left(\left(\operatorname{AffSch}_{S}^{\kappa}\right)^{\mathrm{op}}, \infty-\mathrm{Gpd}\right)$ where $\infty$-Gpd is the $\infty$-category of $\infty$-groupoids (also called spaces). Hereafter, we will usually drop the $\kappa$ from the notation so that PreStk $:=\operatorname{PreStk}_{S}^{\kappa}, \operatorname{AffSch}_{S}:=$ $\operatorname{AffSch}_{S}^{\kappa}$.

(iv) Define the functor

$$
\mathrm{DM}^{!}: \operatorname{AffSch}_{S}^{\text {op }} \rightarrow \text { DGCat }_{\text {cont }}
$$


to be the left Kan extension of the functor $\mathrm{DM}^{!}$in (2.3) along the inclusion $\mathrm{AffSch}_{S}^{\mathrm{ft}} \subset \mathrm{AffSch}_{S}$.

(v) Define the functor

$$
\mathrm{DM}^{!}: \operatorname{PreStk}_{S}^{\mathrm{op}} \rightarrow \text { DGCat }_{\mathrm{cont}}
$$

to be the right Kan extension of the preceding functor along the Yoneda embedding $\operatorname{AffSch}_{S} \subset \operatorname{PreStk}_{S}$.

We emphasize that $\mathrm{DM}^{!}$in (2.5) encodes a category of motives $\operatorname{DM}(X)$ (with rational coefficients) for each prestack $X$, and for each map $f: X \rightarrow Y$ in $\operatorname{PreStk}_{S}$ a colimit-preserving functor $f^{!}: \operatorname{DM}(Y) \rightarrow \operatorname{DM}(X)$. This definition follows the approach of Gaitsgory-Rozenblyum and Raskin. We refer to [60, §2.2] for references and also for further discussion of the definition.

Theorem 2.6 (i) The presheaf $\mathrm{DM}^{!}: \operatorname{PreStk}_{S}^{\mathrm{op}} \rightarrow$ DGCat $_{\text {cont }}$ is a sheaf in the étale topology. For each prestack $X \in \operatorname{PreStk}_{S}$ the $\infty$-sheafification $X \rightarrow X^{\mathrm{et}}$ induces an equivalence on categories of motives $\mathrm{DM}\left(X^{\mathrm{et}}\right) \stackrel{\simeq}{\longrightarrow} \mathrm{DM}(X), c f .[60$, Thm. 2.2.16].

(ii) The restriction of the presheaf $\mathrm{DM}^{!}$to the category of strict ind-schemes of indfinite type over $S$ admits a full six functor formalism (with certain restrictions on $\left.f^{*}\right), c f$. [60, Thm. 2.2.4].

\subsection{Stratified Tate motives on affine flag varieties}

We recall some material pertaining to stratified Tate motives, referring to $[60, \S 5]$ for further details.

By virtue of Definition 2.4, there is the category of motives $\operatorname{DM}\left(\mathcal{P}_{\mathbf{f}^{\prime}} \backslash L G / \mathcal{P}_{\mathbf{f}}\right)$ for each pair of facets $\mathbf{f}^{\prime}, \mathbf{f} \subset \overline{\mathbf{a}} \subset \mathscr{A}$. Using Theorem 2.6 (i), we have a forgetful functor

$$
\operatorname{DM}\left(\mathcal{P}_{\mathbf{f}^{\prime}} \backslash L G / \mathcal{P}_{\mathbf{f}}\right) \simeq \operatorname{DM}\left(\mathcal{P}_{\mathbf{f}^{\prime}} \backslash \mathrm{Fl}_{\mathbf{f}}\right) \rightarrow \operatorname{DM}\left(\mathrm{Fl}_{\mathbf{f}}\right)
$$

which associates to each motive on the double quotient its underlying non-equivariant motive.

Definition 2.7 (i) The category of $\left(\mathbf{f}^{\prime}, \mathbf{f}\right)$-stratified Tate motives $\mathrm{DTM}\left(\mathrm{Fl}_{\mathbf{f}}\right) \subset$ $\operatorname{DM}\left(\mathrm{Fl}_{\mathbf{f}}\right)$ is the full subcategory consisting of objects $M \in \mathrm{DM}\left(\mathrm{Fl}_{\mathbf{f}}\right)$ such that for all $w \in W_{\mathbf{f}^{\prime}} \backslash W / W_{\mathbf{f}}$

$$
\iota_{w}^{*} M \in \mathrm{DTM}\left(\mathrm{Fl}_{\mathbf{f}}^{w}\right)
$$

where $\iota_{w}$ is as in (2.2) and $\mathrm{DTM}\left(\mathrm{Fl}_{\mathbf{f}}^{w}\right) \subset \mathrm{DM}\left(\mathrm{Fl}_{\mathbf{f}}^{w}\right)$ denotes the subcategory generated by $1(n), n \in \mathbf{Z}$ under arbitrary shifts and colimits. (This condition is equivalent to requiring $\iota_{w}^{!} M \in \mathrm{DTM}\left(\mathrm{Fl}_{\mathbf{f}}^{w}\right)$ for all $w$, cf. [60, Def. 3.1.11, Cor. 4.3.12].)

(ii) The category of $\left(\mathbf{f}^{\prime}, \mathbf{f}\right)$-stratified equivariant Tate motives

$$
\operatorname{DTM}\left(\mathcal{P}_{\mathbf{f}^{\prime}} \backslash L G / \mathcal{P}_{\mathbf{f}}\right) \subset \operatorname{DM}\left(\mathcal{P}_{\mathbf{f}^{\prime}} \backslash L G / \mathcal{P}_{\mathbf{f}}\right)
$$


is the full subcategory consisting of objects $M$ whose underlying non-equivariant motive lies in $\operatorname{DTM}\left(\mathrm{Fl}_{\mathbf{f}}\right)$. There is a forgetful functor $\operatorname{DTM}\left(\mathcal{P}_{\mathbf{f}^{\prime}} \backslash L G / \mathcal{P}_{\mathbf{f}}\right) \rightarrow$ $\operatorname{DTM}\left(\mathrm{Fl}_{\mathbf{f}}\right)$.

The category $\mathrm{DTM}\left(\mathrm{Fl}_{\mathbf{f}}\right) \subset \mathrm{DM}\left(\mathrm{Fl}_{\mathbf{f}}\right)$ agrees by [60, Thm. 5.1.1] with the full subcategory generated by all $\iota_{w, !} 1(n)$ (resp. by all $\iota_{w, *} 1(n)$ ), and so is well-suited for applications to Hecke algebras.

Theorem 2.8 ([60, Thm. 5.3.4]) Let $S$ be a scheme as in Notation 2.1.

(i) The category $\mathrm{DTM}\left(\mathrm{Fl}_{\mathbf{f}}\right)$ carries the so-called perverse motivic $t$-structure whose heart is denoted by $\mathrm{MTM}\left(\mathrm{Fl}_{\mathbf{f}}\right)$. The subcategory $\mathrm{MTM}\left(\mathrm{Fl}_{\mathbf{f}}\right)^{\mathrm{c}}$ of compact objects is Artinian and Noetherian. Its simple objects are precisely the intersection motives on the orbit closures

$$
\mathrm{IC}_{w}(n) \stackrel{\text { def }}{=}\left(\iota_{w}\right)_{! *} 1(n)\left[d_{w}\right] \stackrel{\text { def }}{=} i_{w, !} j_{w, ! *} 1(n)\left[d_{w}\right], \quad n \in \mathbf{Z}, w \in W_{\mathbf{f}} \backslash W / W_{\mathbf{f}}
$$

where $\iota_{w}=i_{w} \circ j_{w}$ is as in (2.2) and $d_{w}$ is the relative dimension of $\mathrm{Fl}_{\mathbf{f}}^{w}$ over $S$.

(ii) If $\mathbf{f}^{\prime}=\mathbf{f}$, then the forgetful functor

$$
\operatorname{DTM}\left(\mathcal{P}_{\mathbf{f}} \backslash L G / \mathcal{P}_{\mathbf{f}}\right) \rightarrow \operatorname{DTM}\left(\mathrm{Fl}_{\mathbf{f}}\right)
$$

and the $t$-structure in (i) create a t-structure on the left hand category. The induced functor on the hearts $\operatorname{MTM}\left(\mathcal{P}_{\mathbf{f}} \backslash L G / \mathcal{P}_{\mathbf{f}}\right) \rightarrow \operatorname{MTM}\left(\mathrm{Fl}_{\mathbf{f}}\right)$ is fully faithful and induces a bijection on simple objects.

We point out the following results which are needed in Sect. 5 below.

Lemma 2.9 For $w \in W_{\mathbf{f}^{\prime}} \backslash W / W_{\mathbf{f}}$, there is an equivalence

$$
\operatorname{MTM}(S) \stackrel{\simeq}{\longrightarrow} \operatorname{MTM}\left(\mathrm{Fl}_{\mathbf{f}}^{w}\right)
$$

Proof The structure map $\mathrm{Fl}_{\mathbf{f}}^{w} \rightarrow S$ is smooth, and the Schubert cell admits a stratification into affine spaces, by virtue of the stratification in Iwahori orbits, cf. [60, Prop. 4.3.9]. Hence, this lemma follows from the general Lemma 2.10 below.

Lemma 2.10 Let $\pi: X \rightarrow S$ be a smooth surjective map of schemes of relative dimension $d$ with connected fibers. We assume that $X$ admits a stratification in the sense of [60, Def. 3.1.1] by schemes of the form $\mathbb{V}(\mathcal{E})$ (where $\mathcal{E}$ is a vector bundle over $S$ ), e.g., by affine spaces over $S$. Then there is an equivalence of categories

$$
\pi^{!}[-d]=\pi^{*}[d](d): \operatorname{MTM}(S) \stackrel{\simeq}{\longrightarrow} \operatorname{MTM}(X),
$$

where the Tateness of motives on $X$ is with respect to the stratification by a single stratum. 
Proof By the conventions in Notation 2.1, $S$ is connected and hence so is $X$ [64, Tag 0378]. The functor is fully faithful by [60, Lem. 3.2.12]. For essential surjectivity, we first claim that $\operatorname{Hom}_{S}(M, N[1])=\operatorname{Hom}_{X}\left(\pi^{*} M, \pi^{*} N[1]\right)$ for $M, N \in \operatorname{MTM}(S)$. We prove this by induction on the number of strata in $X$. If $X=\mathbb{V}(\mathcal{E})$ is a single stratum, then this holds even for all $N \in \operatorname{DTM}(S)$. For the inductive step we use as in loc. cit. the localization sequence for a minimal stratum

$$
Z=\mathbb{V}(\mathcal{E}) \stackrel{i}{\rightarrow} X \stackrel{j}{\leftarrow} U:=X \backslash Z
$$

Let $\pi_{Z}:=\pi \circ i, \pi_{U}:=\pi \circ j$. Since $X$ is connected and is assumed to have at least two strata, the codimension $c:=\operatorname{codim}_{X} Z$ is positive. By induction, the composite

$$
\operatorname{Hom}_{S}(M, N[1]) \rightarrow \operatorname{Hom}_{X}\left(\pi^{*} M, \pi^{*} N[1]\right) \rightarrow \operatorname{Hom}_{U}\left(\pi_{U}^{*} M, \pi_{U}^{*} N[1]\right)
$$

is an isomorphism. By the localization sequence, the kernel of the right hand map is mapped onto by $\operatorname{Hom}_{Z}\left(\pi_{Z}^{*} M, \pi_{Z}^{*} N(-c)[1-2 c]\right)$ which vanishes by the BeilinsonSoule condition for $Z$ (equivalently, for $S$ ). Hence the left hand map above is an isomorphism as well, showing our claim.

The generators $1(n)[d], n \in \mathbf{Z}$ of $\operatorname{MTM}(X)$ trivially lie in the image of our functor, so we are done by using that $\pi^{*}$ is an isomorphism on the level of extensions of mixed Tate motives by the above claim.

\subsection{Changing the base scheme}

For facets $\mathbf{f}^{\prime}, \mathbf{f} \subset \overline{\mathbf{a}} \subset \mathscr{A}$, we show that the category $\operatorname{DTM}\left(\mathrm{Fl}_{\mathbf{f}}\right)$ for the stratification in left- $\mathcal{P}_{\mathbf{f}^{\prime} \text {-orbits }}$ is, to a certain extent, insensitive to the choice of the base scheme $S$, cf. Theorem 2.14 below. In Sect. 6.1, we will sharpen this idea by introducing the (abelian) Satake category $\operatorname{Sat}_{G} \subset \mathrm{DTM}\left(\mathrm{Gr}_{G}\right)$ and showing that this category is completely independent of the base scheme $S$.

Let $f: T \rightarrow S$ be a map of schemes, where $T$ is Noetherian and of finite Krull dimension, so that $f^{*}: \operatorname{DM}(S) \rightarrow \operatorname{DM}(T)$ is well-defined. (An important example to have in mind is $T=\operatorname{Spec} \mathbf{F}_{p} \rightarrow S=\operatorname{Spec} \mathbf{Z}$.) We indicate base changes to $T$ by a subscript, e.g., $G_{T}:=G \times{ }_{S} T$. We still write $f$ for all maps obtained using such base changes, e.g., $f: \mathrm{Fl}_{\mathbf{f}, T} \rightarrow \mathrm{Fl}_{\mathbf{f}, S}$. The condition in [60, Thm. 2.4.2] is satisfied, so that we obtain a functor

$$
f^{*}: \operatorname{DM}\left(\mathrm{Fl}_{\mathbf{f}, S}\right) \rightarrow \operatorname{DM}\left(\mathrm{Fl}_{\mathbf{f}, T}\right)
$$

As before, write $\iota_{w}: \mathrm{Fl}_{\mathbf{f}}^{w} \rightarrow \mathrm{Fl}_{\mathbf{f}}$ for the inclusion of the $\mathcal{P}_{\mathbf{f}^{\prime}}$-orbits, both over $S$ and over $T$. We clearly have an equivalence $\left(\iota_{w}\right)^{*} f^{*} \stackrel{\simeq}{\rightarrow} f^{*}\left(\iota_{w}\right)^{*}$ by functoriality, so that $f^{*}$ restricts to a functor

$$
f^{*}: \operatorname{DTM}\left(\mathrm{Fl}_{\mathbf{f}, S}\right) \rightarrow \operatorname{DTM}\left(\mathrm{Fl}_{\mathbf{f}, T}\right) .
$$

Here is the key lemma concerning the change of the base scheme. 
Lemma 2.12 Let $w \in W_{\mathbf{f}^{\prime}} \backslash W / W_{\mathbf{f}}$. The following natural transformations of functors, when restricted to the indicated categories of Tate motives,

$$
\begin{aligned}
& f^{*}\left(\iota_{w}\right)_{*} \rightarrow\left(\iota_{w}\right)_{*} f^{*} \text { on } \operatorname{DTM}\left(\mathrm{Fl}_{\mathbf{f}, S}^{w}\right) \\
& \left(\iota_{w}\right) ! f^{*} \rightarrow f^{*}\left(\iota_{w}\right) \text { ! on } \operatorname{DTM}\left(\mathrm{Fl}_{\mathbf{f}, S}^{w}\right) \\
& f^{*}\left(\iota_{w}\right)^{!} \rightarrow\left(\iota_{w}\right)^{!} f^{*} \text { on } \operatorname{DTM}\left(\mathrm{Fl}_{\mathbf{f}, S}\right)
\end{aligned}
$$

are equivalences.

Proof The claim for $\left(\iota_{w}\right)$ ! results from base change. The claim for $\left(\iota_{w}\right)^{!}$will follow from the others using an induction argument based on the localization fiber sequence

$$
i^{!} \rightarrow i^{*} \rightarrow i^{*} j_{*} j^{*}
$$

for any complementary closed (resp. open) embedding $i$ (resp. $j$ ).

In order to show that $f^{*}$ commutes with $\left(\iota_{w}\right)_{*}$, we may assume that $\mathbf{f}^{\prime}=\mathbf{a}$ (the base alcove), since the stratification by $\mathcal{P}_{\mathbf{f}^{\prime}}$-orbits is coarser than the one by Iwahori orbits so that the claim for the Iwahori stratification together with a localization argument implies the one for the stratification by $\mathcal{P}_{\mathbf{f}^{\prime} \text {-orbits. }}$

We first show the claim for $\mathbf{f}=\mathbf{a}$. By [60, Prop. 5.2.2], $\mathrm{DTM}\left(\mathrm{Fl}_{\mathbf{a}}\right)$ is the smallest cocomplete full subcategory of $\mathrm{DM}\left(\mathrm{Fl}_{\mathbf{a}}\right)$ which contains the twists of the unit motives supported at the base points $\{\tau\}$ for each $\tau \in \operatorname{Stab}_{\mathbf{a}} \subset W$ and which is stable under the operation $\pi_{s}^{!} \pi_{s, !}$ along the smooth proper projection maps $\pi_{s}: \mathrm{Fl}_{\mathbf{a}} \rightarrow \mathrm{Fl}_{s}:=\mathrm{Fl}_{\mathbf{f}_{s}}$ for all simple reflections $s \in \mathbb{S}$. We proceed by induction on the length of $w$, the case $l(w)=0$ being trivial since $\iota_{w}$ is a closed embedding of a base point $\tau$ in this case. For $l(w)>0$, let $w=v \cdot s$ be a reduced expression with $s \in \mathbb{S}$. We obtain a fibre sequence

$$
\left(\iota_{v}\right)_{*} 1 \rightarrow \pi_{s}^{!} \pi_{s, !}\left(\iota_{v}\right)_{*} 1 \rightarrow\left(\iota_{w}\right)_{*} 1
$$

which is the dual of the fibre sequence [60, (5.1.2)].

Using induction $l(v)<l(w)$, the functor $f^{*}$ commutes with $\left(l_{v}\right)_{*}$. Since $\pi_{s}$ is smooth, $f^{*}$ also commutes with $\pi_{s}^{!}$, and hence with $\left(\iota_{w}\right)_{*}$ by (2.13). This finishes the case $\mathbf{f}=\mathbf{a}$.

Now, for a general facet $\mathbf{f} \subset \overline{\mathbf{a}}$, we reduce the claim to the one previously considered using the map $\pi: \mathrm{Fl}_{\mathbf{a}} \rightarrow \mathrm{Fl}_{\mathbf{f}}$. This map is smooth, proper, surjective and a stratified map with respect to the Iwahori stratification on both ind-schemes [60, Lem. 4.3.13]. Therefore, in the cartesian diagram

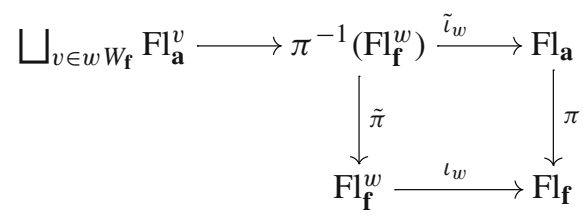


the preimage $\pi^{-1}\left(\mathrm{Fl}_{\mathbf{f}}^{w}\right)$ is itself stratified by some Iwahori strata on $\mathrm{Fl}_{\mathbf{a}}$ as indicated above. Using that $\pi$ is smooth, $\left(\tilde{l}_{w}\right)_{*} \tilde{\pi}^{*}=\pi^{*}\left(\iota_{w}\right)_{*}$. Moreover, $\pi^{*}$ commutes with $f^{*}$. Finally, $\pi^{*}$ is conservative. Thus, to show that $f^{*}$ commutes with $\left(\iota_{w}\right)_{*}$, we may replace the inclusion $\iota_{w}$ (both on $T$ and on $S$ ) by $\tau_{w}$. Using again a localization argument, we then reduce this statement to the one for the inclusions $\iota_{v}: \mathrm{Fl}_{\mathbf{a}}^{v} \rightarrow \mathrm{Fl}_{\mathbf{a}}$ of the Iwahori strata in the flag variety refining the preimage stratification under $\pi$.

Recall from [60, Thm. 2.4.2] that both categories carry weight structures. The aim of this section is to prove:

Theorem 2.14 Let $f: T \rightarrow S$ be a map of schemes both satisfying the conditions in Notation 2.1. Then the functor (2.11) has the following properties:

(i) it is conservative.

(ii) it creates weights, i.e., $M \in \mathrm{DTM}\left(\mathrm{Fl}_{\mathbf{f}, S}\right)$ is of weights $\geq n($ resp. $\leq n)$ iff $f^{*} M$ has the corresponding property.

(iii) it creates the $t$-structure, i.e., $M \in \operatorname{DTM}\left(\mathrm{Fl}_{\mathbf{f}, S}\right)$ is of in the " $\geq n$ " (resp. " $\leq n$ ”) part of the $t$-structure iff $f^{*} M$ has the corresponding property.

We need some preparation for the proof.

Proposition 2.15 In the situation of Theorem 2.14, the functor (2.11) is t-exact with respect to the perverse motivic $t$-structures (cf. Theorem 2.8), and commutes with the intermediate extension functors $\left(j_{w}\right)_{\text {!* }}$ defined in (2.2). In particular,

$$
f^{*}\left(\operatorname{IC}_{w, S}(n)\right)=\operatorname{IC}_{w, T}(n), \quad n \in \mathbf{Z}, w \in W_{\mathbf{f}^{\prime}} \backslash W / W_{\mathbf{f}} .
$$

Proof For both base schemes $S$ and $T$, the subcategory $\operatorname{DTM}\left(\mathrm{Fl}_{\mathbf{f}}\right)^{\leq 0}$ consists by definition precisely of those objects $M$ such that $\iota^{*} M \in \operatorname{DTM}\left(\mathrm{Fl}_{\mathbf{f}}^{+}\right)^{\leq 0}$ where $\iota: \mathrm{Fl}_{\mathbf{f}}^{+}=\sqcup_{w} \mathrm{Fl}_{\mathbf{f}}^{w} \rightarrow \mathrm{Fl}_{\mathbf{f}}$ denotes the disjoint union of the inclusions of all strata. Likewise with " $\geq 0$ " and $\iota$ ! instead. To show the exactness of $f^{*}$ we may by Lemma 2.12 replace $f$ by the induced map $f^{+}: \mathrm{Fl}_{\mathbf{f}, T}^{+} \rightarrow \mathrm{Fl}_{\mathbf{f}, S}^{+}$. It then remains to observe that the following diagram is cartesian and has smooth vertical maps

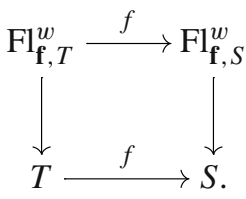

Thus the $t$-exactness of $f^{*}$ on the base implies the one for $f^{*}: \operatorname{DTM}\left(\mathrm{Fl}_{\mathbf{f}, S}^{w}\right) \rightarrow$ $\operatorname{DTM}\left(\mathrm{Fl}_{\mathbf{f}, T}^{w}\right)$ since the hearts of these $t$-structures are generated by the objects $1(n)\left[d_{w}\right]$ where $d_{w}=l(w)$ is the dimension of $\mathrm{Fl}_{\mathbf{f}}^{w}$ relative to the base scheme (which is the same for $S$, resp. $T$ ). The remaining claim now follows from Lemma 2.12 which ensure that $f^{*}$ commutes with all functors involved in the formation of $j_{w, ! *}:=\operatorname{im}\left({ }^{\mathrm{p}} \mathrm{H}^{0}\left(j_{w, !}\right) \rightarrow^{\mathrm{p}}\right.$ $\left.\mathrm{H}^{0}\left(j_{w, *}\right)\right)$. 
Proof of Theorem 2.14 For (i), let $M \in \mathrm{DTM}\left(\mathrm{Fl}_{\mathbf{f}, S}\right)$. For the conservativity, we have to show $f^{*} M=0$ implies $M=0$. Using the non-degeneracy of the perverse motivic t-structure [60, Cor. 3.2.6] and the t-exactness of $f^{*}$, it is enough to show the conservativity of $\left.f^{*}\right|_{\operatorname{MTM}\left(\mathrm{Fl}_{\mathbf{f}, S}\right)}$. Any $M \in \mathrm{MTM}\left(\mathrm{Fl}_{\mathbf{f}, S}\right)$ is the filtered colimit of its compact subobjects, so we may assume $M$ is also compact. Then, $M$ has a Jordan-Hölder series with simple constituents given by twisted intersection motives [60, 3.3.8]. We may thus assume that $M$ is an intersection motive, so we are done by (2.16). For (ii), we need to show that $f^{*}$ is weight-exact and detects weights. As in the proof of Proposition 2.15 , to show that $f^{*}$ is weight-exact, we may replace $\mathrm{Fl}_{\mathbf{f}}$ by $\mathrm{Fl}_{\mathbf{f}}^{+}$over both base schemes $S$ and $T$, which is again clear by definition of the weight structures. The detection of weights then follows from Lemma 2.17 below using part (i). For (iii), we use likewise the conservativity and $t$-exactness of $f^{*}$.

Lemma 2.17 A conservative, weight-exact functor $F: \mathcal{C} \rightarrow \mathcal{D}$ between triangulated categories with weight structures detects weights: if $F(M)$ has weights $<n$ (resp. $\geq n$ ) for some $M \in \mathcal{C}$, then the same is true for $M$.

Proof We use that $M$ has weights $\geq n$ (resp. $<n$ ) iff for any weight truncation triangle $E: M_{<n} \stackrel{s_{<n}}{\rightarrow} M \stackrel{s_{\geq n}}{\rightarrow} M_{\geq n}$, the maps $s_{\geq n}$ (resp. $s_{<n}$ ) are isomorphisms. Indeed, the " $\Leftarrow$ " direction holds by definition, the converse also follows from elementary applications of the axioms, see [25, Cor. 2.2.6, Cor. 2.2.7]. Given a weight truncation triangle $E$ for $M, F(E)$ is a weight truncation triangle for $F(M)$ by assumption. Hence our claim follows since $F$ is conservative.

\subsubsection{Pullback functoriality for equivariant motives}

Lemma 2.18 The functor $f^{*}$ descends to a functor

$$
\bar{f}^{*}: \operatorname{DM}\left(\mathcal{P}_{\mathbf{f}^{\prime}, S} \backslash L G_{S} / \mathcal{P}_{\mathbf{f}, S}\right) \rightarrow \operatorname{DM}\left(\mathcal{P}_{\mathbf{f}^{\prime}, T} \backslash L G_{T} / \mathcal{P}_{\mathbf{f}, T}\right) .
$$

This functor $\bar{f}^{*}$ preserves the subcategories of equivariant Tate motives.

Proof By construction, $f^{*}$ is the unique functor which is given by the usual $f^{*}$ on the level of finite type $S$-schemes and compatible with the insertion functors $\mathrm{DM}\left(\mathrm{Fl}_{\mathbf{f}}^{\leq w}\right) \rightarrow$ $\operatorname{DM}\left(\mathrm{Fl}_{\mathbf{f}}\right)$ (both over $S$ and $T$ ). By [60, Cor. 2.3.4], it is therefore enough to construct a functor

$$
\bar{f}^{*}: \operatorname{DM}\left(\mathcal{P}_{\mathbf{f}^{\prime}, S} \backslash \mathrm{Fl}_{\mathbf{f}, S}^{\leq w}\right) \rightarrow \operatorname{DM}\left(\mathcal{P}_{\mathbf{f}^{\prime}, T} \backslash \mathrm{Fl}_{\mathbf{f}, T}^{\leq w}\right)
$$

There is a split pro-unipotent subgroup $U \subset \mathcal{P}_{\mathbf{f}^{\prime}}$ such that the quotient $K:=\mathcal{P}_{\mathbf{f}^{\prime}} / U$

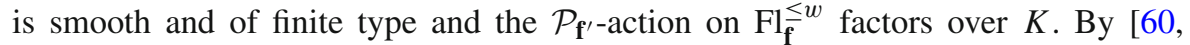
Prop. 2.2.11], $\mathrm{DM}\left(\mathcal{P}_{\mathbf{f}^{\prime}} \backslash \mathrm{Fl}_{\mathbf{f}}^{\leq w}\right) \cong \operatorname{DM}\left(K \backslash \mathrm{Fl}_{\mathbf{f}}^{\leq w}\right)$. Finally, in order to check the existence of $\bar{f}^{*}$ on this level, it is enough to observe that the maps in the bar construction $\operatorname{Bar}\left(K, \mathrm{Fl}^{\leq w}\right)$ are all smooth, and hence !-pullback along them commutes with $f^{*}$. Hence the $f^{*}$-functors in all levels of the diagram $\operatorname{DM}^{!}\left(\operatorname{Bar}\left(K, \mathrm{Fl}_{\mathbf{f}}^{\leq w}\right)\right)$ glue to a functor on the limit of this diagram, which is $\mathrm{DM}\left(K \backslash \mathrm{Fl}_{\mathbf{f}}^{\leq w}\right)$. 
Given that the underlying non-equivariant functor of $\bar{f}^{*}$ is just $f^{*}$, the preservation of equivariant Tate motives follows from (2.11).

\subsection{Kazhdan-Lusztig parity vanishing}

We now apply Proposition 2.15 to prove the Kazhdan-Lusztig parity vanishing [42, Thm. 5.5] (see also [50, Thm. 11.c)]) for the intersection motives. Our main tool is the $\ell$-adic realization functor which exists by assumption on $S$ (Notation 2.1). We continue with the notation and assumptions from Sect. 2.4. In particular, we fix two facets $\mathbf{f}, \mathbf{f}^{\prime}$ contained in the closure of the standard alcove $\mathbf{a}$, and denote by $\mathrm{DTM}\left(\mathrm{Fl}_{\mathbf{f}}\right)$ the category of $\left(\mathbf{f}^{\prime}, \mathbf{f}\right)$-stratified Tate motives whose heart is the abelian category $\operatorname{MTM}\left(\mathrm{Fl}_{\mathbf{f}}\right)$ (Theorem 2.8).

Theorem 2.19 ([60, Thm. 5.2.3]) The restriction of the $\ell$-adic realization functor

$$
\rho_{\ell}: \operatorname{DM}\left(\mathrm{Fl}_{\mathbf{f}}\right) \rightarrow \mathrm{D}_{\mathrm{et}}\left(\mathrm{Fl}_{\mathbf{f}}, \mathbf{Q}_{\ell}\right)
$$

to the subcategory $\mathrm{DTM}\left(\mathrm{Fl}_{\mathbf{f}}\right)$ is conservative. Moreover, for $M \in \mathrm{DTM}\left(\mathrm{Fl}_{\mathbf{f}}\right)$ the following are equivalent: $a) M$ lies in $\operatorname{MTM}\left(\mathrm{Fl}_{\mathbf{f}}\right)$, and $\left.b\right) \rho_{\ell}(M)$ is a perverse sheaf.

The following corollary is useful in lifting results from the $\ell$-adic to the motivic setting.

Corollary 2.20 For each geometric point $f: \bar{s} \rightarrow S$, the composition of functors

$$
f^{*} \circ \rho_{\ell}: \operatorname{MTM}\left(\mathrm{Fl}_{\mathbf{f}}\right)^{\mathrm{c}} \rightarrow \operatorname{Perv}\left(\mathrm{Fl}_{\mathbf{f}, \bar{s}}, \mathbf{Q}_{\ell}\right)
$$

is well-defined, exact, conservative and faithful.

Proof Each object in $\mathrm{MTM}\left(\mathrm{Fl}_{\mathbf{f}}\right)^{\mathrm{c}}$ admits a Jordan-Hölder series (Theorem 2.8 (i)) whose simple constituents are the intersection motives $\mathrm{IC}_{w}(n)$ for $w \in W_{\mathbf{f}^{\prime}} \backslash W / W_{\mathbf{f}}$ and $n \in \mathbf{Z}$. Using the same method as in Proposition 2.15 we deduce that these are mapped under $\rho:=f^{*} \circ \rho_{\ell}$ to the corresponding $\ell$-adic intersection complex on $\mathrm{Fl}_{\mathbf{f}, \bar{s}}$. Since the subcategory $\operatorname{Perv}\left(\mathrm{Fl}_{\mathbf{f}, \bar{s}}, \mathbf{Q}_{\ell}\right) \subset \mathrm{D}_{\mathrm{et}}\left(\mathrm{Fl}_{\mathbf{f}}, \mathbf{Q}_{\ell}\right)$ is closed under extensions, it follows that $f^{*} \circ \rho_{\ell}$ is well-defined.

Being the restriction of an exact functor between triangulated categories, $\rho$ is exact as well. For the conservativity of $\rho$ it is therefore enough to show that the simple objects, namely the $\mathrm{IC}_{w}(n)$ are not mapped to 0 , which holds true by the above.

Being an exact conservative functor between abelian categories, $\rho$ is also faithful: If a morphism $p: A \rightarrow B$ maps to 0 under $\rho$, then $\operatorname{ker} \rho(p)=\rho(\operatorname{ker} p)=A$ by exactness. Hence, the natural map ker $p \rightarrow A$ is mapped to an isomorphism, and therefore is an isomorphism by conservativity. This shows $p=0$.

We can now prove the Kazhdan-Lusztig parity vanishing for intersection motives. Recall that for each class $w \in W_{\mathbf{f}^{\prime}} \backslash W / W_{\mathbf{f}}$ the relative dimension of $\mathrm{Fl}^{w} \rightarrow S$ is given by $l(w) \in \mathbf{Z}_{\geq 0}$ where $l=l\left(\mathbf{f}^{\prime}, \mathbf{f}\right)$ denotes the length function as in Sect. 2.1. Let $e_{w}: S=\{w\} \rightarrow \mathrm{Fl}_{\mathbf{f}}$ be the canonical inclusion. 
Theorem 2.21 For each $v, w \in W_{\mathbf{f}^{\prime}} \backslash W / W_{\mathbf{f}}, n \in \mathbf{Z}$, one has

$$
\mathrm{H}^{i}\left(e_{v}^{*} \mathrm{IC}_{w}(n)\right)=0 \quad \text { whenever } i \not \equiv l(w) \quad \bmod 2,
$$

where $\mathrm{H}^{i}$ denotes the truncation with respect to the classical motivic $t$-structure (cf. [60, Rem. 3.2.7]) which agrees on $S$ also with the perverse motivic t-structure (Theorem 2.8).

Proof We may assume $n=0$. By Corollary 2.20, it is enough to show that, for $S$ being the spectrum of a separably closed field, the $\ell$-adic intersection complex $\mathrm{IC}_{w, \ell}$ on $\mathrm{Fl}_{\mathbf{f}}^{\leq w}$ satisfies the parity vanishing (2.22) where $\mathrm{H}^{i}$ denotes the classical cohomology functor. This case is certainly well-known; we recall the part of the argument where we did not find a reference for the reader's convenience.

Reduction to the case $\mathbf{f}^{\prime}=\mathbf{f}=\mathbf{a}$. By refining the orbit stratification on $\mathrm{Fl}_{\mathbf{f}}$ we may assume that $\mathbf{f}^{\prime}=\mathbf{a}$ is the standard alcove. Now consider the projection $\pi: \mathrm{Fl}_{\mathbf{a}} \rightarrow \mathrm{Fl}_{\mathbf{f}}$ which is a smooth surjective map of relative dimension $d:=\operatorname{dim}\left(\mathcal{P}_{\mathbf{f}} / \mathcal{P}_{\mathbf{a}}\right)$ by $[60$, Prop. 4.3.13]. The preimage $\pi^{-1}\left(\mathrm{Fl}_{\mathbf{f}}^{\leq w}\right)$ is a Schubert scheme in $\mathrm{Fl}_{\mathbf{a}}$, and it follows from e.g. [60, Lem. 4.3 .7 (iii)] that

$$
\pi^{-1}\left(\mathrm{Fl}_{\mathbf{f}}^{\leq w}\right)=\mathrm{Fl}_{\mathbf{a}}^{\leq w_{\max }}
$$

where $w_{\max }$ is the unique representative of right maximal length with respect to $l_{\mathbf{a}}:=l(\mathbf{a}, \mathbf{a})$ in $w \cdot W_{\mathbf{f}}$. Its length is $l_{\mathbf{a}}\left(w_{\max }\right)=\operatorname{dim}\left(\mathrm{Fl}_{\mathbf{a}}^{\leq w_{\max }}\right)=l(w)+d$ by loc. cit.. As taking intermediate extensions commutes with smooth pullback, we have $\pi^{*}[d] \mathrm{IC}_{\ell, w}=\mathrm{IC}_{\ell, w_{\max }}$. Taking the cohomological shift into account and using the conservativity of pullback of surjective maps, we see that it is enough to prove (2.22) in the case $\mathbf{f}^{\prime}=\mathbf{f}=\mathbf{a}$.

Prooffor $\mathbf{f}^{\prime}=\mathbf{f}=\mathbf{a}$. Here we refer to the classical sources [26, A.7], [34] and [42].

\section{The convolution product}

In this section, we will discuss the tensor structure on the category $\operatorname{DM}\left(\mathcal{P}_{\mathbf{f}} \backslash L G / \mathcal{P}_{\mathbf{f}}\right)$ given by convolution. We start with the definition and basic properties in Sect. 3.1. In Sect. 3.2, we show that the convolution product preserves stratified Tate motives.

\subsection{Definition and associativity}

Definition 3.1 Let $\mathbf{f}^{\prime}, \mathbf{f}, \mathbf{f}^{\prime \prime}$ be three facets in the closure of the standard alcove, see Sect. 2.1. The convolution product is the functor

$$
\star: \operatorname{DM}\left(\mathcal{P}_{\mathbf{f}^{\prime}} \backslash L G / \mathcal{P}_{\mathbf{f}}\right) \times \operatorname{DM}\left(\mathcal{P}_{\mathbf{f}} \backslash L G / \mathcal{P}_{\mathbf{f}^{\prime \prime}}\right) \longrightarrow \operatorname{DM}\left(\mathcal{P}_{\mathbf{f}^{\prime}} \backslash L G / \mathcal{P}_{\mathbf{f}^{\prime \prime}}\right)
$$

defined by $\left(M_{1}, M_{2}\right) \mapsto M_{1} \star M_{2}:=m ! p^{!}\left(M_{1} \otimes M_{2}\right)$. Here the maps

$$
\mathcal{P}_{\mathbf{f}^{\prime}} \backslash L G / \mathcal{P}_{\mathbf{f}} \times \mathcal{P}_{\mathbf{f}} \backslash L G / \mathcal{P}_{\mathbf{f}^{\prime \prime}} \stackrel{p}{\longleftarrow} \mathcal{P}_{\mathbf{f}^{\prime}} \backslash L G \times \mathcal{P}_{\mathbf{f}} L G / \mathcal{P}_{\mathbf{f}^{\prime \prime}} \stackrel{m}{\longrightarrow} \mathcal{P}_{\mathbf{f}^{\prime}} \backslash L G / \mathcal{P}_{\mathbf{f}^{\prime \prime}}
$$


are the natural maps of prestacks induced by the identity on $L G \times L G$ (for $p$ ) and the multiplication $L G \times L G \rightarrow L G$ (for $m$ ).

Remark 3.2 (i) The left adjoint $m$ ! of the functor $m$ ! or, equivalently the left adjoint of the !-pullback along the ind-proper map of ind-schemes $\left(L G \times{ }^{\mathcal{P}_{\mathbf{f}}} L G / \mathcal{P}_{\mathbf{f}^{\prime \prime}}\right)^{\text {et }} \rightarrow$ $\left(L G / \mathcal{P}_{\mathbf{f}^{\prime \prime}}\right)^{\text {et }}=\mathrm{Fl}_{\mathbf{f}^{\prime \prime}}$ exists by [60, Lem. 2.2.9, Prop. 2.3.3].

(ii) The exterior product $M_{1} \otimes M_{2} \in \operatorname{DM}\left(\mathcal{P}_{\mathbf{f}^{\prime}} \backslash L G / \mathcal{P}_{\mathbf{f}} \times \mathcal{P}_{\mathbf{f}} \backslash L G / \mathcal{P}_{\mathbf{f}^{\prime \prime}}\right)$ exists by virtue of the construction in [60, Prop. 2.4.4], which gives a functor

$$
\otimes: \operatorname{DM}\left(\mathcal{P}_{\mathbf{f}^{\prime}} \backslash \mathrm{Fl}_{\mathbf{f}}\right) \otimes \operatorname{DM}\left(\mathcal{P}_{\mathbf{f}} \backslash \mathrm{Fl}_{\mathbf{f}^{\prime \prime}}\right) \rightarrow \operatorname{DM}\left(\mathcal{P}_{\mathbf{f}^{\prime}} \backslash \mathrm{Fl}_{\mathbf{f}} \times \mathcal{P}_{\mathbf{f}} \backslash \mathrm{Fl}_{\mathbf{f}^{\prime \prime}}\right)
$$

using the descent equivalence $\operatorname{DM}\left(\mathcal{P}_{\mathbf{f}^{\prime}} \backslash L G / \mathcal{P}_{\mathbf{f}}\right)=\operatorname{DM}\left(\mathcal{P}_{\mathbf{f}^{\prime}} \backslash \mathrm{Fl}_{\mathbf{f}}\right)$.

For clarity, we will momentarily denote the functor in (3.3) by $\otimes^{\mathrm{R}}$ and the convolution product stemming from this choice by $\star^{\mathrm{R}}$. Alternatively, we may consider

$$
\otimes^{\mathrm{L}}: \operatorname{DM}\left(\mathrm{Fl}_{\mathbf{f}^{\prime}}^{\mathrm{op}} / \mathcal{P}_{\mathbf{f}}\right) \otimes \operatorname{DM}\left(\mathrm{Fl}_{\mathbf{f}}^{\mathrm{op}} / \mathcal{P}_{\mathbf{f}^{\prime \prime}}\right) \rightarrow \operatorname{DM}\left(\mathrm{Fl}_{\mathbf{f}^{\prime}}^{\mathrm{op}} / \mathcal{P}_{\mathbf{f}} \times \mathrm{Fl}_{\mathbf{f}}^{\mathrm{op}} / \mathcal{P}_{\mathbf{f}^{\prime \prime}}\right)
$$

where $\mathrm{Fl}_{\mathbf{f}^{\prime}}^{\text {op }}=\left(\mathcal{P}_{\mathbf{f}^{\prime}} \backslash L G\right)^{\text {et }}$ and likewise for $\mathrm{Fl}_{\mathbf{f}}^{\text {op }}$. The resulting convolution product functor is denoted by $\star^{\mathrm{L}}$.

Proposition 3.4 On the level of the homotopy categories, the two functors $\star^{\mathrm{R}}$ and $\star^{\mathrm{L}}$ are naturally isomorphic, i.e., one has $\star^{\mathrm{R}} \cong \star^{\mathrm{L}}$ as functors

$$
\operatorname{Ho}\left(\operatorname{DM}\left(\mathcal{P}_{\mathbf{f}^{\prime}} \backslash L G / \mathcal{P}_{\mathbf{f}}\right)\right) \times \operatorname{Ho}\left(\operatorname{DM}\left(\mathcal{P}_{\mathbf{f}} \backslash L G / \mathcal{P}_{\mathbf{f}^{\prime \prime}}\right)\right) \longrightarrow \operatorname{Ho}\left(\operatorname{DM}\left(\mathcal{P}_{\mathbf{f}^{\prime}} \backslash L G / \mathcal{P}_{\mathbf{f}^{\prime \prime}}\right)\right)
$$

Proof Fix $v \leq w$ in $W_{\mathbf{f}^{\prime}} \backslash W / W_{\mathbf{f}}$ and consider the diagram

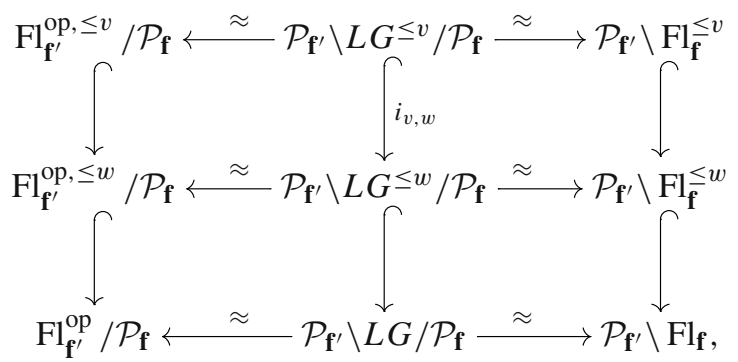

where $L G^{\leq w}=\mathcal{P}_{\mathbf{f}^{\prime}} w \mathcal{P}_{\mathbf{f}}$ denotes the scheme-theoretic image of $\mathcal{P}_{\mathbf{f}^{\prime}} \times \mathcal{P}_{\mathbf{f}} \rightarrow L G$, $\left(p, p^{\prime}\right) \mapsto p \cdot \dot{w} \cdot p^{\prime}$ where $\dot{w} \in L G(S)$ is any representative of $w$, and likewise for $L G^{\leq v}$. Note that this agrees with the preimage of $\mathrm{Fl}_{\mathbf{f}}^{\leq w}$ under the quotient map $L G \rightarrow \mathrm{Fl}_{\mathbf{f}}$, resp. the preimage of $\mathrm{Fl}_{\mathbf{f}^{\prime}}^{\mathrm{op}, \leq w}$ under $L G \rightarrow \mathrm{Fl}_{\mathbf{f}^{\prime}}^{\text {op }}$. The labels $\approx$ at the horizontal arrows indicate maps of prestacks which become equivalences after étale sheafification and therefore descent equivalences upon applying DM (Theorem 2.6).

By Corollary A.15, we have an exterior product, denoted by $\otimes$, for motives on placid prestacks such as the top middle term. Under the descent equivalence, it is compatible with the exterior product $\nabla^{\mathrm{L}}$ for motives on prestacks of the form as in 
the top left term, and similarly with the top right term. Of course, the same applies for the middle row as well. Moreover, these identifications are compatible with the pushforwards along the maps (induced by the closed embeddings $i_{v, w}$ ) between the top and middle row, i.e., there is a natural equivalence

$$
\alpha_{v, w}: \bigotimes^{w} \circ\left(i_{v, w}\right)_{*} \cong\left(i_{v, w}\right)_{*} \circ \bigotimes^{v}
$$

where $\nabla^{w}$ stands for an exterior product on terms as in the middle row of the diagram, and likewise for $\otimes^{v}$.

For yet another $u \leq v$ in $W_{\mathbf{f}^{\prime}} \backslash W / W_{\mathbf{f}}$, this equivalence and the one for $i_{u, v}$ and $i_{u, w}$ are compatible.

We obtain that the equivalence of $\infty$-categories

$$
\operatorname{DM}\left(\mathrm{Fl}_{\mathbf{f}^{\prime}}^{\mathrm{op}} / \mathcal{P}_{\mathbf{f}}\right) \cong \operatorname{DM}\left(\mathcal{P}_{\mathbf{f}^{\prime}} \backslash \mathrm{Fl}_{\mathbf{f}}\right)
$$

is compatible with exterior products ( $\otimes^{\mathrm{L}}$ and $\otimes^{\mathrm{R}}$, respectively), provided that we pass to the homotopy category and restrict to objects which are supported on some $\mathrm{Fl} \leq w$, op, resp. $\mathrm{Fl}^{\leq w}$. In particular, this is true for compact objects. We may drop this compactness condition, since the homotopy category of a compactly generated category, such as the categories DM on the above prestacks, is again compactly generated by [49, Rem. 1.4.4.3], and since the exterior product preserves filtered (homotopy) colimits separately in both variables.

Remark 3.6 The point of passing to the homotopy categories Ho(DM) is that these are ordinary categories, as opposed to $\infty$-categories DM. For this reason, it is enough to check the compatibility of $\alpha_{v, w}$ for two composable maps, as opposed to verifying higher coherences. We do not expect this loss of information to be necessary though: a more full-fledged approach would be to establish that $\mathrm{DM}^{!}$is a symmetric lax monoidal functor on the $\infty$-category of ind-placid prestacks, such as $\mathcal{P}_{\mathbf{f}}^{\prime} \backslash L G / \mathcal{P}_{\mathbf{f}}$.

Hereafter, we will write $\star$ for $\star^{R}$ above. Since our main interest in this paper lies in the convolution product on the abelian (in particular ordinary) category $\operatorname{MTM}\left(L^{+} G \backslash L G / L^{+} G\right)$, Proposition 3.4 shows that there is no ambiguity in the definition of the convolution product on this category.

As is well-known, the associativity of the convolution product is a consequence of the base-change formula:

Lemma 3.7 For $A \in \operatorname{DM}\left(\mathcal{P}_{\mathbf{f}^{\prime}} \backslash L G / \mathcal{P}_{\mathbf{f}}\right), \quad B \in \operatorname{DM}\left(\mathcal{P}_{\mathbf{f}} \backslash L G / \mathcal{P}_{\mathbf{f}}\right)$ and $C \in$ $\operatorname{DM}\left(\mathcal{P}_{\mathbf{f}} \backslash L G / \mathcal{P}_{\mathbf{f}^{\prime \prime}}\right)$, there is a natural equivalence

$$
(A \star B) \star C \cong A \star(B \star C)
$$


Proof For brevity, write $L:=L G$ throughout the proof. By construction in [60, Prop. 2.4.4] (and the associativity of the exterior product for motives on schemes in $\mathrm{Sch}_{S}^{\mathrm{ft}}$ ), the exterior product for three motives on the three prestacks in the lower left entry of the diagram below admits an associativity isomorphism $(A \otimes B) \otimes C \cong$ $A \otimes(B \otimes C)$. Up to the exterior product in the definition of $(A \star B) \star C$, this convolution is computed as $m ! p^{!}(m \times \mathrm{id}) !(p \times \mathrm{id}) !$

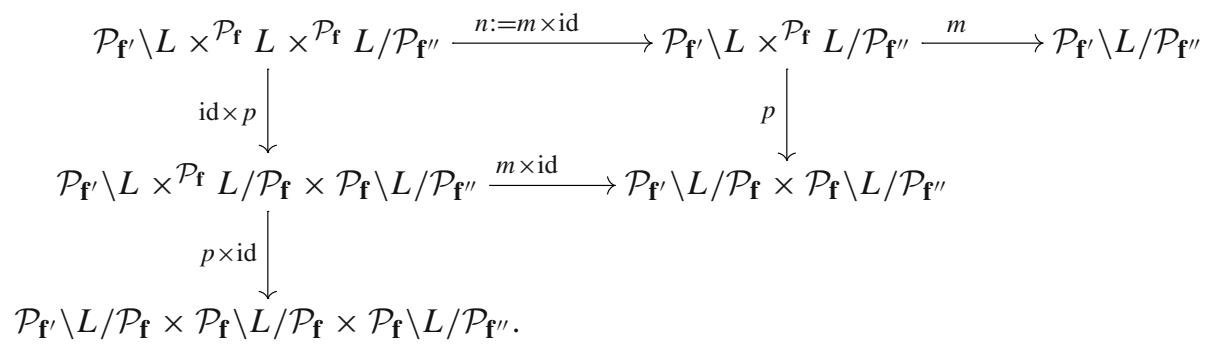

The square in the above diagram is (homotopy) cartesian. Moreover, the map $m$ is ind-proper, so that proper base change [60, Prop. 2.3.3] yields an equivalence

$$
p^{!}(m \times \mathrm{id}) ! \stackrel{\cong}{\longrightarrow} n_{!}(\mathrm{id} \times p) !
$$

Thus, the convolution $(A \star B) \star C$ can be computed by pullback and pushforward along the pictured composite correspondence. Considering instead the composition of the correspondences computing $A \star(B \star C)$, we obtain the same composition, which yields a zig-zag of equivalences.

\subsubsection{Reformulation in terms of schemes}

We now spell out the above definition in terms of ordinary schemes as opposed to prestacks. This relates to the classical definition of the convolution product as in [57, $\S 2$, and is used to show that the convolution product preserves Tate motives (Theorem 3.17 below).

Definition 3.9 Let $\mathbf{f}^{\prime}, \mathbf{f}, \mathbf{f}^{\prime \prime}$ be facets as in Definition 3.1. We define

$$
\mathrm{Fl}_{\mathbf{f}} \widetilde{\sim} \mathrm{Fl}_{\mathbf{f}^{\prime \prime}} \stackrel{\text { def }}{=}\left(L G \times{ }^{\mathcal{P}_{\mathbf{f}}} L G / \mathcal{P}_{\mathbf{f}^{\prime \prime}}\right)^{\mathrm{et}}
$$

which is an ind-proper $S$-ind-scheme. Consider the following commutative diagram of prestacks: 


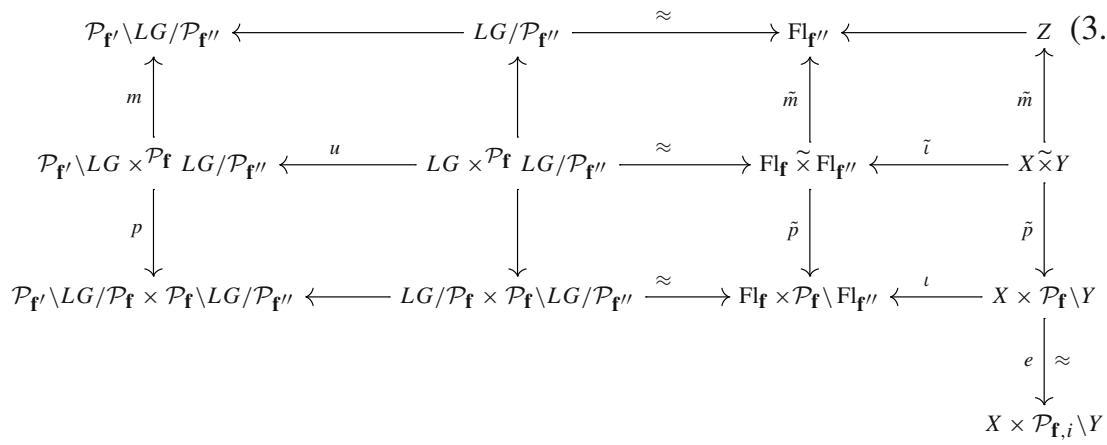

The left-hand horizontal maps such as $u$ are the natural quotient maps. By [60, Lem. 2.2.7], the !-pullback along such a map can be regarded as forgetting the $\mathcal{P}_{\mathbf{f}^{\prime}}$ action on some motive. According to [60, Thm. 2.2.16], the horizontal maps labelled “ $\approx$ " induce descent type equivalences after applying $\mathrm{DM}^{!}$. We will use similar equivalences without further comment; for example we identify motives on the double quotient $\mathcal{P}_{\mathbf{f}^{\prime}} \backslash L G / \mathcal{P}_{\mathbf{f}}$ with those on $\mathcal{P}_{\mathbf{f}^{\prime}} \backslash \mathrm{Fl}_{\mathbf{f}}$. The terms in the right hand column will be explained further below.

For $A \in \operatorname{DM}\left(\mathcal{P}_{\mathbf{f}^{\prime}} \backslash \mathrm{Fl}_{\mathbf{f}}\right), B \in \operatorname{DM}\left(\mathcal{P}_{\mathbf{f}} \backslash \mathrm{Fl}_{\mathbf{f}^{\prime \prime}}\right)$, the twisted box product is defined as

$$
A \widetilde{\otimes} B \stackrel{\text { def }}{=} u^{!} p^{!}(A \otimes B) \in \mathrm{DM}\left(\mathrm{Fl}_{\mathbf{f}} \widetilde{\times} \mathrm{Fl}_{\mathbf{f}^{\prime \prime}}\right) .
$$

Let $\tilde{m}: \mathrm{Fl}_{\mathbf{f}} \widetilde{\times} \mathrm{Fl}_{\mathbf{f}^{\prime \prime}} \rightarrow \mathrm{Fl}_{\mathbf{f}^{\prime \prime}}$ be the map of ind-schemes induced by multiplication, i.e., the map $m$ above is the non-sheafified version obtained by passing to the left- $\mathcal{P}_{\mathbf{f}^{\prime}}$ quotients. By virtue of the following lemma, we will denote $\tilde{m}$ simply by $m$ (it will be clear from the context which version we mean).

Lemma 3.11 For $A \in \operatorname{DM}\left(\mathcal{P}_{\mathbf{f}^{\prime}} \backslash \mathrm{Fl}_{\mathbf{f}}\right), B \in \operatorname{DM}\left(\mathcal{P}_{\mathbf{f}} \backslash \mathrm{Fl}_{\mathbf{f}^{\prime \prime}}\right)$, the object $\tilde{m}_{!}(A \widetilde{\nabla} B) \in$ $\operatorname{DM}\left(\mathrm{Fl}_{\mathbf{f}^{\prime \prime}}\right)$ is the non-equivariant object underlying $A \star B=m !(p !(A \otimes B))$.

Proof We only need to show that the functor $m$ ! commutes with the forgetful map to its non-equivariant version $\tilde{m}_{!}$. This is precisely the characterization of $m$ ! in [60, Lem. 2.2.9], see also Remark 3.2 (i) for its existence.

Both functors, $-\star-$ and $-\widetilde{\nabla}$ - preserve colimits separately in each variable. They therefore factor over the Lurie tensor product $\operatorname{DM}\left(\mathcal{P}_{\mathbf{f}^{\prime}} \backslash L G / \mathcal{P}_{\mathbf{f}}\right) \otimes \operatorname{DM}\left(\mathcal{P}_{\mathbf{f}} \backslash L G / \mathcal{P}_{\mathbf{f}^{\prime \prime}}\right)$. Since categories of motives are compactly generated [60, Lem. 2.3.6], the functors are therefore determined by their values on compact objects. Suppose then that $A$ and $B$ are compact objects, so they are supported on closed, finite type subschemes $X \subset \mathrm{Fl}_{\mathbf{f}}$, $Y \subset \mathrm{Fl}_{\mathbf{f}^{\prime \prime}}$ which are finite unions of Schubert schemes (these are the objects in the right vertical column in (3.10)). The right-most vertical maps in the diagram, such as $\iota$ are induced by the closed embeddings of these subschemes. In this case, $A \widetilde{\nabla} B$ admits the following description:

Let $\mathcal{P}_{\mathbf{f}}=\lim _{i \geq 0} \mathcal{P}_{\mathbf{f}, i}$ as in [60, Lem. 4.2.7] and denote $\mathcal{U}_{\mathbf{f}, i}:=\operatorname{ker}\left(\mathcal{P}_{\mathbf{f}} \rightarrow \mathcal{P}_{\mathbf{f}, i}\right)$. We let $X_{i} \subset \mathrm{Fl}_{\mathbf{f}, i}:=\left(L G / \mathcal{U}_{\mathbf{f}, i}\right)^{\text {et }}$ (resp. $X_{\infty} \subset L G$ ) be the finite type (resp. nonfinite type) $S$-scheme defined by the preimage of $X$ under the canonical projection 
$\mathrm{Fl}_{\mathbf{f}, i} \rightarrow \mathrm{Fl}_{\mathbf{f}}$ (resp. $L G \rightarrow \mathrm{Fl}_{\mathbf{f}}$ ). The left- $\mathcal{P}_{\mathbf{f}}$-action on $Y$ factors through some $\mathcal{P}_{\mathbf{f}, i}$ for $i \gg 0$. We write $X \widetilde{\times} Y:=X_{\infty} \times{ }^{\mathcal{P}_{\mathbf{f}}} Y$, which is equivalent to $X_{i} \times{ }^{\mathcal{P}_{\mathbf{f}, i}} Y$ since $\left(X_{\infty} / \mathcal{P}_{\mathbf{f}}\right)^{\text {et }}=X$ and $\left(X_{\infty} / \mathcal{U}_{\mathbf{f}, i}\right)^{\text {et }}=X_{i}$. The vertical map $e$ labelled $\approx$ in the above diagram yields an equivalence upon applying $\mathrm{DM}^{!}$(this stems from $\mathbf{A}^{1}$-invariance, using that $\mathcal{U}_{\mathbf{f}, i}$ is split pro-unipotent, see [60, Prop. 2.2.11]). In particular, we can regard $B \in \operatorname{DM}\left(\mathcal{P}_{\mathbf{f}} \backslash Y\right)$ as an object in $\operatorname{DM}\left(\mathcal{P}_{\mathbf{f}, i} \backslash Y\right)$. By the support setup, we can write $A \otimes B$ as $\iota !\left(A_{0} \otimes B_{0}\right)$ with $A_{0}=\iota^{*} A$ etc., so that

$$
A \widetilde{\otimes} B=(e \circ \tilde{p})^{!}(A \otimes B)=\tilde{\iota} !\left((e \circ \tilde{p})^{!} A_{0} \otimes B_{0}\right) .
$$

Note that the schemes $X, Y, \mathcal{P}_{\mathbf{f}, i}$ and $Z$ intervening in the correspondence $X \times$ $\mathcal{P}_{\mathbf{f}, i} \backslash Y \stackrel{e \circ p}{\leftarrow} X \tilde{\times} Y \stackrel{\tilde{m}}{\rightarrow} Z$, are of finite type over $S$ (unlike the remaining terms in the diagram). We also see that the above definition of $\widetilde{\nabla}$ agrees with the definition of $\widetilde{\nabla}$ used for example in [57, Lem. 2.20, Rmk. 2.21].

Finally, writing $Z:=\tilde{m}(X \tilde{\times} Y)$ (scheme-theoretic image, again a finite type $S$ scheme), $A \star B$ has as its underlying non-equiviarant object $\tilde{m}_{!}(A \widetilde{\nabla} B)$, which is, by proper base change, the !-pushforward along $Z \subset \mathrm{Fl}_{\mathbf{f}}$, of $\tilde{m}_{!}(e \circ \tilde{p}) !\left(A_{0} \otimes B_{0}\right)$.

Lemma 3.12 (i) Let $X, Y$ be as above. Then $1_{X} \widetilde{\otimes} 1_{Y}=1_{X} \tilde{\times} Y \in \operatorname{DM}(X \tilde{\times} Y)$.

(ii) If $\xi: X \rightarrow X^{\prime}$ (resp. $v: Y \rightarrow Y^{\prime}$ ) is an inclusion of finite type $\mathcal{P}_{\mathbf{f}}^{\prime}$-equivariant (resp. $\mathcal{P}_{\mathbf{f}}$-equivariant) subschemes of $\mathrm{Fl}_{\mathbf{f}}$ (resp. of $\mathrm{Fl}_{\mathbf{f}}$ ) then

$$
(\xi \widetilde{\times} v)_{!}(A \widetilde{\otimes} B)=\xi ! A \widetilde{\otimes} v_{!} B
$$

Proof The maps $X_{i} \times{ }^{\mathcal{P}_{\mathbf{f}, i}} Y \rightarrow X \times \mathcal{P}_{\mathbf{f}, i} \backslash Y \leftarrow X \times Y$ induce forgetful maps

$$
\operatorname{DM}\left(X_{i} \times{ }^{\mathcal{P}_{\mathbf{f}, i}} Y\right) \leftarrow \operatorname{DM}\left(X \times \mathcal{P}_{\mathbf{f}, i} \backslash Y\right) \rightarrow \operatorname{DM}(X \times Y),
$$

under which $1_{X} \otimes 1_{Y}=1_{X \times Y}$ corresponds to $1_{X} \tilde{\times} Y$ under !-pullback. For the second statement note that the map $r:=e \circ \tilde{p}$ in (3.10) is a $\mathcal{P}_{\mathbf{f}, i}$-torsor, in particular a smooth map (of finite type $S$-schemes). Therefore $r^{!}$commutes with the exterior product and with the !-pushforward along the embeddings $X \times \mathcal{P}_{\mathbf{f}, i} Y \rightarrow X^{\prime} \times \mathcal{P}_{\mathbf{f}, i} Y^{\prime}$ and $\xi \tilde{\times} v: X \tilde{\times} Y \rightarrow X^{\prime} \tilde{\times} Y^{\prime}$.

\subsubsection{Compatibility with the $\ell$-adic realization}

We denote by $\mathrm{D}_{\mathrm{ct}, \mathcal{P}_{\mathbf{f}}}^{\mathrm{b}}\left(\mathrm{Fl}_{\mathbf{f}^{\prime \prime}}, \mathbf{Q}_{\ell}\right)$ the category of $\mathcal{P}_{\mathbf{f}}$-equivariant $\ell$-adic sheaves on $\mathrm{Fl}_{\mathbf{f}^{\prime \prime}}$. In [52] (see also [54, §10.2] and [57, §2]), the convolution product for $\ell$-adic sheaves

$$
\star_{\ell}: \mathrm{D}_{\mathrm{ct}}^{\mathrm{b}}\left(\mathrm{Fl}_{\mathbf{f}}, \mathbf{Q}_{\ell}\right) \times \mathrm{D}_{\mathrm{ct}, \mathcal{P}_{\mathbf{f}}}^{\mathrm{b}}\left(\mathrm{Fl}_{\mathbf{f}^{\prime \prime}}, \mathbf{Q}_{\ell}\right) \rightarrow \mathrm{D}_{\mathrm{ct}}^{\mathrm{b}}\left(\mathrm{Fl}_{\mathbf{f}^{\prime \prime}}, \mathbf{Q}_{\ell}\right)
$$

is defined as follows: consider the diagram

$$
\mathrm{Fl}_{\mathbf{f}} \times \mathrm{Fl}_{\mathbf{f}^{\prime \prime}} \stackrel{p}{\longleftarrow} L G \times \mathrm{Fl}_{\mathbf{f}^{\prime \prime}} \stackrel{q}{\longrightarrow} \mathrm{Fl}_{\mathbf{f}} \widetilde{\times} \mathrm{Fl}_{\mathbf{f}^{\prime \prime}}=\left(L G \times{ }^{\mathcal{P}}{ }_{\mathbf{f}} L G / \mathcal{P}_{\mathbf{f}^{\prime \prime}}\right)^{\text {et }} \stackrel{m}{\longrightarrow} \mathrm{Fl}_{\mathbf{f}^{\prime \prime}}
$$


Then $A_{1} \star_{\ell} A_{2}:=m_{*}\left(A_{1} \widetilde{\otimes}_{\ell} A_{2}\right)$, where $A_{1} \widetilde{\otimes}_{\ell} A_{2}$ is the unique object in $\mathrm{D}_{\mathrm{ct}}^{\mathrm{b}}\left(\mathrm{Fl}_{\mathbf{f}} \widetilde{\times} \mathrm{Fl}_{\mathbf{f}^{\prime \prime}}, \mathbf{Q}_{\ell}\right)$ such that $p^{*}\left(A_{1} \otimes_{\ell} A_{2}\right)=q^{*}\left(A_{1} \widetilde{\otimes}_{\ell} A_{2}\right)$, using the $\mathcal{P}_{\mathbf{f}^{-}}$ equivariance of $A_{2}$.

Proposition 3.14 Under the $\ell$-adic realization functor $\rho_{\ell}$ (cf. [60, Thm. 2.3.7]) the convolution product corresponds to the convolution product $\star_{\ell}$ considered in the context of the $\ell$-adic Satake equivalence, i.e., there is a natural isomorphism

$$
\rho_{\ell}(A \star B) \cong \rho_{\ell}(A) \star \ell \rho_{\ell}(B)
$$

for $A \in \operatorname{DM}\left(L G / \mathcal{P}_{\mathbf{f}}\right)^{\mathrm{c}}$ and $B \in \operatorname{DM}\left(\mathcal{P}_{\mathbf{f}} \backslash L G / \mathcal{P}_{\mathbf{f}^{\prime \prime}}\right)^{\mathrm{c}}$.

Proof The twisted box product $\widetilde{\nabla}$ for motives (Definition 3.9) is formed using descent along !-pullbacks. We need to compare its $\ell$-adic realization with $\widetilde{\otimes}_{\ell}$, formed using *-pullbacks.

Since the objects $A, B$ are compact, hence supported on $S$-finite type closed subschemes $X \subset \mathrm{Fl}_{\mathbf{f}}, Y \subset \mathrm{Fl}_{\mathbf{f}^{\prime \prime}}$, we can replace the maps $p, q$ in (3.13) by the diagram of $\mathcal{P}_{\mathbf{f}, i}$-torsors

$$
X \times Y \stackrel{p}{\longleftarrow} X_{i} \times Y \stackrel{q}{\longrightarrow} X \tilde{\times} Y
$$

for some $i \geq 0$ as in Sect. 3.1.1 above. Let $G:=\mathcal{P}_{\mathbf{f}, i}$ which is a smooth affine $S$-group scheme acting on $Z:=X_{i} \times Y$ either via the torsor $p$ or $q$. By definition, the category of $G$-equivariant $\ell$-adic sheaves on $Z$ is defined as

$$
\begin{aligned}
\mathrm{D}_{\mathrm{ct}, G}^{\mathrm{b}}\left(Z, \mathbf{Q}_{\ell}\right) & :=\lim \left(\left(\mathrm{D}_{\mathrm{ct}}^{\mathrm{b}}\right)^{*}\left(\operatorname{Bar}(Z, G), \mathbf{Q}_{\ell}\right)\right) \\
& :=\lim \left(\mathrm{D}_{\mathrm{ct}}^{\mathrm{b}}\left(Z, \mathbf{Q}_{\ell}\right) \underset{p^{*}}{\stackrel{a^{*}}{\rightrightarrows}} \mathrm{D}_{\mathrm{ct}}^{\mathrm{b}}\left(G \times Z, \mathbf{Q}_{\ell}\right) \underset{\rightarrow}{\rightarrow} \cdots\right),
\end{aligned}
$$

where we emphasize that the functors in this limit are the *-pullbacks along the maps in the bar complex. The motivic analogue of that category is $\operatorname{DM}^{*}(G \backslash Z)^{\mathrm{c}}:=$ $\lim \mathrm{DM}^{*}(\operatorname{Bar}(Z, G))^{\mathrm{c}}$, where again we use $*$-pullbacks to form the limit. (See also [60, Rem. 2.2.2, (iv)] for further discussion of the presheaf $\mathrm{DM}^{*}$.)

The vertices $(G)^{\times n} \times Z$ of the bar construction are separated $S$-schemes of finite type, and the action and projection maps in this diagram are smooth and affine, noting that $G \rightarrow S$ is so. We can therefore use the equivalence of $\mathrm{DM}^{*}$ with $\mathrm{DM}^{!}$applied to the smooth morphisms $p, q$, see Corollary A.8. Under this equivalence the functor $p^{!}\left(\right.$resp. $\left.q^{!}\right)$corresponds to $p^{*}$ (resp. $\left.q^{*}\right)$. Since $p, q$ have the same relative dimension $\operatorname{dim}(G / S)$, we can equivalently form $A \widetilde{\otimes} B$ using descent along $*$-pullbacks. Moreover, the map $m$ is ind-proper, so that $m_{*}=m$ !. We conclude using that $\rho_{\ell}$ is compatible with the six functors.

\subsubsection{Convolution product and change of base scheme}

Lemma 3.15 Let $f: T \rightarrow S$ be a map of schemes satisfying the assumptions in Notation 2.1. Then, for $M_{1} \in \operatorname{DM}\left(\mathcal{P}_{\mathbf{f}^{\prime}} \backslash L G / \mathcal{P}_{\mathbf{f}}\right)$ and $M_{2} \in \operatorname{DM}\left(\mathcal{P}_{\mathbf{f}^{\prime}} \backslash L G / \mathcal{P}_{\mathbf{f}}\right)$, there is a 
natural isomorphism

$$
f^{*}\left(M_{1} \star M_{2}\right) \cong\left(f^{*} M_{1}\right) \star\left(f^{*} M_{2}\right)
$$

Proof All functors involved in the definition of $\star$ are compatible with $f^{*}$. For $p^{!}$, this holds true since (the étale sheafification of) $p$ is a $\mathcal{P}_{\mathbf{f}}$-torsor, in particular pro-smooth.

\subsection{Preservation of Tate motives}

In this section, we show that the convolution product on partial affine flag varieties respects stratified Tate motives. A key point in this proof is the well-knwon distinguished triangle (3.21) below (cf. [41, App.]), which is a geometric incarnation of the following formula for the multiplication in the Iwahori-Hecke algebra over a finite field $\mathbf{F}_{q}$ given in [11, IV, §2, Ex. 24]:

$$
\phi_{s} \star \phi_{s}=(q-1) \cdot \phi_{s}+q \cdot \phi_{e},
$$

where $\phi_{s}$ is the characteristic function of the Iwahori double coset $\mathcal{B}\left(\mathbf{F}_{q}\right) s \mathcal{B}\left(\mathbf{F}_{q}\right)$ for a simple reflection $s$, and $\phi_{e}$ is the characteristic function of the base point. Here $\mathcal{B}:=\mathcal{P}_{\mathbf{a}}$ denotes the standard Iwahori subgroup associated with the choice of the alcove a.

Theorem 3.17 Let the base scheme $S$ be as in Notation 2.1. For any three facets $\mathbf{f}^{\prime}, \mathbf{f}, \mathbf{f}^{\prime \prime}$ contained in the closure of $\mathbf{a}$, the convolution product restricts to a functor

$$
\star: \operatorname{DTM}\left(\mathcal{P}_{\mathbf{f}^{\prime}} \backslash L G / \mathcal{P}_{\mathbf{f}}\right) \times \operatorname{DTM}\left(\mathcal{P}_{\mathbf{f}} \backslash L G / \mathcal{P}_{\mathbf{f}^{\prime \prime}}\right) \rightarrow \operatorname{DTM}\left(\mathcal{P}_{\mathbf{f}^{\prime}} \backslash L G / \mathcal{P}_{\mathbf{f}^{\prime \prime}}\right)
$$

In particular, taking $\mathbf{f}^{\prime}=\mathbf{f}=\mathbf{f}^{\prime \prime}=0$, there is a convolution product on $\operatorname{DTM}\left(L^{+} G \backslash L G / L^{+} G\right)$.

Let $A \in \operatorname{DTM}\left(\mathcal{P}_{\mathbf{f}^{\prime}} \backslash L G / \mathcal{P}_{\mathbf{f}}\right), B \in \operatorname{DTM}\left(\mathcal{P}_{\mathbf{f}} \backslash L G / \mathcal{P}_{\mathbf{f}^{\prime \prime}}\right)$ and denote $\mathrm{Fl}_{\mathbf{f}^{\prime \prime}}:=$ $\left(L G / \mathcal{P}_{\mathbf{f}^{\prime \prime}}\right)^{\text {et }}$. By [60, Def. 3.1.21], we have to show that the non-equivariant motive underlying $A \star B$ in $\mathrm{DM}\left(\mathrm{Fl}_{\mathbf{f}^{\prime \prime}}\right)$ is Tate. Further, we may assume that both objects $A, B$ are compact. By [60, Thm. 5.3.4], the motives $A$ and $B$ are constructed in finitely many steps from the generators

$$
\iota_{w, !} 1 \in \operatorname{DTM}\left(\mathcal{P}_{\mathbf{f}^{\prime}} \backslash L G / \mathcal{P}_{\mathbf{f}}\right),
$$

where $w \in W_{\mathbf{f}^{\prime}} \backslash W / W_{\mathbf{f}}$ and $\iota_{w}: \mathcal{P}_{\mathbf{f}^{\prime}} \backslash L G^{w} / \mathcal{P}_{\mathbf{f}} \rightarrow \mathcal{P}_{\mathbf{f}^{\prime}} \backslash L G / \mathcal{P}_{\mathbf{f}}$ denotes the inclusion of the stratum ${ }^{1} L G^{w}:=\mathcal{P}_{\mathbf{f}^{\prime}} \dot{w} \mathcal{P}_{\mathbf{f}}$ where $\dot{w} \in L G(S)$ is any representative of $w$. We have to show that the non-equivariant motive underlying $\iota_{w, !} 1 \star \iota_{w^{\prime}, !} 1$ is Tate for any $w \in W_{\mathbf{f}^{\prime}} \backslash W / W_{\mathbf{f}}, w^{\prime} \in W_{\mathbf{f}} \backslash W / W_{\mathbf{f}^{\prime \prime}}$, i.e., by Lemma 3.11 , that

$$
\tilde{m}_{!}\left(\iota_{w, !} 1 \widetilde{\nabla}_{\iota_{w^{\prime}, !}}\right) \in \operatorname{DTM}\left(\mathrm{Fl}_{\mathbf{f}^{\prime \prime}}\right) \text {. }
$$

\footnotetext{
${ }^{1}$ Formally, $L G^{w}$ is the scheme-theoretic image of the map $\mathcal{P}_{\mathbf{f}^{\prime}} \times \mathcal{P}_{\mathbf{f}} \rightarrow L G,(b, p) \mapsto b \cdot \dot{w} \cdot p$ which is well defined because the source is quasi-compact and the target is an ind-scheme.
} 
We show (3.18) in several steps starting with the following key case.

Proposition 3.19 If $\mathcal{P}_{\mathbf{f}^{\prime}}=\mathcal{P}_{\mathbf{f}}=\mathcal{P}_{\mathbf{f}^{\prime \prime}}=: \mathcal{B}$ is the standard Iwahori subgroup, then Theorem 3.17 holds.

Proof In this case, $W_{\mathbf{f}^{\prime}}=W_{\mathbf{f}}=W_{\mathbf{f}^{\prime \prime}}=1$, so that $w, w^{\prime} \in W$.

First case. Assume that $w=w^{\prime}=s$ is a simple reflection. Then there is an isomorphism

$$
\tau=(p, \tilde{m}): \mathrm{Fl}^{\leq s} \widetilde{\times} \mathrm{Fl}^{\leq s} \stackrel{\simeq}{\longrightarrow} \mathrm{Fl}^{\leq s} \times \mathrm{Fl}^{\leq s},
$$

where $p:(x, y) \mapsto x$ is the projection on the first factor, and $\tilde{m}:(x, y) \mapsto x \cdot y$ is the multiplication map, as above. Note that the image of $\tilde{m}$ lies inside $\mathrm{Fl}^{\leq s}$ by standard properties of Tits systems [11, Ch. IV, §2.1, (2)] which are applicable in view of $[12,5.2 .12]$ (see also the discussion in $[58, \S 1.1]$ ). Further, $\tau$ being a closed immersion (being a proper monomorphism) between integral schemes of the same dimension, it must be an isomorphism. Under this isomorphism, we have for the strata $\tau(\{e\} \tilde{\times}\{e\})=\{e\} \times\{e\}, \tau\left(\{e\} \widetilde{\times} \mathrm{Fl}^{s}\right)=\{e\} \times \mathrm{Fl}^{s}$ and $\tau\left(\mathrm{Fl}^{s} \widetilde{\times}\{e\}\right)=\Delta\left(\mathrm{Fl}^{s}\right)$, where $\Delta: \mathrm{Fl}^{\leq s} \rightarrow \mathrm{Fl}^{\leq s} \times \mathrm{Fl}^{\leq s}$ denotes the diagonal. We conclude that

$$
\tau\left(\mathrm{Fl}^{s} \widetilde{\times} \mathrm{Fl}^{s}\right)=\left(\mathrm{Fl}^{s} \times \mathrm{Fl}^{\leq s}\right) \backslash \Delta\left(\mathrm{Fl}^{s}\right) .
$$

Identifying $\mathrm{Fl}^{\leq s} \simeq \mathbf{P}^{1}$ such that $\{e\} \simeq\{\infty\}$ and $\mathrm{Fl}^{s} \simeq \mathbf{A}^{1}$, we get a commutative diagram of $S$-schemes

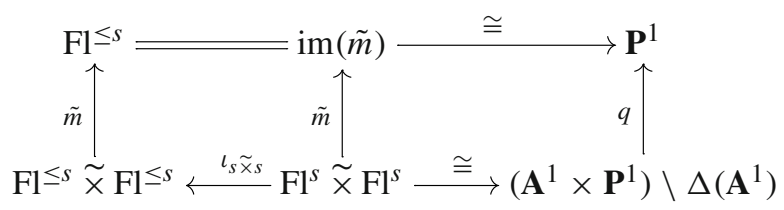

where $\iota_{s} \tilde{\times} s$ and $\iota_{s}$ are the inclusion of the open strata and $q$ is the projection onto the second factor. Writing $a:=\tilde{m} \circ \iota_{s} \tilde{\times} s$ and using Lemma 3.12 for $\iota_{s} \tilde{\times} s=\iota_{s} \widetilde{\times} \iota_{s}$, we have to prove

$$
\tilde{m}_{!}\left(\iota_{s, !} 1 \widetilde{\otimes} \iota_{s, !} 1\right)=\tilde{m}_{!}\left(\iota_{s} \widetilde{\times} \iota_{s}\right) ! 1=a_{!} 1 \in \mathrm{DTM}(\mathrm{Fl} \leq s),
$$

or equivalently that

$$
M:=q ! 1 \in \operatorname{DTM}\left(\mathbf{P}^{1}\right)
$$

where the Tateness of the motive is with respect to the stratification of $\mathbf{P}^{1} \simeq \mathrm{Fl}^{\leq s}$ by $\{\infty\} \sqcup \mathbf{A}^{1} \simeq \mathrm{Fl}^{e} \sqcup \mathrm{Fl}^{s}$. To check this, we use the localization sequence, noting that $q^{-1}(\{\infty\}) \simeq \mathbf{A}^{1}$ and $q^{-1}\left(\mathbf{A}^{1}\right) \simeq \mathbf{A}^{1} \times \mathbf{G}_{\mathrm{m}}$ which gives 
$\left(\iota_{\mathbf{A}^{1}}\right) ! \iota_{\mathbf{A}^{1}}^{*} M=\iota_{\mathbf{A}^{1}, !}(1(-1)[-2] \oplus 1[-1]) \longrightarrow M \longrightarrow \iota_{\infty, !} i_{\infty}^{*} M=\iota_{\infty !} 1(-1)[-2]$.

The outer terms are in $\operatorname{DTM}\left(\mathbf{P}^{1}\right)$, hence so is the middle. This finishes the first case.

Second case. Let $w, w^{\prime} \in W$, and assume $l\left(w w^{\prime}\right)=l(w)+l\left(w^{\prime}\right)$ for the Bruhat length. Then the composition of the open inclusion $\iota_{w} \tilde{\times} w^{\prime}: \mathrm{Fl}^{w} \widetilde{\times} \mathrm{Fl}^{w^{\prime}} \rightarrow$ $\mathrm{Fl} \leq w \widetilde{\times} \mathrm{Fl}^{\leq w^{\prime}}$ with the multiplication map $m$ is an isomorphism

$$
m \circ j_{w} \tilde{\times} w^{\prime}: \mathrm{Fl}^{w} \widetilde{\times} \mathrm{Fl}^{w^{\prime}} \stackrel{\simeq}{\longrightarrow} \mathrm{Fl}^{w w^{\prime}} .
$$

This implies that $\iota_{w, !} 1 \star \iota_{w^{\prime}, !} 1 \simeq \iota_{w w^{\prime}, !} 1 \in \mathrm{DTM}(\mathrm{Fl})$ is Tate and finishes the second case.

Third case. Let $w, w^{\prime} \in W$, and assume that $w^{\prime}=s$ is a simple reflection. If $l(w s)=l(w)+1$, then we conclude $\iota_{w, !} 1 \star l_{s, !} 1 \in \mathrm{DTM}(\mathrm{Fl})$ by the second case. If $l(w s)=l(w)-1$, we write $v:=w s$. Since $s^{2}=1$, we have $w=v s$, and by construction $l(v s)=l(v)+1$. In particular, $\iota_{w, !} 1 \simeq \iota_{v, !} 1 \star \iota_{s, !} 1$ by the second step. Applying $\iota_{v, !} 1 \star(-)$ to the localization sequence (3.21), we get a cofiber sequence

$$
\left(\iota_{w, !} 1(-1)[-2] \oplus \iota_{w, !} 1[-1]\right) \longrightarrow \iota_{v, !} 1 \star\left(\iota_{s, !} 1 \star \iota_{s, !} 1\right) \longrightarrow \iota_{v, !} 1(-1)[-2] \longrightarrow
$$

where $\iota_{v, !} 1 \star\left(\iota_{s, !} 1 \star l_{s, !} 1\right) \simeq\left(\iota_{v, !} 1 \star l_{s, !} 1\right) \star l_{s, !} 1 \simeq \iota_{w, !} 1 \star l_{s, !} 1$ by Lemma 3.7. Hence, $\iota_{w, !} 1 \star \iota_{s, !} 1$ is an extension of Tate motives on Fl, and thus defines an object in DTM(Fl). This finishes the third case.

General case. Let $w, w^{\prime} \in W$ be arbitrary. Fix a reduced expression $w^{\prime}=s_{1} \cdot \ldots \cdot s_{n}$ where $s_{i}$ are simple reflections and $n=l\left(w^{\prime}\right)$. By the second case, we have $\iota_{w^{\prime}, !} \simeq$ $\iota_{s_{1}, !} 1 \star \ldots \star l_{s_{n}, !} 1$ where we omit the parenthesis in view of Lemma 3.7. By repeated use of the third case, we conclude that $\iota_{w, !} 1 \star \iota_{w^{\prime}, !} 1 \in \mathrm{DTM}(\mathrm{Fl})$ is Tate. This finishes the general case, and the theorem follows.

Remark 3.24 If $k=\mathbf{F}_{q}$, (3.21) gets mapped by the $\ell$-adic realization to

$$
\iota_{s, !} \mathbf{Q}_{\ell}(-1)[-2] \oplus \iota_{s, !} \mathbf{Q}_{\ell}[-1] \rightarrow \iota_{s, !} \mathbf{Q}_{\ell} \star \iota_{s, !} \mathbf{Q}_{\ell} \rightarrow \iota_{\ell, !} \mathbf{Q}_{\ell}(-1)[-2]
$$

Taking the alternating trace of the geometric Frobenius, we obtain the identity (3.16) using the relation trace $\left(\right.$ Frob $\left.\mid \mathbf{Q}_{\ell}(-1)\right)=q$.

Remark 3.25 The method used in the proof of Theorem 3.17 works more generally for not necessarily split reductive groups $G$ defined over $k((\varpi))$ which are residually split, i.e., $\mathrm{Fl}^{s} \simeq \mathbf{A}_{k}^{1}$ whenever $s \in W$ is a simple reflection. However, we will not need these non-split cases in this manuscript.

Proposition 3.26 Theorem 3.17 holds in the case that $\mathcal{P}_{\mathbf{f}^{\prime}}=\mathcal{P}_{\mathbf{f}^{\prime \prime}}=\mathcal{B}$ is the standard Iwahori subgroup of $L G$, and any facet $\mathbf{f}$ in the closure of the standard alcove (so that $\mathcal{B}$ is a subgroup of $\left.\mathcal{P}_{\mathbf{f}}\right)$. 
Proof Let $\mathcal{P}:=\mathcal{P}_{\mathbf{f}}$. For any $w \in W / W_{\mathbf{f}}, w^{\prime} \in W_{\mathbf{f}} \backslash W$, consider the diagram

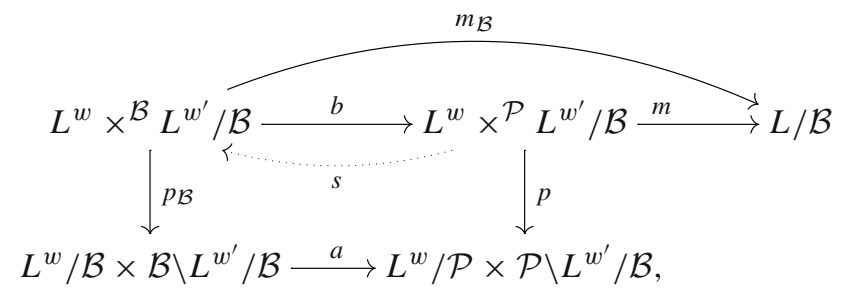

where $L^{w}:=L G^{w}=\mathcal{B} w \mathcal{P} \subset L G$ (resp. $L^{w^{\prime}}:=L G^{w^{\prime}}=\mathcal{P} w^{\prime} \mathcal{B} \subset L G$ ). То construct the map $s$ with $b \circ s=\mathrm{id}$, it suffices to construct a section to the composition of quotient maps

$$
L^{w} \times L^{w^{\prime}} \rightarrow L^{w} \times{ }^{\mathcal{B}} L^{w^{\prime}} \rightarrow L^{w} \times{ }^{\mathcal{P}} L^{w^{\prime}}
$$

which is equivariant for right $\mathcal{B}$-action on the second factor. It follows from [60, Prop. 4.3.9] that there exists a closed subscheme $U \subset \mathcal{B}$, a finite direct product of some affine root groups (depending on $w$ ), such that the multiplication $U \dot{w} \times \mathcal{P} \rightarrow$ $L^{w}$ is an isomorphism. Here $\dot{w} \in W$ is any representative of $w \in W / W_{\mathbf{f}}$. Thus, $L^{w} \times{ }^{\mathcal{P}} L^{w^{\prime}} \simeq U \dot{w} \times L^{w^{\prime}} \subset L^{w} \times L^{w^{\prime}}$ is the desired section.

In particular, the adjunction map $b_{!} b^{!} \rightarrow$ id has the section id $\simeq b_{!} s ! s^{!} b^{!} \rightarrow b_{!} b$, so that the motive $M:=p^{!}\left(\iota_{w, !} \otimes \iota_{w^{\prime}, !} 1\right) \in \operatorname{DM}\left(L^{w} \times{ }^{\mathcal{P}} L^{w^{\prime}} / \mathcal{B}\right)$ is a direct summand of $b_{!} b^{!} M$. Thus, the motive $m ! M=\iota_{w, !} 1 \star \iota_{w^{\prime}, !} 1$ is a direct summand of the motive

$$
m ! b ! b^{!} M \simeq(m \circ b) !(p \circ b)^{!}\left(\iota_{w, !} 1 \otimes \iota_{w^{\prime}, !} 1\right) \simeq m_{\mathcal{B}, !} p_{\mathcal{B}}\left(a^{!}\left(\iota_{w, !} 1 \otimes \iota_{w^{\prime}, !} 1\right)\right)
$$

The map $\mathrm{Fl}_{\mathbf{a}}^{w} \times \mathrm{Fl}_{\mathbf{a}}^{w^{\prime}} \rightarrow \mathrm{Fl}_{\mathbf{f}}^{w} \times \mathrm{Fl}_{\mathbf{a}}^{w^{\prime}}$ is stratified with respect to the stratification by $\mathcal{B} \times \mathcal{P}$-orbits. Hence $a^{!}\left(\iota_{w, !} 1 \otimes \iota_{w^{\prime}, !} 1\right)$ is stratified Tate. Therefore the motive $m_{!} b ! b^{!} M$ is stratified Tate by Proposition 3.19, and so is $\iota_{w, !} 1 \star \iota_{w^{\prime}, !} 1$ as a direct summand. This proves (3.18).

We now prove Theorem 3.17 in the general case. By definition, the category DTM

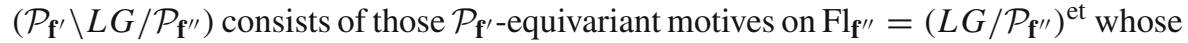
underlying non-equivariant motive is a Tate motive with respect to the stratification

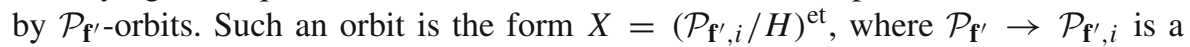
smooth $S$-affine quotient (i.e., of finite type) and $H \subset \mathcal{P}_{\mathbf{f}^{\prime}, i}$ is a smooth closed subgroup scheme with connected fibers over $S$. Let $e: S \rightarrow X$ be the unit section. The composition

$$
\mathrm{DM}_{\mathcal{P}_{\mathbf{f}^{\prime}}}(X) \simeq \mathrm{DM}_{\mathcal{P}_{\mathbf{f}^{\prime}, i}}(X) \stackrel{e^{!}}{\rightarrow} \mathrm{DM}_{H}(S)
$$

which is an equivalence by [60, Lem. 2.2.21], restricts to an equivalence $e^{!}: \operatorname{DTM}_{\mathcal{P}_{\mathbf{f}^{\prime}}}(X) \simeq \operatorname{DTM}_{H}(S)$ on Tate motives by [60, Prop. 3.1.23]. Thus, for a

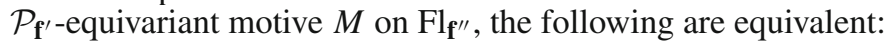


- $M$ is Tate with respect to the stratification by $\mathcal{P}_{\mathbf{f}^{\prime} \text {-orbits; }}$

- $M$ is Tate at the base point of each $\mathcal{P}_{\mathbf{f}^{\prime}}$-orbit;

- $M$ is Tate with respect to the (finer) stratification by $\mathcal{B}$-orbits.

We may therefore assume that $\mathcal{P}_{\mathbf{f}^{\prime}}=\mathcal{B}$ is the standard Iwahori. Using that $\operatorname{DTM}\left(\mathcal{B} \backslash L G / \mathcal{P}_{\mathbf{f}^{\prime \prime}}\right)$ consists of $\mathcal{P}_{\mathbf{f}^{\prime \prime}}$-equivariant motives whose underlying motive on $\mathrm{Fl}^{\mathrm{op}}=(\mathcal{B} \backslash L G)^{\mathrm{et}}$ is stratified Tate $[60,5.3 .4]$, we similarly reduce to the case that $\mathcal{P}_{\mathbf{f}^{\prime \prime}}=\mathcal{B}$ and therefore deduce Theorem 3.17 in general from Proposition 3.26.

\section{Purity of Tate motives}

Throughout Sect. 4, the base scheme $S$ is as in Notation 2.1 .

\subsection{Intersection motives are pure}

In this section, we show that the intersection motives $\mathrm{IC}_{w}$ for the stratification of $\mathrm{Fl}_{\mathbf{f}}$ given by the $\mathcal{P}_{\mathbf{f}^{\prime}}$-orbits (for arbitrary facets $\mathbf{f}, \mathbf{f}^{\prime}$ contained in the closure of the base alcove $\mathbf{a}_{0}$ ) are pure. This will be proven by lifting the corresponding fact for $\ell$-adic intersection complexes to motives over $\mathbf{F}_{p}$, which is then extended to more general base schemes using the results of Sect. 2.4.

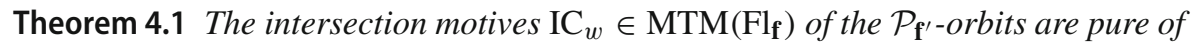
weight $\operatorname{dim} \mathrm{Fl}_{\mathbf{f}}^{w}$. (Here purity refers to the weight structure for motives on ind-schemes, established in [60, Theorem 2.4.2].)

Proof Let $S=\operatorname{Spec} \mathbf{F}_{p}$ first. Pick some prime $\ell$ invertible in $\mathbf{F}_{p}$. The $\ell$-adic realization $\rho_{\ell}: \mathrm{DM}\left(\mathrm{Fl}_{\mathbf{f}}\right)^{\mathrm{c}} \rightarrow \mathrm{D}_{\mathrm{ct}}^{\mathrm{b}}\left(\mathrm{Fl}_{\mathbf{f}}, \mathbf{Q}_{\ell}\right)$ takes values in the subcategory $\mathrm{D}_{\mathrm{et} \text {, mix }}\left(\mathrm{Fl}_{\mathbf{f}}, \mathbf{Q}_{\ell}\right)$ of mixed complexes. With respect to the standard weight structure on that category (and the motivic weight structure on DM), the functor is weight-exact. (This follows from the definition of the motivic weight structure and the fact that in the realization these functors preserve weights, see [10, Prop. 3.6.1.2] for details.) Its restriction to $\operatorname{DTM}\left(\mathrm{Fl}_{\mathbf{f}}\right)$ is t-exact and conservative by [60, Lem. 3.2.8]. It therefore creates the $t$-structure and the weight structure (Lemma 2.17). Now recall the notation (2.2). Since $\rho_{\ell}$ also commutes with $\left(l_{w}\right)_{\text {! }}$ and $\left(\iota_{w}\right)_{*}$, the motive $\mathrm{IC}_{w}$ is mapped under $\rho_{\ell}$ to the $\ell$-adic intersection complex on the Schubert variety $\mathrm{Fl}_{\mathbf{f}}^{\leq w}$, i.e., to $\rho_{\ell}\left(\mathrm{IC}_{w}\right)=\left(i_{w}\right)_{*}\left(j_{w}\right)_{!_{*}}\left(\mathbf{Q}_{\ell}\left[\operatorname{dim}\left(\mathrm{Fl}_{\mathbf{f}}^{w}\right)\right]\right)$. By [44, Ex. III.10.3], it is pure of weight $+\operatorname{dim} \mathrm{Fl}_{\mathbf{f}}^{w}$, hence so is $\mathrm{IC}_{w}$ itself.

For general $S$, consider the zig-zag $S \rightarrow \operatorname{Spec} \mathbf{Z} \leftarrow \operatorname{Spec} \mathbf{F}_{p}$. By Theorem 2.14 and (2.16), the purity of $\operatorname{IC}_{w, S} \in \operatorname{MTM}\left(\mathrm{Fl}_{\mathbf{f}, S}\right)$ is equivalent to the one of $\operatorname{IC}_{w, \mathbf{Z}} \in$

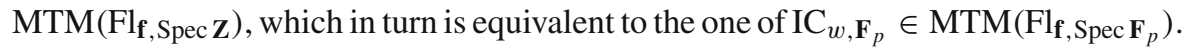

Corollary 4.2 There is a functorial weight filtration for any object $M \in \mathrm{MTM}\left(\mathrm{Fl}_{\mathbf{f}}\right)$

$$
0=M_{-\infty} \subset \cdots \subset M_{0} \subset M_{1} \subset \ldots M_{\infty}=M
$$


such that $\operatorname{gr}_{i}^{\mathrm{W}}(M):=M_{i} / M_{i-1} \in \mathrm{MTM}\left(\mathrm{Fl}_{\mathbf{f}}\right)$ is pure of weight $i($ for $-\infty<i<\infty)$. If $M$ is compact, this filtration is finite, and the $\mathrm{gr}_{i}^{\mathrm{W}}(M)$ are compact.

Proof Let $\mathrm{MTM}_{i}:=\left\{\mathrm{IC}_{w}(n), \operatorname{dim} \mathrm{Fl}_{\mathbf{f}}^{w}=i+2 n\right\}$. The collection of these subcategories forms a semi-orthogonal family in the sense of [9, Def. 1.1.4]: $\operatorname{Hom}_{\mathrm{DTM}(\mathrm{Gr})}\left(\mathrm{MTM}_{i}, \mathrm{MTM}_{j}[s]\right)$ vanishes for $s<0$ by the existence of the perverse motivic t-structure. It vanishes for $s>i-j$ for weight reasons, noting that $\operatorname{MTM}_{j}[s]$ consists of objects which are pure of weight $j+s$ by Theorem 4.1. The claim for compact objects then follows from [9, Thm. 1.2.1, (ii'), (iv')]. In general, any object $M \in \mathrm{MTM}$ is the filtered colimit of its compact subobjects $M^{(n)} \subset M$, so that we obtain a (possibly infinite) weight filtration of $M$ by taking the colimit of the weight filtrations of the $M^{(n)}$.

\subsection{Convolution preserves weights}

In this section, we show that the convolution product preseves the subcategories of motives of weight $\leq n$ and $\geq n$. To prove this, we need the following lemma:

Lemma 4.3 For separated schemes $X_{1}, X_{2}$ of finite type over a perfect field $k$, the exterior product

$$
\otimes: \operatorname{DM}\left(X_{1}\right) \times \operatorname{DM}\left(X_{2}\right) \rightarrow \operatorname{DM}\left(X_{1} \times_{k} X_{2}\right)
$$

is weight-exact.

Proof By the description of the $\leq 0$ - and $\geq 0$-part of the weight structure in [36, Rem. 1.17], we have to show that exterior products of motives of the form $\left(f_{i}\right) ! 1(n)[2 n]$, where $f_{i}: T_{i} \rightarrow X_{i}$ is a proper map and $T_{i}$ is regular for $i=1,2$, is again pure of weight 0 .

Since $k$ is perfect, a finite type $k$-scheme is regular iff it is smooth over $k$ [64, Tag $0 \mathrm{~B} 8 \mathrm{X}]$. Hence $T_{1} \times_{k} T_{2}$ is again regular. We conclude using the formula $f_{1, !} 1 \otimes f_{2, !} 1=$ $\left(f_{1} \times f_{2}\right)$ ! 1 whose proof is straightforward using the base change formula for $f^{*} \mathrm{vs}$. $g$ ! and the projection formula (see [60, Synopsis 2.1.1, (vii) and (x)]).

Remark 4.4 If $k$ is an imperfect field, Lemma 4.3 still holds by virtue of the equivalence

$$
\mathrm{DM}\left(X_{i}\right) \cong \mathrm{DM}\left(X_{i} \times_{k} \hat{k}\right)
$$

for some perfect closure $\hat{k}$ of $k$. The stronger corresponding statement for $\mathrm{SH}\left[\frac{1}{\operatorname{char} k}\right]$ instead of DM is shown in [22, Cor. 2.1.7]. The equivalence is also shown in [59, $\S 2.2]$. This equivalence is compatible with $\otimes$ and !-pushforwards, so we obtain our claim in this case.

In order to state that the convolution product preserves weights, we need to talk about weights on equivariant motives. The idea is simple: a $G$-equivariant motive is declared to be of weights $\geq 0$ or $\leq 0$ if its corresponding underlying non-equivariant motive has the corresponding property. The following definition makes this precise. 
Note that we only define a pair of full subcategories of $\mathrm{DM}(G \backslash X)$. We do not claim they constitute a weight structure, i.e., we do not assert the existence of weight truncation triangles.

Definition 4.5 Suppose a smooth group scheme $G$ acts on a scheme $X$ (both supposed to be separated and of finite type over $S$ ). We define

$$
\begin{aligned}
& \operatorname{DM}(G \backslash X)^{\mathrm{w} \leq 0}:=\lim \mathrm{DM}^{!, \mathrm{w} \leq 0}(\operatorname{Bar}(G, X)) \\
& \operatorname{DM}(G \backslash X)^{\mathrm{w} \geq 0}:=\lim \mathrm{DM}^{!, \mathrm{w} \geq 0}(\operatorname{Bar}(G, X)),
\end{aligned}
$$

i.e., we apply $\mathrm{DM}(-)^{\mathrm{w} \leq 0}$ to each term in the bar construction (see around $[60$, Def. 2.2.6]), with transition functors given by !-pullback.

Remark 4.6 The smooth transition maps preserve the subcategory of objects of weight $\geq 0$ and also $\leq 0$, so the limits make sense.

The definition is independent of the choice of the presentation of $G \backslash X$ : if $G \backslash X=$ $G^{\prime} \backslash X^{\prime}$, then $M \in \operatorname{DM}(G \backslash X)$ is of weights $\leq 0$, say, if it is so on $X$ (under !-pullback) and therefore on $G^{\prime} \times X=G \times X^{\prime}$. Here and in the following we use the standard weight preservation properties under smooth pullback and (in (4.7) below) also under proper pushforward [36, Thm. 3.8]. The projection map $G \times X^{\prime} \rightarrow X^{\prime}$ is smooth and surjective, hence $\left.M\right|_{X^{\prime}}$ is of weights $\leq 0$ by Lemma 2.17 .

The following weight preservation property will be central to the stability of the Satake category Sat ${ }_{G}$ under convolution (see Lemma 6.5). We only consider compact objects since it is enough for our purposes, but the statement could be extended to arbitrary ones, at the expense of a more lengthy discussion of weights in that case. Recall from [60, Cor. 2.3.4 and Prop. 2.2.11] that

$$
\operatorname{DM}\left(\mathcal{P}_{\mathbf{f}} \backslash \mathrm{Fl}_{\mathbf{f}}\right)^{\mathrm{c}}=\operatorname{colim}_{\left(t_{i j}\right) !} \operatorname{DM}\left(\mathcal{P}_{\mathbf{f}} \backslash \mathrm{Fl}_{\mathbf{f}, i}\right)^{\mathrm{c}}=\operatorname{colim}_{\left(t_{i j}\right) !} \operatorname{DM}\left(\mathcal{P}_{\mathbf{f}, i} \backslash \mathrm{Fl}_{\mathbf{f}, i}\right)^{\mathrm{c}}
$$

where $\mathrm{Fl}_{\mathbf{f}}=$ colim $\mathrm{Fl}_{\mathbf{f}, i}$ is a presentation as an ind-scheme (with transition maps denoted by $t_{i j}$ ) and $\mathcal{P}_{\mathbf{f}, i}$ is an appropriate finite-type quotient of $\mathcal{P}_{\mathbf{f}}$ acting on $\mathrm{Fl}_{\mathbf{f}, i}$, and colim denotes the colimit in the $\infty$-category of $\infty$-categories, which (see loc. cit.) can in this case just be thought of as the union of the above $\infty$-categories, as $i$ grows. Using that $\left(t_{i j}\right)$ ! is weight-exact, we define

$$
\operatorname{DM}\left(\mathcal{P}_{\mathbf{f}} \backslash \mathrm{Fl}_{\mathbf{f}}\right)^{\mathrm{c}, \mathrm{w} \leq 0}:=\operatorname{colim}_{\left(t_{i j}\right) !} \operatorname{DM}\left(\mathcal{P}_{\mathbf{f}, i} \backslash \mathrm{Fl}_{\mathbf{f}, i}\right)^{\mathrm{c}, \mathrm{w} \leq 0}
$$

and likewise for $\geq 0$.

Proposition 4.8 The convolution product for Tate motives is weight-exact, i.e., for any objects $A, B \in \operatorname{DTM}\left(\mathcal{P}_{\mathbf{f}} \backslash \mathrm{Fl}_{\mathbf{f}}\right)^{\mathrm{c}, \mathrm{w} \leq 0}$, their convolution $A \star B$ is also of weights $\leq 0$ and likewise with $\geq 0$.

Proof We first assume $S$ is (the spectrum of) a perfect field $k$ or just $S=\operatorname{Spec} \mathbf{F}_{p}$, in which case we show the stronger statement that the convolution product on $\operatorname{DM}\left(\mathcal{P}_{\mathbf{f}} \backslash \mathrm{Fl}_{\mathbf{f}}\right)^{\mathrm{c}}$ (as opposed to DTM) preserves weights. We use the notation in the 
diagram (3.10) and the discussion around it. The functor $-\otimes-: \operatorname{DM}(X) \times \operatorname{DM}\left(\mathcal{P}_{\mathbf{f}, i} \backslash\right.$ $Y) \rightarrow \operatorname{DM}\left(X \times \mathcal{P}_{\mathbf{f}, i} \backslash Y\right)$ preserves weights by Definition 4.5 and Lemma 4.3. Similarly, $(e \circ \tilde{p})^{!}$preserves weights: $e \circ \tilde{p}$ is a smooth map of prestacks, i.e., it admits a smooth covering on which the map is a smooth map of finite type $k$-schemes. Hence $(e \circ \tilde{p})^{\text {! }}$ preserves weights by the same argument as in Definition 4.5. Finally, $\tilde{m}$ is proper so that $\tilde{m}$ ! preserves weights again. Hence the non-equivariant motive underlying $A \star B$, namely $\tilde{m}_{!}(A \widetilde{\otimes} B)$ has the same weights as $A \otimes B$, so that the same holds for $A \star B$ itself.

For general $S$, we consider parallely the structural map $f: T:=S \rightarrow \operatorname{Spec} \mathbf{Z}$ and the closed immersion $f: T:=\operatorname{Spec} \mathbf{F}_{p} \rightarrow \operatorname{Spec} \mathbf{Z}$. The functor $f^{*}:$ $\operatorname{DTM}\left(\mathcal{P}_{\mathbf{f}, \mathbf{Z}} \backslash L G_{\mathbf{Z}} / \mathcal{P}_{\mathbf{f}, \mathbf{Z}}\right) \rightarrow \operatorname{DTM}\left(\mathcal{P}_{\mathbf{f}, T} \backslash L G_{T} / \mathcal{P}_{\mathbf{f}, T}\right)$ creates the weight structure by Theorem 2.14. We conclude by using Lemma 3.15 .

\section{Mixed Tate motives on the affine Grassmannian}

In this section, we endow the category $\operatorname{MTM}\left(L^{+} G \backslash L G / L^{+} G\right)$ with a Tannakian structure, cf. Theorem 5.14. A recurrent idea is that the conservativity of the $\ell$-adic realization functor restricted to stratified Tate motives allows us to lift many statements from the $\ell$-adic to the motivic setting.

Synopsis 5.1 Throughout Sect. 5, the base scheme $S$ is as in Notation 2.1. We fix a split reductive group $G \rightarrow S$ and a Borel pair $T \subset B \subset G$ over $S$ where $T$ is a split maximal torus contained in the Borel subgroup $B$. We start by listing some basic properties as needed in the following, see Sect. 2 for more details and the references cited there.

(i) The affine Grassmannian $\mathrm{Gr}=\mathrm{Gr}_{G} \rightarrow S$ is the quotient of ordinary étale sheaves $\left(L G / L^{+} G\right)^{\text {et }}$ with base point denoted by $e \in \operatorname{Gr}(S)$. For each dominant cocharacter $\mu \in X_{*}(T)^{+}$, the locally closed immersion of the $L^{+} G$-orbit of $\varpi^{\mu} \cdot e$ is denoted by

$$
\iota_{\mu}: \mathrm{Gr}^{\mu} \stackrel{j_{\mu}}{\hookrightarrow} \mathrm{Gr} \leq \mu \stackrel{i_{\mu}}{\hookrightarrow} \mathrm{Gr}
$$

Each Schubert scheme $\mathrm{Gr} \leq \mu \rightarrow S$ is proper, and the open orbit $\mathrm{Gr}^{\mu} \subset \mathrm{Gr} \leq \mu$ is $S$ smooth, fibrewise dense with geometrically connected fibers of relative dimension $\langle 2 \rho, \mu\rangle \in \mathbf{Z}_{\geq 0}$. For $\lambda, \mu \in X_{*}(T)^{+}$, we have $\mathrm{Gr}^{\lambda} \subset \mathrm{Gr}^{\leq} \leq \mu$ if and only if $\lambda \leq \mu$ in the dominance partial order on $X_{*}(T)^{+}$. For details see [60, Exam. 4.2.16 and Lem. 4.3.7].

(ii) Throughout, we work with the prestack double quotient $L^{+} G \backslash L G / L^{+} G \rightarrow S$, cf. [60, 5.3]. For each point $s \in S$, there is a homeomorphism of topological spaces

$$
\left|L^{+} G_{S} \backslash L G_{S} / L^{+} G_{S}\right| \simeq X_{*}\left(T_{S}\right)^{+} \stackrel{S \text { connected }}{=} X_{*}(T)^{+},
$$

where $X_{*}(T)^{+}$is endowed with the topology given by the dominance partial order " $\leq$ ", i.e., for each $\mu \in X_{*}(T)^{+}$the subset $\{\lambda \mid \lambda \leq \mu\}$ is closed. 
(iii) The étale descent equivalence $\mathrm{DM}\left(L^{+} G \backslash L G / L^{+} G\right)=\mathrm{DM}\left(L^{+} G \backslash \mathrm{Gr}\right)$ (cf. Theorem 2.6) will be used freely throughout. We have the full subcategory

$$
\operatorname{DTM}\left(L^{+} G \backslash L G / L^{+} G\right) \subset \operatorname{DM}\left(L^{+} G \backslash L G / L^{+} G\right)
$$

of stratified Tate motives. For each $\mu \in X_{*}(T)^{+}$, there is an equivalence $\operatorname{DTM}\left(L^{+} G \backslash \mathrm{Gr}^{\mu}\right)=\operatorname{DTM}\left(H_{\mu} \backslash S\right)$ where $H_{\mu}=L^{+} G \cap\left(\varpi^{\mu} L^{+} G \varpi^{-\mu}\right)$ denotes the stabilizer, cf. [60, Prop. 3.1.23]. Here $H_{\mu} \rightarrow S$ is a fibrewise connected, strictly pro-algebraic closed subgroup of $L^{+} G$ by [60, Lem. 4.3 .7 (ii)], that is, it can be written as a sequential limit of fiberwise connected, $S$-smooth, $S$-affine group schemes with surjective transition maps.

(iv) The category of stratified Tate motives admits a non-degenerate $t$-structure whose heart is the full subcategory of mixed Tate motives

$$
\operatorname{MTM}\left(L^{+} G \backslash L G / L^{+} G\right) \subset \operatorname{DTM}\left(L^{+} G \backslash L G / L^{+} G\right) .
$$

This category is abelian, Q-linear, and the forgetful functor

$$
\operatorname{MTM}\left(L^{+} G \backslash L G / L^{+} G\right) \longrightarrow \operatorname{MTM}(\mathrm{Gr})
$$

is fully faithful and induces a bijection on simple objects by Theorem 2.8. (In fact, it is an equivalence by Corollary5.7 below.) The simple objects are the intersection motives $\operatorname{IC}_{\mu}(n)$ for $\mu \in X_{*}(T)^{+}, n \in \mathbf{Z}$. These are pure of weight $\langle 2 \rho, \mu\rangle-2 n \in \mathbf{Z}$ by Theorem 4.1. The closed set $\{\lambda \mid \lambda \leq \mu\} \subset X_{*}(T)^{+}$ identifies with the closure of the support of $\operatorname{IC}_{\mu}(n)$ for any $n \in \mathbf{Z}$, cf. [60, Lem. 4.2.11, 4.3.7]. Furthermore, for each $\mu \in X_{*}(T)^{+}$, there are equivalences $\operatorname{MTM}\left(\mathrm{Gr}^{\mu}\right)=\operatorname{MTM}\left(L^{+} G \backslash \mathrm{Gr}^{\mu}\right)=\operatorname{MTM}\left(H_{\mu} \backslash S\right)=\operatorname{MTM}(S)$ by Lemma 2.9 and [60, Cor. 3.2.21].

(v) The convolution product defines a functor

$$
\text { - - : } \operatorname{DTM}\left(L^{+} G \backslash L G / L^{+} G\right) \times \operatorname{DTM}\left(L^{+} G \backslash L G / L^{+} G\right) \longrightarrow \operatorname{DTM}\left(L^{+} G \backslash L G / L^{+} G\right)
$$

on the category of stratified Tate motives, cf. Theorem 3.17. (We show in Lemma 5.8 below that the convolution product preserves the subcategory $\operatorname{MTM}\left(L^{+} G \backslash L G / L^{+} G\right)$. Moreover, the two possible approaches in defining the convolution product discussed in Proposition 3.4 are isomorphic on this category.)

(vi) For each geometric point $f: \bar{s} \rightarrow S$, the fibre of the $\ell$-adic realization functor (cf. Corollary 2.20)

$$
\rho_{\ell, \bar{s}}:=f^{*} \circ \rho_{\ell}: \operatorname{MTM}(\mathrm{Gr})^{\mathrm{c}} \rightarrow \operatorname{Perv}\left(\mathrm{Gr}_{\bar{s}}, \mathbf{Q}_{\ell}\right)
$$

is exact, conservative and faithful. Under the realization, the motivic convolution product corresponds to the classical convolution product in the $\ell$-adic setting (Proposition 3.14). 


\subsection{Indecomposable objects}

The affine Grassmannian admits a decomposition into open and closed sub-indschemes $\mathrm{Gr}=\sqcup_{\tau \in \pi_{1}(G)} \mathrm{Gr}_{\tau}$ where $\pi_{1}(G)$ is the algebraic fundamental group, i.e., the finitely generated abelian group given by the quotient of the cocharacter lattice by the coroot lattice. Within each component $\mathrm{Gr}_{\tau}$, every Schubert cell $\mathrm{Gr}^{\mu} \rightarrow S$ has either even- or odd-dimensional fibers: indeed if $\lambda, \mu \in X_{*}(T)^{+}$with the same class in $\pi_{1}(G)$, then $\langle 2 \rho, \lambda\rangle \equiv\langle 2 \rho, \mu\rangle$ in $\mathbf{Z} / 2$ because $\rho$ takes integer values on the coroot lattice. This defines the locally constant function

$$
X_{*}(T)^{+} \rightarrow \mathbf{Z} / 2, \quad \mu \mapsto\langle 2 \rho, \mu\rangle \bmod 2,
$$

where the source is equipped with the topology given by the dominance order as in Synopsis 5.1 (ii). An object $A \in \mathrm{MTM}(\mathrm{Gr})$ is said to have constant parity $p(A) \in \mathbf{Z} / 2$ if the restriction of (5.2) to the closure of its support is a constant function, i.e., the object is supported either on a union of even components, or is supported on a union of odd components. The following result is a direct consequence of our discussion and the Kazhdan-Lusztig parity vanishing.

\section{Corollary 5.3 Let $A \in \mathrm{MTM}(\mathrm{Gr})$.}

(i) There exists a canonical decomposition $A=A_{\mathrm{even}} \oplus A_{\mathrm{odd}}$ into objects of even and odd constant parity.

(ii) If $A \in \operatorname{MTM}(\mathrm{Gr})$ is of constant parity $p(A) \in \mathbf{Z} / 2$, then

$$
{ }^{\mathrm{cl}} \mathrm{H}^{i}(A)=0, \quad \text { whenever } i \not \equiv p(A) \quad \bmod 2,
$$

where ${ }^{\mathrm{cl}} \mathrm{H}^{i}$ denotes the truncation with respect to the classical motivic $t$-structure on $\mathrm{Gr}$.

(iii) If $\iota: X \subset \mathrm{Gr}$ is a finite union of Schubert schemes, then

$$
{ }^{\mathrm{m}} \mathrm{H}^{i}\left(\iota^{*} A\right)=0, \quad \text { whenever } i \not \equiv 0 \quad \bmod 2,
$$

where ${ }^{\mathrm{m}} \mathrm{H}^{i}$ denotes the truncation with respect to the perverse motivic $t$-structure on $X$.

Proof Part (i) is immediate from the definitions using that each connected component of $\mathrm{Gr}$ is either of constant even or constant odd parity. Part (ii) and (iii) for $\ell$-adic sheaves are certainly well-known, and hence are immediate from the conservativity of $\rho_{\ell, \bar{s}}$ (Synopsis 5.1 (vi)). Let us give an argument by reduction to Theorem 2.21. The category $\operatorname{MTM}(\mathrm{Gr})$ is compactly generated, so $A$ is the filtered colimit of its compactly generated subobjects. Moreover, ${ }^{\mathrm{cl}} \mathrm{H}$ and ${ }^{\mathrm{m}} \mathrm{H}$ commute with filtered colimits, so we may assume $A$ is compact. For (ii), we use that the length function on $X_{*}(T)^{+}=$ $W_{0} \backslash W / W_{0}$ is computed as $l(\mu)=\langle 2 \rho, \mu\rangle$ which is also the relative dimension of each $\mathrm{Gr}^{\mu} \rightarrow S$. Hence, for each $\mu \in X_{*}(T)^{+}$, the vanishing of ${ }^{{ }^{c l}} \mathrm{H}^{i}\left(\iota_{\mu}^{*} A\right) \in$ $\operatorname{DTM}_{L^{+} G}\left(\mathrm{Gr}^{\mu}\right)=\operatorname{DTM}_{H_{\mu}}(S)$ (Synopsis 5.1 (iii)) in degrees $i$ as above follows 
from Theorem 2.21. Here we use that the forgetful functor $\operatorname{DM}_{H_{\mu}}(S) \rightarrow \mathrm{DM}(S)$ is conservative. By the compactness of $A$, its support is a finite-type subscheme of $\mathrm{Gr}$. We can therefore invoke the localization property of DM to see that the condition

$$
{ }^{\mathrm{cl}} \mathrm{H}^{i}\left(\iota_{\mu}^{*} A\right)=\iota_{\mu}^{* \mathrm{cl}} \mathrm{H}^{i}(A)=0
$$

for all $\mu \in X_{*}(T)^{+}$implies the vanishing ${ }^{\mathrm{c}} \mathrm{H}^{i}(A)=0$. For (iii), the argument is similar now taking the dimension shifts in the construction of the perverse motivic $t$-structure into account.

The next lemma addresses the interplay of Tate motives on the base scheme $S$ and intersection motives. For $L \in \operatorname{MTM}(S)$ and $\mu \in X_{*}(T)^{+}$, we write

$$
\mathrm{IC}_{L, \mu} \stackrel{\text { def }}{=}\left(i_{\mu}\right)_{*}\left(j_{\mu}\right)_{! *} L\left[\operatorname{dim} \mathrm{Gr}^{\mu}\right] \in \operatorname{MTM}(\mathrm{Gr})
$$

for the intersection motive twisted by the motive $L$ (more precisely by its $*$-pullback along the projection $\mathrm{Gr}^{\mu} \rightarrow S$ ). We have $\mathrm{IC}_{1_{S}(n), \mu}=\mathrm{IC}_{\mu}(n)$ for any $n \in \mathbf{Z}$.

Lemma 5.4 For any $L \in \operatorname{MTM}(S), \mu \in X_{*}(T)^{+}$, there is a canonical isomorphism

$$
\mathrm{IC}_{L, 0} \star \mathrm{IC}_{1_{S}, \mu} \simeq \mathrm{IC}_{L, \mu}
$$

Proof Let $i: S=\mathrm{Gr}=0 \rightarrow \mathrm{Gr}$ denote the inclusion of the base point, so that $\mathrm{IC}_{L, 0}=$ $i_{*} L$. In the notation of (3.10), both the map $\tilde{m}$ and the map $e \circ \tilde{p}$ are isomorphisms when restricted to $\mathrm{Gr} \leq 0 \widetilde{\times} \mathrm{Gr}^{\leq \mu}=\mathrm{Gr} \leq \mu$. Thus $\mathrm{IC}_{L, 0} \star \mathrm{IC}_{1_{S}, \mu}=i_{*} L \widetilde{\otimes} \mathrm{IC}_{\mu}=p_{\mu}^{*} L \otimes \mathrm{IC}_{\mu}=$ $\mathrm{IC}_{L, \mu}$ where $p_{\mu}: \mathrm{Gr}^{\leq \mu} \rightarrow S$. The last equality is checked using the characterization of IC-motives in [60, Lem. 3.3.3].

Corollary 5.5 Let $L, L^{\prime} \in \operatorname{MTM}(S)$ and $\lambda, \mu \in X_{*}(T)^{+}$. Then

$$
\operatorname{Ext}_{\mathrm{MTM}(\mathrm{Gr})}^{1}\left(\mathrm{IC}_{L, \lambda}, \mathrm{IC}_{L^{\prime}, \mu}\right)= \begin{cases}\operatorname{Ext}_{\mathrm{MTM}(S)}^{1}\left(L, L^{\prime}\right), & \text { if } \lambda=\mu \\ 0, & \text { else }\end{cases}
$$

In particular, if $\operatorname{MTM}(S)$ is semisimple (e.g. $S=\operatorname{Spec}\left(\mathbf{F}_{q}\right)$ ), then $\operatorname{MTM}(\mathrm{Gr})$ is semisimple as well.

Proof This is immediate from Corollary 5.3 (iii), and we refer to [26, Prop. $1 \mathrm{ff}$.] and [57, Prop. 3.1] for more details. Here is a sketch for the reader's convenience: First assume $\mu=\lambda$, and denote $\mathrm{IC}:=\mathrm{IC}_{L, \mu}, \mathrm{IC}^{\prime}:=\mathrm{IC}_{L^{\prime}, \lambda}$. Let $\mathrm{Gr}^{\mu} \stackrel{j}{\rightarrow} \mathrm{Gr}^{\leq} \mu \stackrel{i}{\leftarrow}$ $\mathrm{Gr}^{\leq} \leq \mu \backslash \mathrm{Gr}^{\mu}$. We have a long exact localization sequence

$$
\begin{aligned}
\cdots & \rightarrow \operatorname{Hom}\left(\mathrm{IC}, i_{!} i ! \mathrm{IC}^{\prime}[1]\right) \rightarrow \operatorname{Hom}\left(\mathrm{IC}, \mathrm{IC}^{\prime}[1]\right) \\
& \rightarrow \operatorname{Hom}\left(\mathrm{IC}, j_{*} j^{*} \mathrm{IC}^{\prime}[1]\right) \rightarrow \operatorname{Hom}\left(\mathrm{IC}, i_{!} i^{!} \mathrm{IC}^{\prime}[2]\right) \rightarrow \ldots
\end{aligned}
$$

We have the following isomorphisms:

$$
\operatorname{Hom}\left(\mathrm{IC}^{\prime}, j_{*} j^{*} \mathrm{IC}^{\prime}[1]\right)=\operatorname{Hom}\left(j^{*} \mathrm{IC}, j^{*} \mathrm{IC}^{\prime}[1]\right)
$$




$$
=\operatorname{Ext}_{\mathrm{MTM}\left(\mathrm{Gr}^{\mu}\right)}^{1}\left(L, L^{\prime}\right)=\operatorname{Ext}_{\operatorname{MTM}(S)}^{1}\left(L, L^{\prime}\right)
$$

where the last one is a consequence of the equivalence $\operatorname{MTM}(S) \simeq \operatorname{MTM}\left(\mathrm{Gr}^{\mu}\right)$ (Synopsis 5.1 (iv)). It is therefore enough to show that the outer groups in the above exact sequence vanish. The Kazhdan-Lusztig parity vanishing implies that $i^{*} \mathrm{IC}$, resp. $i^{*} \mathrm{IC}^{\prime}$ lives in perverse degree $\leq-2$. Indeed, by general properties it lives in degree $\leq 0$, degree 0 vanishes because it is an IC-sheaf, degree -1 vanishes by Corollary 5.3 (iii). By duality $i ! \mathrm{IC}^{\prime}$ lives in perverse degrees $\geq 2$, and taking the shifts [1], resp. [2] into account, we see that the outer groups vanish by the axioms of a $t$-structure. This implies the corollary in the case $\mu=\lambda$. Now let $\mu<\lambda$, and denote $i: \operatorname{Gr} \leq \mu \rightarrow \mathrm{Gr} \leq \lambda$ the closed embedding. Again the group

$$
\operatorname{Hom}\left(i_{*} \mathrm{IC}_{L, \mu}, \mathrm{IC}_{L^{\prime}, \lambda}[1]\right)=\operatorname{Hom}\left(\mathrm{IC}_{L, \mu}, i ! \mathrm{IC}_{L^{\prime}, \lambda}[1]\right)
$$

vanishes for $t$-structure reasons as above using the parity vanishing. The case $\lambda<\mu$ is similar. Now if both $\mu \not \leq \lambda$ and $\lambda \not \leq \mu$, there are no extensions between IC-sheaves. This is shown in loc. cit. without appealing to parity vanishing.

The category of compact objects $\operatorname{MTM}(\mathrm{Gr})^{\mathrm{c}}$ is both Noetherian and Artinian (Theorem 2.8), i.e., each object has finite length. Thus, it is a Krull-Remak-Schmidt category by [45], so that each object $A \in \operatorname{MTM}(\mathrm{Gr})^{\mathrm{c}}$ admits a direct sum decomposition into indecomposable objects $A=A_{1} \oplus \ldots \oplus A_{n}$ which is unique up to permutation of the factors.

Corollary 5.6 Let $A \in \mathrm{MTM}(\mathrm{Gr})^{\mathrm{c}}$. Then there exist $L_{1}, \ldots, L_{n} \in \operatorname{MTM}(S)^{\mathrm{c}}$ indecomposable, and $\mu_{1}, \ldots, \mu_{n} \in X_{*}(T)^{+}$such that $A \simeq \oplus_{i=1, \ldots, n} \mathrm{IC}_{L_{i}, \mu_{i}}$. Further, each $\mathrm{IC}_{L_{i}, \mu_{i}}$ is simple if and only if $L_{i} \simeq 1_{S}\left(n_{i}\right)$ for some $n_{i} \in \mathbf{Z}$.

Proof By the discussion above, we may assume that $A$ is indecomposable in which case we have to show $A \simeq \operatorname{IC}_{L, \mu}$ for some necessarily indecomposable $L \in \operatorname{MTM}^{\mathrm{c}}(S)$ and $\mu \in X_{*}(T)^{+}$. We proceed by induction on the length $l(A)$. The condition $l(A)=1$ is equivalent to $A$ being simple, and thus $A \simeq \mathrm{IC}_{L, \mu}$ with $L=1(n)$ for some $n \in \mathbf{Z}$ (Synopsis 5.1 (iv)). Let $l(A) \geq 2$, and let $0 \neq A^{\prime} \subset A$ be a subobject of length $l\left(A^{\prime}\right)=$ 1. In particular, $A^{\prime}$ is indecomposable. The quotient $A / A^{\prime}$ is also indecomposable. By induction $A^{\prime} \simeq \mathrm{IC}_{L^{\prime}, \mu}, A / A^{\prime} \simeq \mathrm{IC}_{L, \lambda}$, and thus $[A] \in \operatorname{Ext}_{\mathrm{MTM}(\mathrm{Gr})}^{1}\left(\mathrm{IC}_{L, \lambda}, \mathrm{IC}_{L^{\prime}, \mu}\right)$. As $A$ is indecomposable, the class $[A] \neq 0$ which by Corollary 5.5 implies that $\lambda=\mu$ and that $A$ is of the desired form.

The following result is similar to [52, Prop. 2.1].

Corollary 5.7 The forgetful functor

$$
\operatorname{MTM}\left(L^{+} G \backslash L G / L^{+} G\right) \stackrel{\simeq}{\longrightarrow} \operatorname{MTM}(\mathrm{Gr})
$$

is an equivalence of $\mathbf{Q}$-linear abelian categories.

Proof The functor is fully faithful by [60, 5.3.4 (iii)]. As every object in MTM(Gr) is isomorphic to a direct sum of objects $\mathrm{IC}_{L, \mu}$, it is essentially surjective as well. 


\subsection{Tensor structure}

In this section, we show that the convolution product on $\operatorname{DM}\left(L^{+} G \backslash L G / L^{+} G\right)$ preserves the subcategory $\operatorname{MTM}\left(L^{+} G \backslash L G / L^{+} G\right)$.

Lemma 5.8 For $A, B \in \operatorname{MTM}\left(L^{+} G \backslash L G / L^{+} G\right)$ one has $A \star B \in \operatorname{MTM}\left(L^{+} G \backslash L G /\right.$ $\left.L^{+} G\right)$, i.e., the category $\operatorname{MTM}\left(L^{+} G \backslash L G / L^{+} G\right)$ is stable under the convolution product.

Proof By Theorem 3.17, convolution preserves Tateness, i.e., $A \star B \in \operatorname{DTM}\left(L^{+} G \backslash\right.$ $\left.L G / L^{+} G\right)$. The remaining property $A \star B \in \operatorname{MTM}\left(L^{+} G \backslash L G / L^{+} G\right)$, equivalently ${ }^{m} \mathrm{H}^{i}(A \star B)=0$ for all $i \neq 0$ is shown using the isomorphism

$$
\rho_{\ell}\left({ }^{\mathrm{m}} \mathrm{H}^{i}(A \star B)\right)={ }^{p} \mathrm{H}^{i}\left(\rho_{\ell}(A \star B)\right)={ }^{p} \mathrm{H}^{i}\left(\rho_{\ell}(A) \star \ell \rho_{\ell}(B)\right) .
$$

For the classical $\ell$-adic convolution functor $\star \ell$ and the rightmost isomorphism see around Proposition 3.14. We now use that at least over a separably closed base field the convolution product of perverse equivariant sheaves is again perverse [57, Thm. 2.1]. We then conclude using the conservativity of the composite $\rho_{\ell, \bar{s}}$, cf. Synopsis 5.1 (vi).

The following proposition is a subtle part of the geometric Satake equivalence in the different settings $[7,8,30,52,57,65]$. Here we benefit from the existence of the symmetric monoidal structure in these settings and the faithfulness of the $\ell$-adic realization in Synopsis 5.1 (vi) to check the required compatibilities between the commutativity and associativity constraints.

Proposition 5.9 Let A, B, C $\in \operatorname{MTM}\left(L^{+} G \backslash L G / L^{+} G\right)$. There exist functorial equivalences

$$
c_{A, B}: A \star B \simeq B \star A, \quad \text { and } \quad a_{A, B, C}:(A \star B) \star C \simeq A \star(B \star C),
$$

called commutativity and associativity constraints which are uniquely determined by the following two properties:

(i) The isomorphisms are colimit-preserving in each argument.

(ii) For any geometric point $\bar{s} \rightarrow S$, the constraints map under the composition of functors (Corollary 5.7, Synopsis 5.1 (vi))

$$
\operatorname{MTM}\left(L^{+} G \backslash L G / L^{+} G\right)^{c} \stackrel{\simeq}{\longrightarrow} \operatorname{MTM}(\mathrm{Gr})^{c} \stackrel{\rho_{\ell, \bar{s}}}{\longrightarrow} \operatorname{Perv}\left(\mathrm{Gr}_{\bar{s}}, \mathbf{Q}_{\ell}\right)
$$

to the usual constraints used in geometric Satake as, e.g., in [65, Prop. 2.21].

In particular, the category $\operatorname{MTM}\left(L^{+} G \backslash L G / L^{+} G\right) \simeq \operatorname{MTM}(\mathrm{Gr})$ is a symmetric monoidal tensor category with respect to these constraints.

Proof Uniqueness. By property (i) it is enough to characterize the constraints on the subcategory of compact objects. For any $\bar{s} \rightarrow S$ as above, the functor 
$\rho_{\ell, \bar{s}}: \operatorname{MTM}\left(L^{+} G \backslash L G / L^{+} G\right)^{c} \rightarrow \operatorname{Perv}\left(\mathrm{Gr}_{\bar{s}}, \mathbf{Q}_{\ell}\right)$ is faithful by Synopsis 5.1 (vi). This implies uniqueness.

Existence. Note that once $c_{A, B}, a_{A, B, C}$ with properties (i) and (ii) exist, these constraints have to satisfy the axioms required for a symmetric monoidal category (hexagon axiom etc.). This follows from the corresponding identities for the $\ell$-adic Satake equivalence, and the faithfulness of the functor in (ii). It remains to construct the constraints. The associativity constraint was constructed in Lemma 3.7. For the commutativity constraint, we use the categorical analogue of Gelfand's trick whose construction is explained in $[8, \S 5.3 .8]$ and $[65, \S 2.4 .3]$. The use of prestacks simplifies the construction a little bit: Fix a pinning $(G, B, T, X)$. Define the anti-involution $\theta: G \rightarrow G, g \mapsto\left(g^{*}\right)^{-1}=\left(g^{-1}\right)^{*}$ where $(-)^{*}$ denotes the Cartan involution. The latter is characterized by the fact that it maps a dominant cocharacter $\lambda \in X_{*}(T)^{+}$ to $-w_{0} \lambda$, where $w_{0}$ is the longest element in the finite Weyl group. By functoriality, we obtain an anti-involution on $L:=L G$ preserving $L^{+}:=L^{+} G$, and thus an equivalence of prestacks, still denoted by

$$
\theta: L^{+} \backslash L / L^{+} \stackrel{\simeq}{\longrightarrow} L^{+} \backslash L / L^{+}
$$

For all $A, B \in \operatorname{MTM}\left(L^{+} G \backslash L G / L^{+} G\right)$ we construct a canonical isomorphism $\theta^{!}(A \star B) \simeq\left(\theta^{!} B\right) \star\left(\theta^{!} A\right)$ as follows: there is a (homotopy) Cartesian diagram of prestacks

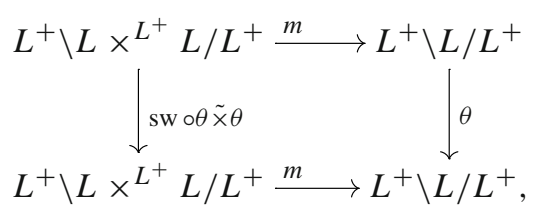

where sw is induced from the switch $L \times L \rightarrow L \times L,\left(g_{1}, g_{1}\right) \mapsto\left(g_{2}, g_{1}\right)$. Hence, we obtain

$$
\begin{aligned}
\theta^{!}(A \star B) & =\theta^{!} m_{!} p^{!}(A \otimes B)=m_{!}(\mathrm{sw} \circ \theta \tilde{\times} \theta)^{!} p^{!}(A \otimes B) \\
& =m_{!}(\theta \tilde{\times} \theta)^{!} p^{!}(B \otimes A) \stackrel{(*)}{=} m_{!} p^{!}\left(\theta^{!} B \otimes \theta^{!} A\right)=\left(\theta^{!} B\right) \star\left(\theta^{!} A\right) .
\end{aligned}
$$

The isomorphism labelled (*) follows from $(\theta \times \theta) !(A \otimes B)=\left(\theta^{!} A\right) \otimes\left(\theta^{!} B\right)$, which holds since $\theta$ is a placid map and $\mathrm{DM}^{!}$is symmetric lax monoidal as a functor on placid prestacks with placid maps. Next we define an isomorphism of (plain) endofunctors on $\operatorname{MTM}\left(L^{+} G \backslash L G / L^{+} G\right)$ denoted by

$$
e: \theta^{!} \stackrel{\simeq}{\longrightarrow} \mathrm{id}
$$

For this we fix a square root $i \in \mathbf{C}$ of -1 , and work temporarily with coefficients in $\mathbf{Q}(i)$. We define $e$ on each indecomposable object $\mathrm{IC}_{L, \mu}$ (Corollary 5.6) to be the map 
corresponding to $i^{(2 \rho, \mu)} \cdot \mathrm{id}$ under

$$
\operatorname{Hom}_{\mathbf{Q}(i)}\left(\theta ! \mathrm{IC}_{L, \mu}, \operatorname{IC}_{L, \mu}\right)=\operatorname{Hom}_{\mathbf{Q}(i)}\left(L\left[d_{\mu}\right], L\left[d_{\mu}\right]\right),
$$

obtained by restriction to the open orbit $L^{+} G \backslash L G^{\mu} / L^{+} G \subset L^{+} G \backslash L G^{\leq \mu} / L^{+} G$ (this orbit is invariant under $\theta$ ). Since $i^{(2 \rho, \mu)}$. id is a central endomorphism, one checks that $e$ is functorial. We leave the details to the reader.

Next define

$$
c_{A \star B}^{\prime}: A \star B \stackrel{e_{A \star B}}{\longleftarrow} \theta^{!}(A \star B) \simeq\left(\theta^{!} B\right) \star\left(\theta^{!} A\right) \stackrel{e_{B} \star e_{A}}{\longrightarrow} B \star A,
$$

which is invariant under the Galois automorphism $i \mapsto-i$, and thus defined over $\mathbf{Q}$. Finally, if both objects $A, B$ have constant parity, we define $c_{A \star B}:=(-1)^{p(A) p(B)} c_{A \star B}^{\prime}$ where $p$ denotes the parity function from (5.2). For general objects $A, B$, not necessarily of constant parity, we use Corollary 5.3 (i) to extend $c_{A \star B}$ linearly. See also [8, 5.3.21] and [52, Rmk. $6.2 \mathrm{ff}$.] for slicker formulations. It is a difficult theorem which is proven in [65, Prop. 2.21], relying on [51], that the $\ell$-adic realization $\rho_{\ell}\left(c_{A \star B}\right)$ is the (modified) commutativity constraint coming from the fusion interpretation of the convolution product. This finishes the construction of the constraints.

Remark 5.10 If $S$ is the spectrum of a field, then the above commutativity constraint can also be constructed as e.g. in [8] and [52] (see also [26]) by using the fusion interpretation of the convolution product and the motivic nearby cycles functor constructed in $[4],[5, \S 10]$.

\subsection{Tannakian structure}

In this section, we show that the category $\operatorname{MTM}\left(L^{+} G \backslash L G / L^{+} G\right) \simeq \operatorname{MTM}(\mathrm{Gr})$, which admits a symmetric monoidal structure with respect to the convolution product $\star$ by Proposition 5.9, has in fact a Tannakian structure with fibre functor being the global motivic cohomology functor.

\subsubsection{The fiber functor}

The fiber functor is a motivic analogue of the augmentation map for the spherical Hecke algebra.

Definition 5.11 The fiber functor is the composition

$$
\begin{aligned}
& \omega: \operatorname{MTM}\left(L^{+} G \backslash L G / L^{+} G\right) \stackrel{\sigma^{!}}{\longrightarrow} \operatorname{MTM}(\mathrm{Gr}) \stackrel{\epsilon !}{\longrightarrow} \operatorname{DTM}(S) \\
& \stackrel{\mathrm{gr}^{\mathrm{rl}}}{\longrightarrow} \operatorname{MTM}(S) \stackrel{\mathrm{gr}^{\mathrm{W}}}{\longrightarrow} \operatorname{MTM}(S)^{\mathrm{w}=0}=\operatorname{Vect}_{\mathbf{Q}}
\end{aligned}
$$

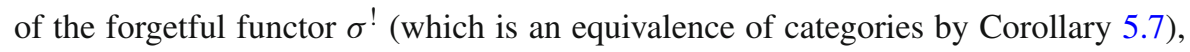
the pushforward along the structural map $\epsilon: \mathrm{Gr} \rightarrow S$ (which preserves Tate motives by [60, Lem. 3.1.19], using that the stratification of Gr by $L^{+} G$-orbits is cellular), 
followed by the grading functors for the classical motivic (which agrees in this case with the perverse motivic) $t$-structure and the weight structure (Corollary 4.2):

$$
\begin{aligned}
\mathrm{gr}^{\mathrm{cl}}: M \mapsto \bigoplus_{i}{ }^{\mathrm{cl}} \mathrm{H}^{i}(M), \\
\mathrm{gr}^{\mathrm{W}}: M \mapsto \bigoplus_{i}\left(\mathrm{gr}_{2 i}^{W} M\right)(i),
\end{aligned}
$$

and finally the equivalence [47] of pure Tate motives of weight 0 with the category of $\mathbf{Q}$-vector spaces (here we use that $S$ is connected).

As a consequence of the Kazhdan-Lusztig parity vanishing one obtains:

Corollary 5.12 Let $A \in \operatorname{MTM}\left(L^{+} G \backslash L G / L^{+} G\right)$ be of constant parity $p(A) \in \mathbf{Z} / 2$ (Corollary 5.3). Then ${ }^{\mathrm{cl}} \mathrm{H}^{i}\left(\epsilon ! \sigma^{!} A\right)=0$ whenever $i \not \equiv p(A) \bmod 2$.

Proof This is immediate from the conservativity of the $\ell$-adic realization as in Synopsis 5.1 (vi) using the well-known statement for $\ell$-adic sheaves. Here is an argument by reduction to Corollary 5.3 according to which $A$ has only, say, even classical cohomology, i.e., ${ }^{\mathrm{cl}} \mathrm{H}^{i}(A)=0$ for $i$ odd.

As in the proof of Corollary 5.3, we may assume that $A$ is compact. The functors $\left(\iota_{\mu}\right)$ ! and $\left(\iota_{\mu}\right)^{*}$ between DTM $\left(\mathrm{Gr}_{G}\right)$ and DTM $\left(\mathrm{Gr}_{G}^{\mu}\right)$ are exact with respect to the classical motivic t-structure since the corresponding statement is true for $\ell$-adic sheaves. Moreover, by localization, $\sigma^{!} A$ is an iterated extension of the $A_{\mu}:=\left(\iota_{\mu}\right) !\left(\iota_{\mu}\right)^{*} \sigma^{!} A$ where $\mu$ runs over the finitely many dominant cocharacters in the support of $A$. Using the long exact cohomology sequence for the ${ }^{\mathrm{cl}} \mathrm{H}$-cohomologies of $\epsilon ! \sigma^{!} A$, we may thus replace $A$ by $A_{\mu}$. We now use that the Iwahori stratification of $\mathrm{Gr}^{\mu}$ consists of affine spaces. By the same localization argument, we may replace $\mathrm{Gr}^{\mu}$ by such a stratum $\mathbf{A}_{S}^{n}$. We are left to showing that for the structural map $\mathbf{A}_{S}^{n} \stackrel{p}{\rightarrow} S, p_{!}: \operatorname{DTM}\left(\mathbf{A}_{S}^{n}\right) \rightarrow \operatorname{DTM}(S)$ preserves parity vanishing. In fact $p$ ! is an equivalence of categories, being the adjoint of $p^{!}: \operatorname{DTM}(S) \rightarrow \operatorname{DTM}\left(\mathbf{A}_{S}^{n}\right)$ which is fully faithful by homotopy invariance of DM and essentially surjective by definition of DTM.

It is a classical fact that the augmentation map from parahoric Hecke algebras to the coefficient field respects the multiplicative structure. The corresponding fact is also well-known in a categorified situation. The proof below is thus similar to, say, [65, Prop. 2.20].

Proposition 5.13 Let $\mathbf{f}$ be a facet and $\mathcal{P}:=\mathcal{P}_{\mathbf{f}}$ its associated parahoric subgroup of $L G$. Then the pull-push along the correspondence

$$
\mathcal{P} \backslash L G / \mathcal{P} \stackrel{\sigma}{\longleftarrow} L G / \mathcal{P} \stackrel{\epsilon}{\longrightarrow} S
$$

yields a functor

$$
\omega: \operatorname{DM}(\mathcal{P} \backslash L G / \mathcal{P}) \stackrel{\sigma^{!}}{\longrightarrow} \operatorname{DM}(L G / \mathcal{P}) \stackrel{\epsilon_{!}}{\longrightarrow} \operatorname{DM}(S)
$$


At least on the level of homotopy categories, this functor has a natural monoidal structure with respect to the convolution product on $\operatorname{DM}(\mathcal{P} \backslash L G / \mathcal{P})$ and the ordinary tensor product on $\mathrm{DM}(S)$.

Proof The left adjoint $\epsilon$ ! of $\epsilon$ ! exists since the étale sheafification $\mathrm{Fl}:=\mathrm{Fl}_{\mathbf{f}}=$ $(L G / \mathcal{P})^{\text {et }}$ is an ind-scheme, so the pushforward along the structural map to $S$ exists by [60, Thm. 2.4.2]. The unitality of $\omega$ is clear, since the monoidal unit is the skyscraper motive supported at the base point of Fl. To show the monoidality of $\omega$, we abbreviate $\tilde{X}:=L G \times L G / \mathcal{P}$ (all products are products of prestacks over $S$ ) and $X:=\mathcal{P} \backslash L G \times L G / \mathcal{P}=\mathcal{P} \backslash \tilde{X}$. The group $\mathcal{P}^{\text {op }} \times \mathcal{P}$ acts on $\tilde{X}$ (and $X$ ) by acting on the right on the first, and on the left on the second factor. We write $\Delta:=\left\{\left(p^{-1}, p\right) \mid p \in \mathcal{P}\right\} \subset \mathcal{P}^{\mathrm{op}} \times \mathcal{P}$ for the "diagonal" subgroup. The structural map $X \rightarrow S$ gives a map $\Delta \backslash X \rightarrow \Delta \backslash S$. On the other hand, the composition of the multiplication and structural map $L G / \mathcal{P} \stackrel{\epsilon}{\rightarrow} S$ yields another map

$$
\Delta \backslash X \stackrel{m}{\rightarrow} \mathcal{P} \backslash L G / \mathcal{P} \stackrel{\epsilon^{\prime}}{\rightarrow} \mathcal{P} \backslash S
$$

These two maps agree: this can be checked after precomposing with the epimorphism $\tilde{X} \rightarrow(\mathcal{P} \times \Delta) \backslash \tilde{X}=\Delta \backslash X$, where it boils down to using that the structural map $\tilde{X} \rightarrow S$ agrees with $\tilde{X} \stackrel{m}{\rightarrow} L G / \mathcal{P} \stackrel{\epsilon}{\rightarrow} S$, since $S$ is is the final object, and in particular is acted upon trivially by all copies of $\mathcal{P}$.

This shows that the following diagram of prestacks is cartesian, as soon as we omit the dotted map. Note that the map $\epsilon^{\prime} \times \epsilon^{\prime}$ arises as $\left(\mathcal{P}^{\text {op }} \times \mathcal{P}\right) \backslash(X \rightarrow S)$. Once we do include the dotted map, the small bottom left square is still cartesian (but the top left square does not commute):

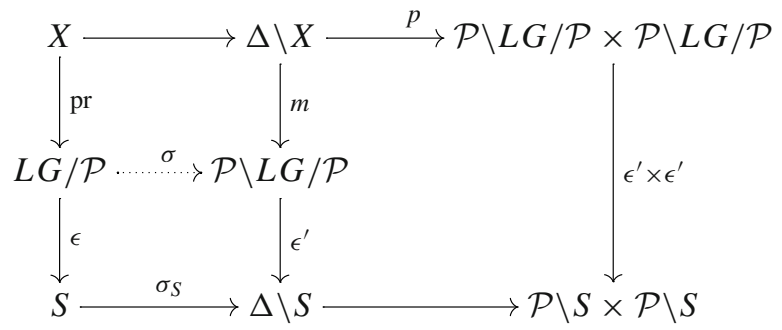

We then have $\omega(A \star B)=\epsilon ! \sigma ! m_{!} p^{!}(A \otimes B)$. As was already noted in the proof of Lemma 3.11, $\epsilon$ ! exchanges with the forgetful functor $\sigma^{!}$by ind-proper base change. Similarly, $\sigma_{S}^{!}$exchanges with $\left(\epsilon^{\prime} \circ m\right)$ ! , so the above is equivalent to $(\epsilon \times \epsilon)$ ! $(\sigma \times$ $\sigma)^{!}(A \otimes B)$. The !-pullback along the map $X \rightarrow\left(\mathcal{P}^{\text {op }} \times \mathcal{P}\right) \backslash X$ commutes with $\otimes$ by construction of $\otimes$, see Remark 3.2. Furthermore, $\otimes$ also commutes with the !-pushforward along the structural map $X \rightarrow S$. After reducing this claim to the case of finite-type $S$-schemes (instead of the ind-finite type ind-scheme $X$ ), this is a consequence of the projection formula. Hence the above object is equivalent to $\omega(A) \otimes \omega(B)$. 
Theorem 5.14 The category of compact objects $\operatorname{MTM}\left(L^{+} G \backslash L G / L^{+} G\right)^{\mathrm{c}}=\operatorname{MTM}(\mathrm{Gr})^{c}$ (Corollary 5.7), endowed with the convolution product, the constraints from Proposition 5.9 and $\omega$ as fibre functor is a neutral Tannakian category over $\mathbf{Q}$ ([21, Ch. II, Def. 2.19]).

Proof We check the conditions in [21, Ch. II, Prop. 1.20] using the functor $\omega$ (Definition 5.11).

(i) The functor whas a monoidal structure. By Proposition 5.13, it remains to observe that $\mathrm{gr}^{\text {perv }}$ and $\mathrm{gr}^{\mathrm{W}}$ have monoidal structures: both functors and the respective tensor products preserve colimits, so we may consider the subcategories of compact objects instead. Then we are in the situation of [47]: the characterization of $\operatorname{DTM}(S)^{\leq 0, \mathrm{c}}$ (resp. $\left.\operatorname{DTM}(S)^{\geq 1, \mathrm{c}}\right)$ as the subcategories generated under extensions by the objects $1_{S}(n)[m]$ with $n \in \mathbf{Z}$ and $m \geq 0$ (resp. $m \leq-1$ ) immediately shows the monoidality of $\mathrm{gr}^{\mathrm{cl}}$ restricted to the subcategory of complexes concentrated in either even or odd degrees. Now we take the parity vanishing of Corollary 5.12 into account. The monoidality of $\mathrm{gr}^{\mathrm{W}}$ holds by [47, Thm. 1.4.v].

(ii) The functor $\omega$ is $\mathbf{Q}$-linear, exact and faithful. The functor $\omega$ is clearly $\mathbf{Q}$-linear. To check the exactness, suppose

$$
0 \rightarrow M^{\prime} \rightarrow M \rightarrow M^{\prime \prime} \rightarrow 0
$$

is a short exact sequence in $\operatorname{MTM}\left(L^{+} G \backslash L G / L^{+} G\right)=\operatorname{MTM}(\mathrm{Gr})$. To show it maps to an exact sequence under $\omega$, we may assume $M^{\prime}$ and $M^{\prime \prime}$ are indecomposable (since $\operatorname{Ext}_{\mathrm{MTM}(\mathrm{Gr})}^{1}(-,-)$ commutes with finite direct sums in each variable), i.e., by Corollary 5.6 of the form $M^{\prime}=\mathrm{IC}_{L^{\prime}, \mu^{\prime}}, M^{\prime \prime}=\mathrm{IC}_{L^{\prime \prime}, \mu^{\prime \prime}}$ for some $\mu^{\prime}, \mu^{\prime \prime} \in X_{*}(T)^{+}$and some indecomposable motives $L^{\prime}, L^{\prime \prime} \in \operatorname{MTM}(S)$. By Corollary 5.5, the extension splits unless $\mu^{\prime}=\mu^{\prime \prime}=: \mu$ in which case $M \simeq \mathrm{IC}_{L, \mu}$, where $L$ is an extension in $\operatorname{MTM}(S)$ of $L^{\prime}$ and $L^{\prime \prime}$. We conclude that $\omega$ is exact since $\omega\left(\mathrm{IC}_{L, \mu}\right) \simeq \omega\left(L \star \mathrm{IC}_{1, \mu}\right) \simeq \omega(L) \otimes \omega\left(\mathrm{IC}_{1, \mu}\right)$ using (i) above. The faithfulness of $\omega$ follows from the conservativity of $\omega$, which in its turn follows from the conservativity of the $\ell$-adic realization at some geometric point $\bar{s} \rightarrow S$ (Synopsis 5.1 (vi)) and the conservativity of the fiber functor in the $\ell$-adic situation.

(iii) The constraints constructed in Proposition 5.9 give the usual constraints in Vect $\mathbf{Q}$ after applying $\omega$. This is immediate from the $\ell$-adic case, see [65, Prop. 2.21] using that the realization is faithful. We stress that one needs to change the natural commutativity constraints one has on complexes: this is possible by the parity vanishing, see also the passage from $c_{A \star B}^{\prime}$ to $c_{A \star B}$ in the proof of Proposition 5.9.

(iv) Neutral object. The skyscraper at the base point $\operatorname{IC}_{0} \in \operatorname{MTM}\left(L^{+} G \backslash L G / L^{+} G\right)$ satisfies $\operatorname{End}\left(\mathrm{IC}_{0}\right)=\mathbf{Q}$ and $\omega\left(\mathrm{IC}_{0}\right)$ is 1-dimensional.

(v) Any $M \in \operatorname{MTM}\left(L^{+} G \backslash L G / L^{+} G\right)$ with $\operatorname{dim}_{\mathbf{Q}} \omega(M)=1$ admits a dual object $M^{-1}$ such that $M \star M^{-1}=\mathrm{IC}_{0}$. If $\operatorname{dim}_{\mathbf{Q}} \omega(M)=1$, then $M$ is indecomposable by the faithfulness of $\omega$, i.e., $M \simeq \mathrm{IC}_{L, \mu}$ with $L$ indecomposable. Since $\omega\left(\mathrm{IC}_{L, \mu}\right)=$ $\omega(L) \otimes \omega\left(\mathrm{IC}_{1, \mu}\right)$, the motive $L \in \operatorname{MTM}(S)$ is also pure, and hence $\otimes$-invertible. Moreover, $\operatorname{dim}_{\mathbf{Q}} \omega\left(\mathrm{IC}_{\mu}\right)=\operatorname{dim}_{\mathbf{Q}_{\ell}} \omega_{\ell}\left(\mathrm{IC}_{\mu, \ell}\right)$, where the subscripts denote the corresponding functors in the $\ell$-adic realization. By the $\ell$-adic Satake equivalence 
(see e.g. [7, §9] $]^{2}$, or [57, Cor. 3.5]), this implies $\operatorname{dim}(\mathrm{Gr} \leq \mu / S)=0$, i.e., $\mathrm{IC}_{\mu}$ is dualizable with respect to $\star$. Namely its dual is $\mathrm{IC}_{\mu} \star \mathrm{IC}_{-\mu} \simeq \mathrm{IC}_{0}$.

\section{The dual group}

In this final section we determine the Tannaka dual of the categories $\operatorname{MTM}\left(L^{+} G_{S} \backslash L G_{S} / L^{+} G_{S}\right)$ and the so-called Satake category

$$
\operatorname{Sat}_{G, S} \subset \operatorname{MTM}\left(L^{+} G_{S} \backslash L G_{S} / L^{+} G_{S}\right),
$$

which can be thought of as the semi-simplification of the latter category. We show in Theorem 6.8 that the Tannaka dual of Sat ${ }_{G, S}$ is Deligne's modified Langlands dual group $\widehat{G}_{1} / \mathbf{Q}$ as constructed in $[24, \S 2]$. In particular, this group is independent of the (connected) base scheme $S$.

For $S=\operatorname{Spec} \mathbf{F}_{q}$, the inclusion (6.1) is an equivalence. For more general bases $S$, we show in Theorem 6.14 that the Tannakian group of $\operatorname{MTM}\left(L^{+} G_{S} \backslash L G_{S} / L^{+} G_{S}\right)$ is the semi-direct product of $\widehat{G}_{1}$ with a pro-unipotent affine group scheme coming from extensions between Tate motives on $S$.

Throughout Sect. 6, the base scheme $S$ is as in Notation 2.1. Also recall Synopsis 5.1.

\subsection{The Satake category}

Definition 6.2 The Satake category $\mathrm{Sat}_{G}=\mathrm{Sat}_{G, S}$ is the full subcategory of $\operatorname{MTM}\left(L^{+} G \backslash L G / L^{+} G\right)$ generated by means of arbitrary direct sums (as opposed to allowing extensions) by the intersection motives $\operatorname{IC}_{\mu}(n), \mu \in X_{*}(T)^{+}, n \in \mathbf{Z}$.

Lemma 6.3 For $L, L^{\prime} \in \operatorname{MTM}(S)$ and $\lambda, \mu \in X_{*}(T)^{+}$, we have natural identifications

$$
\operatorname{Hom}_{M T M\left(G_{G}\right)}\left(\operatorname{IC}_{L, \lambda}, \mathrm{IC}_{L^{\prime}, \mu}\right)= \begin{cases}\operatorname{Hom}_{\mathrm{MTM}(S)}\left(L, L^{\prime}\right) & \lambda=\mu \\ 0 & \lambda \neq \mu\end{cases}
$$

Proof For $\lambda=\mu$, this is a standard property of intermediate extensions, see [44, Cor. III.5.11]. To show the vanishing in case $\lambda \neq \mu$, we may assume $L$ is a simple object of $\operatorname{MTM}(S)$. In this case, $\mathrm{IC}_{L, \mu}$ is also simple, so any non-zero morphism would need to be an isomorphism, which is impossible if $\lambda \neq \mu$.

Corollary 6.4 The category $\mathrm{Sat}_{G}$ is abelian. Its subcategory of compact objects $\mathrm{Sat}_{G}^{\mathrm{c}}$ is semi-simple.

\footnotetext{
2 The reference uses constructible sheaves in the analytic topology over $\mathbf{C}$, but the same argument works by invoking [35, Cor. 6.9]. 
Lemma 6.5 The full subcategory $\operatorname{Sat}_{G} \subset \operatorname{MTM}\left(L^{+} G \backslash L G / L^{+} G\right)$ is stable under the convolution product.

Proof We have to show that $M:=\mathrm{IC}_{\mu} \star \mathrm{IC}_{\lambda}$ is a direct sum of some intersection motives of the form $\operatorname{IC}_{\kappa}(n)$. (A priori we only know it is a successive extension of twists of some $\mathrm{IC}_{\kappa}$.) By Corollary 5.6, $M=\oplus_{(L, \mu)} \mathrm{IC}_{L, \mu}$ with $L$ indecomposable. The intersection motives $\mathrm{IC}_{\mu}$ and $\mathrm{IC}_{\lambda}$ are pure by Theorem 4.1, hence so is $M$ by Proposition 4.8. Therefore, each direct summand $\mathrm{IC}_{L, \mu}$ is also pure. Let $j: \mathrm{Gr}_{\mu} \rightarrow \mathrm{Gr} \leq \mu$ be the open stratum. Since $j^{*}=j^{!}$, the motive $j^{*} \mathrm{IC}_{L, \mu}=L\left[d_{\mu}\right]$ is also pure, which implies $L$ is pure. Since $L$ is also indecomposable, it is of the form $L=1_{S}(n)$ for some $n \in \mathbf{Z}$, hence $\operatorname{IC}_{L, \mu}=\operatorname{IC}_{\mu}(n) \in \operatorname{Sat}_{G}$.

Corollary 6.6 The subcategory $\mathrm{Sat}_{G}^{\mathrm{c}} \subset \mathrm{Sat}_{G}$ spanned by the compact objects in the Satake category has the following properties:

(i) $\mathrm{Sat}_{G}^{\mathrm{c}}$ is a neutral Tannakian subcategory.

(ii) For any map $f: T \rightarrow S$ of connected schemes as in Notation 2.1, there is an equivalence of neutral Tannakian categories

$$
f^{*}: \operatorname{Sat}_{G, S}^{c} \stackrel{\simeq}{\longrightarrow} \operatorname{Sat}_{G, T}^{c}
$$

having the property $f^{*} \mathrm{IC}_{\mu, S}(n)=\operatorname{IC}_{\mu, T}(n)$ for all $\mu \in X_{*}(T)^{+}, n \in \mathbf{Z}$.

Proof Part (i) is immediate from Lemma 6.5 and Proposition 5.14. For (ii), we use Theorem 2.15 which gives $f^{*} \operatorname{IC}_{\mu, S}(n)=\operatorname{IC}_{\mu, T}(n)$, so that $f^{*}$ is an equivalence of Q-linear abelian categories. The compatibility of $f^{*}$ with the convolution product was checked in Lemma 3.15. Also $\omega\left(\operatorname{IC}_{\mu, S}(n)\right)=\omega\left(\operatorname{IC}_{\mu, T}(n)\right)$ is immediate from Definition 5.11. The rest is clear from the characterization of the constraints in (5.9).

Now fix a pinning $(G, B, T, X)$, and denote by $(\widehat{G}, \widehat{B}, \widehat{T}, \widehat{X})$ the dual group in the sense of Langlands formed over $\mathbf{Q}$. By definition $\widehat{G}$ is a split reductive $\mathbf{Q}$-group with split maximal torus $\widehat{T}$, and Borel subgroup $\widehat{B}$. Denote by $\widehat{T}_{\text {ad }}$ the image of $\widehat{T}$ under the map $\widehat{G} \rightarrow \widehat{G}_{\text {ad }}$ to the adjoint group. Then we may view the half sum $\rho$ of the roots in $B$ (=coroots in $\widehat{B}$ ) as a cocharacter $\rho: \mathbf{G}_{m, \mathbf{Q}} \rightarrow \widehat{T}_{\mathrm{ad}} \subset \widehat{G}_{\mathrm{ad}}$. We let $\mathbf{G}_{m, \mathbf{Q}}$ act through $\rho$ by inner automorphisms on $(\widehat{G}, \widehat{B}, \widehat{T})$ from the right. Colloquially speaking, this action is given by the formula $g \cdot \lambda=\rho(\lambda)^{-1} g \rho(\lambda)$. We consider the semi-direct product $\widehat{G}_{1}:=\widehat{G} \rtimes \mathbf{G}_{m}, \mathbf{Q}$ which is again a split reductive $\mathbf{Q}$-group with Borel pair $\widehat{T} \times \mathbf{G}_{m, \mathbf{Q}}=: \widehat{T}_{1} \subset \widehat{B}_{1}:=\widehat{B} \rtimes \mathbf{G}_{m, \mathbf{Q}}$.

For each $\mu \in X_{*}(T)^{+}$, and $n \in \mathbf{Z}$ we get an irreducible algebraic $\widehat{G}_{1}$-representation [39, Ch. II.5]

$$
V_{\mu}(n) \stackrel{\text { def }}{=} \operatorname{Ind}_{\widehat{B}_{1}^{\text {op }}}^{\widehat{G}_{1}}\left(\mu_{n}\right)
$$

where $\widehat{B}_{1}^{\text {op }} \subset \widehat{G}_{1}$ denotes the Borel opposite to $\widehat{B}_{1}$, and $\mu_{n}: \widehat{B}_{1}^{\text {op }} \rightarrow \widehat{T}_{1} \rightarrow \mathbf{G}_{m, \mathbf{Q}}$ is the composition of the projection with the character $(\mu, n) \in X_{*}(T)^{+} \times \mathbf{Z}=$ $X^{*}\left(\widehat{T}_{1}\right)^{+}$. Then $V_{\mu}(n)$ is the representation of $\widehat{G}_{1}$ of highest weight $(\mu, n)$. We denote 
by $\operatorname{Rep}_{\mathbf{Q}}^{\mathrm{fd}}\left(\widehat{G}_{1}\right)$ the category of algebraic $\widehat{G}_{1}$-representations on finite-dimensional Qvector spaces. This category is semi-simple with simple objects the highest weight representations as above.

Remark 6.7 The split reductive group $\widehat{G}_{1}$ is Deligne's modified Langlands dual group constructed in [24], see also [20]. More precisely, one checks that the map $(g, \lambda) \mapsto$ $\left(g \cdot(2 \rho)(\lambda), \lambda^{2}\right)$ induces a short exact sequence of Q-group schemes

$$
1 \rightarrow \boldsymbol{\mu}_{2} \rightarrow \widehat{G} \times \mathbf{G}_{m, \mathbf{Q}} \rightarrow \widehat{G}_{1} \rightarrow 1
$$

where $\boldsymbol{\mu}_{2} \simeq \mathbf{Z} / 2$ is the constant subgroup scheme generated by the element $(\epsilon,-1)$, $\epsilon:=(2 \rho)(-1)$. It follows that the semi-direct product $\widehat{G}_{1}=\widehat{G} \rtimes \mathbf{G}_{m, \mathbf{Q}}$ is (canonically) a direct product if $\epsilon=1$. The latter condition is also equivalent to $\rho$ being a cocharacter of $\widehat{T}$ (as opposed to $\widehat{T}_{\text {ad }}$ ). For example, this is the case if $G$ is simply connected, so that $\widehat{G}_{\mathrm{ad}}=\widehat{G}$ is adjoint. We note that the difference of $\widehat{G}$ versus $\widehat{G}_{1}$ relates to the notions of $L$-algebraic versus $C$-algebraic as introduced by Buzzard and Gee in [13]. For further discussion and examples we refer to [13, Prop. $5.39 \mathrm{ff}$.].

Theorem 6.8 There is an equivalence of Tannakian categories

$$
\left(\operatorname{Sat}_{G}^{\mathrm{c}}, \star, \omega\right) \simeq\left(\operatorname{Rep}_{\mathbf{Q}}^{\mathrm{fd}}\left(\widehat{G}_{1}\right), \otimes, v\right),
$$

where $v: \operatorname{Rep}_{\mathbf{Q}}^{\mathrm{fd}}\left(\widehat{G}_{1}\right) \rightarrow \operatorname{Vec}_{\mathbf{Q}}$ denotes the forgetful functor. The intersection motives $\mathrm{IC}_{\mu}(n)$ correspond to the irreducible $\widehat{G}_{1}$-representations $V_{\mu}(n)$ for $(\mu, n) \in$ $X_{*}(T)^{+} \times \mathbf{Z}=X^{*}\left(\widehat{T}_{1}\right)^{+}$.

Proof We denote by $\operatorname{Aut}_{\mathrm{Sat}_{G}}^{\star}(\omega)$ the affine Q-group scheme of tensor automorphisms of $\omega$ provided by the neutral Tannakian category $\left(\right.$ Sat $\left._{G}^{c}, \star, \omega\right)$, cf. [21, Ch. II, Thm. 2.11]. The Satake category $\mathrm{Sat}_{G}=\mathrm{Sat}_{G, S}$ is independent from the (connected) base scheme $S$ by Corollary 6.6 (ii), i.e., for any map $T \rightarrow S$ of schemes as in Notation 2.1 we have

$$
\operatorname{Aut}_{\mathrm{Sat}_{G, S}^{\mathrm{c}}}^{\star}(\omega)=\operatorname{Aut}_{\mathrm{Sat}_{G, T}^{\mathrm{c}}}^{\mathrm{c}}(\omega)
$$

As $G$ is split, it is defined over $\mathbf{Z}$, and in particular over any scheme as is the affine Grassmannian. By Corollary 6.6, we may assume that $S=\operatorname{Spec}\left(\mathbf{F}_{p}\right)$ is a finite field, and we denote $\widetilde{G}_{1}:=\operatorname{Aut}_{\mathrm{Sat}_{G}^{\mathrm{c}}}^{\star}(\omega)$. For any $\ell \neq p$, consider the $\ell$-adic realization

$$
\rho_{\ell}: \operatorname{Sat}_{G}^{\mathrm{c}} \rightarrow \operatorname{Perv}_{L^{+}}\left(\mathrm{Gr}, \mathbf{Q}_{\ell}\right)
$$

Let $\mathrm{Sat}_{G, \ell}^{\mathrm{c}}$ be the essential image of $\rho_{\ell}$, i.e., the full subcategory of $\operatorname{Perv}_{L^{+} G}\left(\mathrm{Gr}, \mathbf{Q}_{\ell}\right)$ consisting of the objects isomorphic to $\rho_{\ell}\left(\bigoplus_{i} \operatorname{IC}_{\mu_{i}}\left(n_{i}\right)\right)=\bigoplus_{i} \operatorname{IC}_{\ell, \mu_{i}}\left(n_{i}\right)$. Under the $\ell$-adic convolution product it is a Tannakian category; its fiber functor, denoted by $\omega_{\ell}$, is defined the same way as $\omega$. For any $M, N \in \mathrm{Sat}_{G, \ell}^{\mathrm{c}}$, Lemma 6.3 and analogous computations in the $\ell$-adic context give natural isomorphisms

$$
\operatorname{Hom}_{\mathrm{Sat}_{G}^{\mathrm{c}}}(M, N) \otimes_{\mathbf{Q}} \mathbf{Q}_{\ell}=\operatorname{Hom}_{\mathrm{Sat}_{G, \ell}^{\mathrm{c}}}\left(\rho_{\ell}(M), \rho_{\ell}(N)\right),
$$


so that

$$
\operatorname{Aut}_{S a a_{G}^{\mathrm{c}}}^{\star}(\omega) \otimes_{\mathbf{Q}} \mathbf{Q}_{\ell}=\operatorname{Aut}_{\mathrm{Sat}_{G, \ell}^{\mathrm{c}}}^{\mathrm{c}}\left(\omega_{\ell}\right)
$$

By the $\ell$-adic geometric Satake equivalence (in particular [37, Rmk. 2.10], [61, Prop. A.6], [66, 5.5.14]), we deduce an isomorphism

$$
\widetilde{G}_{1} \otimes_{\mathbf{Q}} \mathbf{Q}_{\ell} \simeq \widehat{G}_{1} \otimes_{\mathbf{Q}} \mathbf{Q}_{\ell}
$$

This holds for all prime numbers $\ell \neq p$. Since we have a zig zag $\mathbf{F}_{p} \leftarrow \mathbf{Z} \rightarrow \mathbf{F}_{p^{\prime}}$ for any pair of primes $p, p^{\prime}$, we see that (6.9) also holds for $\ell=p$. Using (6.9) for one prime number $\ell$, it follows that $\widetilde{G}_{1}$ is (geometrically) connected and reductive: we use fpqc descent for the extension $\mathbf{Q}_{\ell} / \mathbf{Q}$ for the properties '(geometrically) connected', 'of finite type' and 'smooth' [64, 04KV, 02KZ, 02VL], and further that the unipotent radical commutes with arbitrary field extensions over perfect fields for the property 'reductive' [17, Prop. 1.1.9]. If $\widetilde{G}_{1}$ is also split, then we have $\widetilde{G}_{1} \simeq \widehat{G}_{1}$ over $\mathbf{Q}$ by the isomorphism theorem ([18, Thm. 6.1.17]).

However, the condition (6.9) for all primes $\ell$ is not enough to ensure that $\widetilde{G}_{1}$ is split ([32]), and we argue as follows. By construction, the functor $\omega: \mathrm{Sat}_{G}^{\mathrm{c}} \rightarrow \operatorname{Vec}_{\mathbf{Q}}$ is equipped with a $\mathbf{Z}$-grading coming from the grading on ${ }^{\mathrm{cl}} \mathrm{H}^{*}$ in Definition 5.11. This defines a cocharacter $2 \widetilde{\rho}: \mathbf{G}_{m, \mathbf{Q}} \rightarrow \widetilde{G}_{1}$ via the Tannakian formalism, and we denote by $\widetilde{T}_{1} \subset \widetilde{G}_{1}$ its centralizer. Under the $\ell$-adic realization we have $2 \widetilde{\rho}=2 \rho$ by [66, 5.3.20]. Then, for all primes $\ell$, we have

$$
\widetilde{T}_{1} \otimes_{\mathbf{Q}} \mathbf{Q}_{\ell} \simeq \widehat{T}_{1} \otimes_{\mathbf{Q}} \mathbf{Q}_{\ell}
$$

compatible with the isomorphism (6.9). Using (6.10) for one prime, this implies as above that $\widetilde{T}_{1}$ is a commutative reductive group over $\mathbf{Q}$, and thus must be a torus. As $\widehat{T}_{1} \subset \widehat{G}_{1}$ is a maximal torus (6.10) implies that $\widetilde{T}_{1} \subset \widetilde{G}_{1}$ is a maximal torus (as this can be checked over the algebraically closed overfield $\left.\overline{\mathbf{Q}}_{\ell}\right)$. However, the $\operatorname{Gal}(\overline{\mathbf{Q}} / \mathbf{Q})$ Galois representation $X_{*}\left(\widetilde{T}_{1, \overline{\mathbf{Q}}}\right)$ is trivial at all primes $\ell$, and hence must be trivial by Minkowski's theorem. This shows that $\widetilde{T}_{1}$ is a maximal split torus of $\widetilde{G}_{1}$.

\subsection{The full Tannakian group}

Definition 6.11 Let $\widetilde{G}_{S}$ be the Tannaka dual group of the Tannakian category $\operatorname{MTM}\left(L^{+} G_{S} \backslash L G_{S} / L^{+} G_{S}\right)^{\mathrm{c}}$ (Theorem 5.14).

Example 6.12 For $S=\operatorname{Spec} \mathbf{F}_{q}$, the inclusion $\operatorname{Sat}_{G}^{\mathrm{c}} \rightarrow \operatorname{MTM}\left(L^{+} G \backslash L G / L^{+} G\right)^{\mathrm{c}}$ is an equivalence. This follows from Corollary 5.5, and the semisimplicity of $\operatorname{MTM}\left(\mathbf{F}_{q}\right)^{\mathrm{c}}$ (which follows from $\operatorname{Hom}_{\operatorname{DTM}\left(\mathbf{F}_{q}\right)}(1,1(i)[n])=0$ unless $n=i=0$ because the higher algebraic $K$-theory of finite fields is torsion by Quillen's computation [55]). Therefore

$$
\widetilde{G}_{\mathbf{F}_{q}}=\widehat{G}_{1} \text {. }
$$


We now exhibit the relation of the category $\operatorname{Sat}_{G}^{\mathrm{c}}$ and $\operatorname{MTM}\left(L^{+} G \backslash L G / L^{+} G\right)^{\mathrm{c}}=$ $\operatorname{MTM}\left(\mathrm{Gr}_{G}\right)^{\mathrm{c}}$ (Theorem 5.14) over other bases. In short, the category $\operatorname{MTM}\left(\mathrm{Gr}_{G}\right)^{\mathrm{c}}$ arises by amalgamating Tate motives on the base $S$ together with the intersection motives arising from the presence of the group $G$.

Recall from [47] that $\operatorname{MTM}(S)^{\mathrm{c}}$ is a neutral Tannakian category with fibre functor

$$
\operatorname{MTM}(S)^{\mathrm{c}} \stackrel{\mathrm{gr}^{\mathrm{W}}}{\rightarrow} \operatorname{MTM}(S)^{\mathrm{c}, \mathrm{W}=0}=\operatorname{Vect}_{\mathbf{Q}}
$$

which is the special case of Theorem 5.14 for the trivial group. We denote its Tannaka dual by $\mathscr{G}_{S}$. (It is the same as $\widetilde{G}_{S}$ for the trivial group $G=1$ ). The semi-simplification of $\operatorname{MTM}(S)^{\mathrm{c}}$ is just $\operatorname{MTM}(S)^{\text {pure,c }}$, which is equivalent to $\mathbf{Z}$-graded $\mathbf{Q}$-vector spaces. Equivalently, its Tannaka dual is $\mathbf{G}_{\mathrm{m}, \mathbf{Q}}$. Therefore $\mathscr{G}_{S}$ sits in a split exact sequence

$$
1 \rightarrow \mathscr{U}_{S} \rightarrow \mathscr{G}_{S} \rightarrow \mathbf{G}_{\mathrm{m}} \rightarrow 1
$$

where $\mathscr{U}_{S}$ is the pro-unipotent radical of $\mathscr{G}_{S}$. We have the following commutative diagram of neutral Tannakian categories

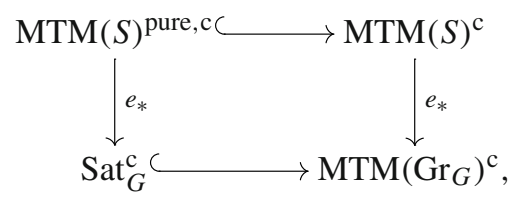

where $e: S \rightarrow \mathrm{Gr}_{G}$ is the inclusion of the base point. It induces a commutative diagram of the corresponding Tannaka dual groups

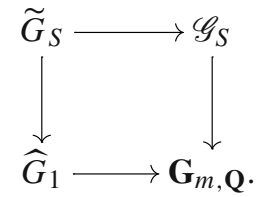

Theorem 6.14 The diagram (6.13) is Cartesian, i.e., it induces an isomorphism Qgroup schemes

$$
\alpha: \widetilde{G}_{S} \stackrel{\simeq}{\longrightarrow} \widehat{G}_{1} \times \mathbf{G}_{\mathrm{m}} \mathscr{G}_{S}=\mathscr{U}_{S} \rtimes \widehat{G}_{1} .
$$

In other words, there is an equivalence of Tannakian categories

$$
\operatorname{MTM}\left(L^{+} G \backslash L G / L^{+} G\right)^{\mathrm{c}}=\operatorname{MTM}\left(\operatorname{Gr}_{G}\right)^{\mathrm{c}}=\operatorname{Rep}_{\mathbf{Q}}^{\mathrm{fd}}\left(\mathscr{U}_{S} \rtimes \widehat{G}_{1}\right)
$$

between the category of compact mixed Tate motives on the double quotient $L^{+} G \backslash L G / L^{+} G$ over $S$ (or equivalently, compact mixed Tate motives on $\mathrm{Gr}_{G}$, cf. Theorem 5.14) and the category of finite-dimensional representations of $\mathscr{U}_{S} \rtimes \widehat{G}_{1}$ (regarded as a pro-algebraic group over $\mathbf{Q})$. 
Proof To check $\alpha$ is an isomorphism, we consider a $\mathbf{Q}$-algebra $R$ and evaluate the $R$-points of the two group schemes. Recall that by definition that $\widetilde{G}(R)$ consists of the tensor automorphisms of the fiber functor $\omega: \operatorname{MTM}\left(\mathrm{Gr}_{G}\right)^{\mathrm{c}} \rightarrow \operatorname{Vect}_{\mathbf{Q}}$, i.e., families of additive $R$-linear automorphisms $g_{X}: \omega(X) \otimes R \rightarrow \omega(X) \otimes R$ satisfying natural compatibility relations (see [21, Ch. II, §2]), namely (for all $X, X_{1}, X_{2} \in \operatorname{MTM}\left(\mathrm{Gr}_{G}\right)^{\mathrm{c}}$, $\left.\tau: X_{1} \rightarrow X_{2}\right)$

(i) $g_{1}=\mathrm{id}_{R}$,

(ii) $g_{X_{1} \star X_{2}}=g_{X_{1}} \otimes g_{X_{2}}$, and

(iii) $g_{X_{2}} \circ \tau=(\omega(\tau) \otimes 1) \circ g_{X_{1}}$.

The map $\widetilde{G}(R) \rightarrow \widehat{G}_{1}(R)$ sends such a family $g:=\left(g_{X}\right)_{X \in \mathrm{MTM}(\mathrm{Gr})}$ to the collection of automorphisms, where $X$ only lies in the $\star$-subcategory $\operatorname{Sat}_{G} \subset \operatorname{MTM}\left(\mathrm{Gr}_{G}\right)$. Likewise for $e_{*} \operatorname{MTM}(S) \subset \operatorname{MTM}\left(\mathrm{Gr}_{G}\right)$. The injectivity of $\alpha$ therefore follows from Corollary 5.6: any motive in $\operatorname{MTM}\left(\mathrm{Gr}_{G}\right)$ is (isomorphic to one) of the form

$$
X=\bigoplus_{i=1}^{n} \mathrm{IC}_{L_{i}, \mu_{i}}=\bigoplus_{i=1}^{n}(\underbrace{e_{*} L_{i}}_{\in e_{*} \operatorname{MTM}(S)} \star \underbrace{\mathrm{IC}_{\mu_{i}}}_{\in \operatorname{Sat}_{G}})
$$

Thus, $g_{X}=\bigoplus_{i} g_{e_{*} L_{i}} \otimes g_{\mathrm{IC}_{\mu_{i}}}$ is trivial if $\left.g\right|_{\mathrm{Sat}_{G}}=\mathrm{id}$ and $\left.g\right|_{e_{*} \operatorname{MTM}(S)}=\mathrm{id}$.

Given families of automorphisms $h:=\left(h_{X}\right)_{X \in \operatorname{Sat}_{G}}$ and $h^{\prime}:=\left(h_{Y}^{\prime}\right)_{Y \in e_{*} \operatorname{MTM}(S)}$ such that $h_{X}=h_{X}^{\prime}$ for $X \in i_{*} \operatorname{MTM}(S)^{\text {pure }}$, the surjectivity of $\alpha(R)$ requires to glue these families of automorphisms to one on $\operatorname{MTM}\left(\mathrm{Gr}_{G}\right)$. To show this we use that there is an isomorphism

$$
\mathrm{IC}_{\mu}(n) \star e_{*} L \simeq \mathrm{IC}_{\mu^{\prime}}\left(n^{\prime}\right) \star e_{*} L^{\prime}
$$

(if and) only if $\mu=\mu^{\prime}$ and $L^{\prime} \simeq L\left(n-n^{\prime}\right)$. Indeed the former follows from support considerations, the latter follows by restricting the isomorphism of motives to $\mathrm{Gr}_{G}^{\mu} \subset$ $\mathrm{Gr}_{G}^{\leq \mu}$. For a motive $X$ as above, we can therefore define $\lambda_{X}:=h_{\mathrm{IC}_{\mu}(n)} \otimes h_{e_{*} L}^{\prime}$ independently of the presentation of $X$ as a convolution product. We extend this additively. For a morphism $\tau: X:=\bigoplus_{i} \mathrm{IC}_{\mu_{i}, L_{i}} \rightarrow \bigoplus_{i^{\prime}} \mathrm{IC}_{\mu_{i^{\prime}}^{\prime}, L_{i^{\prime}}^{\prime}}=: X^{\prime}$, we obtain $g_{X^{\prime}} \circ \tau=(\omega(\tau) \otimes 1) \circ g_{X}$ by the description of the Hom group in Lemma 6.3 and the corresponding functoriality property for $h^{\prime}$.

\subsection{Extension to Ind-Categories}

The equivalences in Theorems 6.8 and 6.14 admit the following extensions to not necessarily compact objects. Such a statement can be useful in contexts when one wants to invoke adjoint functor theorems.

Corollary 6.15 There are equivalences of symmetric monoidal abelian $\mathbf{Q}$-linear categories

$$
\operatorname{Sat}_{G}=\operatorname{Rep}_{\mathbf{Q}}\left(\widehat{G}_{1}\right),
$$




$$
\operatorname{MTM}\left(L^{+} G_{S} \backslash L G_{S} / L^{+} G_{S}\right)=\operatorname{MTM}\left(\operatorname{Gr}_{G, S}\right)=\operatorname{Rep}_{\mathbf{Q}}\left(\mathscr{U}_{S} \rtimes \widehat{G}_{1}\right),
$$

where $\operatorname{Rep}_{\mathbf{Q}}$ denotes the category of not necessarily finite-dimensional representations.

Proof Any representation of a flat group scheme, in particular any representation of a pro-algebraic group such as $\mathscr{U}_{S} \rtimes \widehat{G}_{1}$ is locally finite [39, §I.2.13]. As finitedimensional representations are compact objects, the category $\operatorname{Rep}_{\mathbf{Q}}\left(\mathscr{U}_{S} \rtimes \widehat{G}_{1}\right)$ is compactly generated by its subcategory $\operatorname{Rep}_{\mathbf{Q}}\left(\mathscr{U}_{S} \rtimes \widehat{G}_{1}\right)^{\mathrm{fd}}$. On the other hand, $\operatorname{MTM}\left(\mathrm{Gr}_{G}\right)$ is compactly generated by $\operatorname{MTM}\left(\mathrm{Gr}_{G}\right)^{\mathrm{c}}$ by virtue of [60, Prop. 3.2.15]. Similarly, $\mathrm{Sat}_{G}$ is compactly generated by definition. We therefore obtain the claim by applying the ind-completion to the equivalence in Theorem 6.14.

\subsection{From motives to functions}

Let $S=\operatorname{Spec}\left(\mathbf{F}_{q}\right)$ be the spectrum of a finite field. We consider the (spherical) Hecke ring

$$
\mathcal{H}_{G} \stackrel{\text { def }}{=} \mathcal{C}_{c}\left(L^{+} G\left(\mathbf{F}_{q}\right) \backslash L G\left(\mathbf{F}_{q}\right) / L^{+} G\left(\mathbf{F}_{q}\right) ; \mathbf{Z}\right)
$$

where the ring structure is given by the convolution of functions, cf. [33, §2]. This ring has a $\mathbf{Z}$-basis given by the characteristic functions $c_{\mu}, \mu \in X_{*}(T)^{+}$on the double cosets $L^{+} G\left(\mathbf{F}_{q}\right) \varpi^{\mu} L^{+} G\left(\mathbf{F}_{q}\right)$. Taking the trace of geometric Frobenius on motives as in [16] induces a surjective ring morphism

$$
K_{0} \mathrm{Sat}_{G}^{\mathrm{c}} \rightarrow \mathcal{H}_{G} \otimes_{\mathbf{Z}} \mathbf{Z}\left[q^{-1}\right], \quad M \mapsto f_{M},
$$

where $K_{0} \mathrm{Sat}_{G}^{c}$ denotes the Grothendieck ring.

Recall that the trace of the geometric Frobenius on $\mathbf{Q}_{\ell}(1)$ is $q^{-1}$, cf. [19, (1.2.5) (iv)]. Thus, for the trivial group $G=1$, the preceding map is the ring homomorphism $\mathbf{Z}\left[t, t^{-1}\right] \rightarrow \mathbf{Z}\left[q^{-1}\right]$ sending the class $t$ of $\mathbf{Q}(1)$ to $q^{-1}$.

In general, if $M$ is any stratified Tate motive on $\mathrm{Gr}_{G}$ (with respect to the stratification into $L^{+} G$-orbits), then its restriction $\iota_{\mu}^{*} M$ to some Schubert cell $\mathrm{Gr}_{G}^{\mu}$ lies in DTM $\left(\mathrm{Gr}_{G}^{\mu}\right)$ by construction. Thus, if $M$ is also compact, then $\iota_{\mu}^{*} M$ is a finite successive extension of Tate motives $1_{\mu}(n)[m], n, m \in \mathbf{Z}$. This immediately implies that the function $f_{M}$ takes values in $\mathbf{Z}\left[q^{-1}\right]$ as opposed to $\mathbf{Q}$. Also since $\iota_{\mu}^{*} \mathrm{IC}_{\mu}$ coincides with $1_{\mu}$ up to a shift, the function $c_{\mu}$ appears with multiplicity \pm 1 in $f_{\mathrm{IC}_{\mu}}$ which shows that (6.16) is surjective.

Let $R\left(\widehat{G}_{1}\right)$ be the Grothendieck ring of the category $\operatorname{Rep}_{\mathbf{Q}}^{\mathrm{fd}}\left(\widehat{G}_{1}\right)$. By construction there is a morphism $d: \widehat{G}_{1} \rightarrow \mathbf{G}_{m, \mathbf{Q}},[(g, \lambda)] \mapsto \lambda^{2}$. When viewed as a representation this is nothing but the highest weight representation $V_{0}(1)$ of $\widehat{G}_{1}$. We denote its class in $R\left(\widehat{G}_{1}\right)$ by $\left[V_{0}(1)\right]=[d]$.

Corollary 6.17 The motivic Satake equivalence in Theorem 6.8 induces an isomorphism of rings

$$
\mathcal{H}_{G} \otimes \mathbf{Z} \mathbf{Z}\left[q^{-1}\right] \stackrel{\simeq}{\longrightarrow} R\left(\widehat{G}_{1}\right) /\left(\left[d^{-1}\right]-q\right) .
$$


Proof The motivic Satake equivalence induces an isomorphism on Grothendieck rings

$$
K_{0} \operatorname{Sat}_{G}^{c} \simeq K_{0} \operatorname{Rep}_{\mathbf{Q}}^{\mathrm{fd}}\left(\widehat{G}_{1}\right)=R\left(\widehat{G}_{1}\right)
$$

under which the class $\left[\mathrm{IC}_{0}(-1)\right]$ corresponds to $\left[V_{0}(-1)\right]=\left[d^{-1}\right]$. Hence, it is enough to show that the kernel of (6.16) is the principal ideal generated by $a:=$ $\left[\mathrm{IC}_{0}(-1)\right]-q\left[\mathrm{IC}_{0}\right]$. As the geometric Frobenius acts on $\mathbf{Q}(-1)$ by multiplication with $q$, the class $a$ lies in the kernel. Conversely, by induction one easily sees that all classes $\left[\mathrm{IC}_{0}(-n)\right]-q^{n}\left[\mathrm{IC}_{0}\right], n \in \mathbf{Z}$ lie in the principal ideal generated by $a$. An elementary calculation using that the classes $\left[\mathrm{IC}_{\mu}\right], \mu \in X_{*}(T)^{+}$are linearly independent implies the corollary.

Remark 6.18 We note that the isomorphism of $\mathbf{Q}$-groups $\widehat{G} \times \mathbf{G}_{m, \mathbf{Q}} \rightarrow \widehat{G} \times \mathbf{G}_{m, \mathbf{Q}}$, $(g, \lambda) \mapsto\left(g, \lambda^{-1}\right)$ induces an isomorphism $\widehat{G}_{1} \simeq \widehat{G}_{1}$, and hence an isomorphism of rings

$$
R\left(\widehat{G}_{1}\right) /\left(\left[d^{-1}\right]-q\right) \simeq R\left(\widehat{G}_{1}\right) /([d]-q) .
$$

Under the Tannakian dictionary this normalization corresponds to a sign change in the weight graduation in Definition 5.11. For a concrete example take $G=\mathrm{PGL}_{2}$ so that $\widehat{G}=\mathrm{SL}_{2}$. Since $(2 \rho)(-1)$ is the diagonal matrix $\operatorname{diag}(-1,-1)$, we get $\widehat{G}_{1}=\mathrm{GL}_{2}$ and $d=\operatorname{det}: \mathrm{GL}_{2} \rightarrow \mathbf{G}_{m}$. Thus, the Satake isomorphism with the new normalisation reads in this case

$$
\mathcal{H}_{\mathrm{PGL}_{2}} \otimes \mathbf{Z} \mathbf{Z}\left[q^{-1}\right] \stackrel{\simeq}{\longrightarrow} R\left(\mathrm{GL}_{2}\right) /([\operatorname{det}]-q)
$$

If $\mu \in X_{*}(T)^{+}=\mathbf{Z}_{\geq 0}$, then this isomorphism explicitly is given by

$$
f_{\mathrm{IC}_{\mu}} \mapsto\left[\operatorname{Sym}^{\mu} \mathbf{Q}^{2}\right]
$$

where $\left[\operatorname{Sym}^{\mu} \mathbf{Q}^{2}\right]$ denotes the class of the $\mu$-th symmetric power of the standard representation of $\mathrm{GL}_{2}$.

Acknowledgements We thank Dennis Gaitsgory, Fritz Hörmann, and Thomas Nikolaus for helpful discussions, and the anonymous referee for many suggestions that improved the quality of the manuscript. The authors thank the University of Münster, Harvard University, Deutsche Forschungsgemeinschaft (DFG, German Research Foundation under Germany's Excellence Strategy EXC 2044-390685587, Mathematics Münster: Dynamics-Geometry-Structure), the Institut de Mathématiques de Jussieu and the Technische Universität Darmstadt for financial and logistical support which made this research possible.

Funding Open Access funding enabled and organized by Projekt DEAL

Open Access This article is licensed under a Creative Commons Attribution 4.0 International License, which permits use, sharing, adaptation, distribution and reproduction in any medium or format, as long as you give appropriate credit to the original author(s) and the source, provide a link to the Creative Commons licence, and indicate if changes were made. The images or other third party material in this article are included in the article's Creative Commons licence, unless indicated otherwise in a credit line to the material. If material is not included in the article's Creative Commons licence and your intended use is not permitted by statutory regulation or exceeds the permitted use, you will need to obtain permission directly from the copyright holder. To view a copy of this licence, visit http://creativecommons.org/licenses/by/4.0/. 


\section{Appendix A: Complements on motives}

In this appendix, we show how the well-known compatibilities (stated in this form in [40])

$$
\begin{aligned}
& \left(f_{1} \times f_{2}\right)^{*}\left(M_{1} \otimes M_{2}\right) \cong\left(f_{1}^{*} M_{1}\right) \otimes\left(f_{2}^{*} M_{2}\right) \\
& \left(f_{1} \times f_{2}\right) !\left(M_{1} \otimes M_{2}\right) \cong\left(f_{1 !} M_{1}\right) \otimes\left(f_{2 !} M_{2}\right)
\end{aligned}
$$

of the exterior product $\otimes$ of motives with $f^{*}$ and $f$ ! can be coherently organized. This will be used to establish the structure of a symmetric lax monoidal functor for the presheaf $\mathrm{DM}^{!}$when we restrict to placid morphisms between placid schemes, such as the $L^{+} G$-torsors $L G^{\leq \mu} \rightarrow \mathrm{Gr}^{\leq \mu}$. This is used in the discussion of the convolution product in Sect. 3.

Throughout $\S$ A, we assume the base scheme $S$ to be a Noetherian separated scheme of finite Krull dimension. We note that all separatedness assumptions (here, on $S$, and below on certain maps of $S$-schemes) could eventually be dropped by proceeding as in [60, Prop. 2.1.14].

\section{A.1. The exterior product}

We equip the categories $\operatorname{Sch}_{S}^{\mathrm{ft}}, \mathrm{Sch}_{S}$ and Cat, Cat $\infty$, the $(\infty$-)category of all small $(\infty$-)categories, with their cartesian symmetric monoidal structure [49, §2.4.1].

In particular, we consider the cocartesian fibration $\left(\mathrm{Sch}_{S}^{\mathrm{ft}}\right)^{\times} \rightarrow$ Fin taking values in the category of finite pointed sets. Recall that the objects of $\left(\operatorname{Sch}_{S}^{\mathrm{ft}}\right)^{\times}$are sequences $\left(X_{1}, \ldots, X_{n}\right)$ with $X_{i} \in \operatorname{Sch}_{S}^{\mathrm{ft}}$ and, among others, the category has morphisms of the form $\left(X_{1}, \ldots, X_{n}\right) \rightarrow X_{1} \times{ }_{S} \cdots \times{ }_{S} X_{n}$, corresponding to $\operatorname{id}_{X_{1} \times \cdots \times X_{n}}$. Let $\left(\mathrm{Sch}_{S}^{\mathrm{ft}}\right)^{\times, \vee} \rightarrow$ Fin ${ }^{\text {op }}$ be the associated cartesian fibration as constructed in [6]. The opposite of this, which is again a cocartesian fibration, encodes the usual symmetric monoidal structure on $\left(\mathrm{Sch}_{S}^{\mathrm{ft}}\right)^{\mathrm{op}}$. We will abbreviate the source of this map as $\left(\mathrm{Sch}_{S}^{\mathrm{ft}}\right)^{\mathrm{op}, \times}$ or even just $\left(\operatorname{Sch}_{S}^{\mathrm{ft}}\right)^{\mathrm{op}}$.

The subcategory $\operatorname{AffSch}_{S}^{\mathrm{ft}} \subset \operatorname{Sch}_{S}^{\mathrm{ft}}$ (consisting of affine finite type $S$-schemes, Spec $R \rightarrow S$ ) is closed under the product since $S$ is by assumption separated. We further endow DGCat ${ }_{\text {cont }}$ and $\operatorname{Pr}^{\mathrm{L}}$ (presentable $\infty$-categories with colimit-preserving functors) with the Lurie tensor product, see e.g. [29, Ch. 1, §6].

Lemma A.2 The functor

$$
\mathrm{DM}^{*}:\left(\mathrm{Sch}_{S}^{\mathrm{ft}}\right)^{\mathrm{op}} \rightarrow \mathrm{DGCat}_{\mathrm{cont}}
$$

admits a natural symmetric lax monoidal structure such that for finite type $S$-schemes $X_{1}, X_{2}$, this structure is the exterior product

$$
\bigotimes: \operatorname{DM}\left(X_{1}\right) \otimes \operatorname{DM}\left(X_{2}\right) \rightarrow \operatorname{DM}\left(X_{1} \times X_{2}\right)
$$


Proof The functor $\mathrm{Sch}_{S}^{\mathrm{ft}, \text { op }} \rightarrow$ Cat, $X \mapsto \mathrm{Sm} / X$ is symmetric lax monoidal (with respect to the cartesian monoidal structures on both categories) by means of the exterior product. The inclusion Cat $\rightarrow \mathrm{Cat}_{\infty}$ is symmetric monoidal. The presheaf functor (in the $\infty$-categorical sense) $\mathcal{P}: \mathrm{Cat}_{\infty} \rightarrow \operatorname{Pr}^{\mathrm{L}}$ is symmetric monoidal [49, Rem. 4.8.1.8]. Thus, the composite $X \mapsto \mathcal{P}(\mathrm{Sm} / X)$ is symmetric lax monoidal. In addition, the (nonfull) subcategory $W_{X} \subset \mathcal{P}(\mathrm{Sm} / X)$ consisting of the usual $\mathbf{A}^{1}$-projections and étale hypercoverings are monoidal subcategories, so that the functor $X \mapsto\left(\mathcal{P}(\mathrm{Sm} / X), W_{X}\right)$ is a symmetric monoidal functor taking values in the $\infty$-category $\mathrm{WCat}_{\infty}$ of relative $\infty$-categories. The localization functor $\mathrm{WCat}_{\infty} \rightarrow \mathrm{Cat}_{\infty},(C, W) \mapsto C\left[W^{-1}\right]$ is symmetric lax monoidal [49, Prop. 4.1.7.2, Prop. 4.1.7.4].

The stabilization process, i.e., turning $\mathbf{P}^{1}$ into an invertible object, is also a symmetric lax monoidal functor. This is readily apparent from the description of this process in $[62, \S 4.1]$ : abbreviating the notation of loc. cit. as $P:=\mathcal{P}\left(\text { free }^{\otimes}(\Delta[0])\right)^{\otimes}$ and

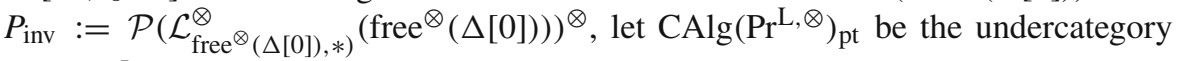
$\mathrm{CAlg}\left(\operatorname{Pr}^{\mathrm{L}, \otimes}\right)_{P /}$. Its objects are pairs $(C, X)$ consisting of a presentable symmetric monoidal $\infty$-category $C$ and an object $X \in C$. Similarly, consider the undercategory $\mathrm{CAlg}\left(\operatorname{Pr}^{\mathrm{L}, \otimes}\right)_{\mathrm{pt}, \text { inv }}:=\mathrm{CAlg}\left(\operatorname{Pr}^{\mathrm{L}, \otimes}\right)_{P_{\text {inv }} /}$ whose objects consist of similar pairs $(C, X)$, but where $X$ is a $\otimes$-invertible object. The objects $P$ and $P_{\text {inv }}$ have natural comonoid structures stemming from the comonoid structure present on any object in a cartesian symmetric monoidal category such as $\mathrm{Cat}_{\infty}^{\times}$. Thus, the undercategories under these two objects have a natural symmetric monoidal structure in such a way that the functor $(C, X) \mapsto X$ is symmetric monoidal. The natural functor, arising from the map $P \rightarrow P_{\text {inv }}$,

$$
\mathrm{CA} \lg \left(\operatorname{Pr}^{\mathrm{L}, \otimes}\right)_{P_{\text {inv }} /} \rightarrow \mathrm{CA} \operatorname{Cg}\left(\operatorname{Pr}^{\mathrm{L}, \otimes}\right)_{P /}
$$

is symmetric monoidal. Hence its left adjoint, which by [62, Def. 4.1.8] is the functor mapping a pointed category $(C, X)$ to $\left(C\left[X^{-1}\right], X\right)$, is symmetric lax monoidal. This abstract observation is applied to the functor $\operatorname{Sch}_{S}^{\mathrm{ft}, \text { op }} \rightarrow \mathrm{CAlg}\left(\operatorname{Pr}^{\mathrm{L}, \otimes}\right)_{P /}$, $X \mapsto\left(\mathcal{P}(\mathrm{Sm} / X)\left[\left\langle\mathbf{A}^{1} \text {, et }\right\rangle^{-1}\right], \mathbf{P}_{X}^{1}\right)$ which is symmetric lax monoidal by the above $\left(\right.$ and $\left.\mathbf{P}_{X}^{1} \times \mathbf{P}_{Y}^{1}=\mathbf{P}_{X \times Y}^{1}\right)$.

The composite, denoted by $\mathrm{SH}^{*}: \operatorname{Sch}_{S}^{\mathrm{ft}, \mathrm{op}} \rightarrow \operatorname{Pr}^{\mathrm{L}, \otimes}$ takes values in $\operatorname{Pr}_{\text {stb }}^{\mathrm{L}, \otimes}$, the $\infty$-category of stable presentable symmetric monoidal $\infty$-categories and colimitpreserving functors and is by the above a symmetric lax monoidal functor. Finally, DM arises from composing with the symmetric monoidal functor $\operatorname{Pr}_{\text {stb }}^{\mathrm{L}}=\operatorname{Mod}_{\mathrm{Sp}}\left(\operatorname{Pr}^{\mathrm{L}}\right) \stackrel{-\otimes \mathbf{Q}}{\longrightarrow}$ $\operatorname{Mod}_{\mathbf{Q}}\left(\operatorname{Pr}_{\text {stb }}^{\mathrm{L}}\right)=$ : DGCat cont $_{\text {. }}$

We now retrace the construction of $\mathrm{DM}_{1}^{*}$ as a functor out of the category of correspondences, by keeping track of the symmetric lax monoidal structure and thus of projection formulas, including all their higher coherences. We use, in the same vein as Hoyois [38] and Khan [43], the universal property of the category of correspondences. An alternative approach for coherently encoding projection formulas avoiding the category of correspondences appears in $[1, \S 4.5]$. 
Recall the $(\infty, 2)$-category of correspondences $\operatorname{Corr}\left(\left(\operatorname{Sch}_{S}^{\mathrm{ft}}\right)^{\times, \vee}\right)_{\text {horiz,vert }}^{\text {adm }}$ defined in [29, §7]. Here horiz, vert and adm are certain subcategories of $\left(\mathrm{Sch}_{S}^{\mathrm{ft}}\right)^{\times, \vee}$, to be specified below more concretely. The objects of this category are the objects $X \in$ $\left(\mathrm{Sch}_{S}^{\mathrm{ft}}\right)^{\times, \vee}$ (which are, in their turn, finite collections of objects in $\mathrm{Sch}_{S}^{\mathrm{ft}}$ ); 1-morphisms from $X$ to $Y$ are spans of the form $Y \stackrel{g}{\leftarrow} Z \stackrel{f}{\rightarrow} X$ with $g \in$ vert and $f \in$ horiz, and 2-morphisms between such a morphism and another similar correspondence is a map $Z \rightarrow Z^{\prime}$ in adm that is compatible with the maps to $X$ and $Y$. This describes the lowdimensional data of this category, we refer to loc. cit. for the full definition including the $(\infty, 2)$-categorical structure.

Definition A.3 A map $f$ in a symmetric monoidal $\infty$-category $\mathcal{C}^{\otimes} \rightarrow$ Fin is called dormant if its image in Fin is an identity map.

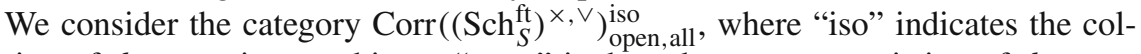
lection of dormant isomorphisms, "open" is the subcategory consisting of dormant morphisms

$$
\left(f_{1}, \ldots, f_{n}\right):\left(X_{1}, \ldots, X_{n}\right) \rightarrow\left(Y_{1}, \ldots, Y_{n}\right)
$$

where each $f_{i}$ is an open embedding. Finally, "all" means that no restriction is imposed on the horizontal morphisms.

The extension of the functor $\left(\mathrm{Sch}_{S}^{\mathrm{ft}}\right)^{\times, \vee} \rightarrow$ Fin $^{\text {op }}$ to correspondences, and restricting to the above subcategory gives a functor $\operatorname{Corr}\left(\left(\operatorname{Sch}_{S}^{\mathrm{ft}}\right)^{\times, \vee}\right)_{\text {open, all }}^{\text {iso }} \rightarrow$ Corr $\left(\text { Fin }^{\text {op }}\right)_{\text {id,all }}^{\text {iso }}=$ Fin. Given a map $\alpha:\langle m\rangle \rightarrow\langle n\rangle$ in Fin and a cocartesian lift $\left(X_{1}, \ldots, X_{n}\right) \rightarrow\left(Y_{1}, \ldots, Y_{m}\right)$ in $\left(\operatorname{Sch}_{S}^{\mathrm{ft}}\right)^{\mathrm{op}}$, on checks that $\left(X_{i}\right) \stackrel{\text { id }}{\leftarrow}\left(X_{i}\right) \rightarrow\left(Y_{j}\right)$ is a cocartesian lift in $\operatorname{Corr}\left(\left(\operatorname{Sch}_{S}^{\mathrm{ft}}\right)^{\times, \vee}\right)$. The Segal condition holds trivially by definition, so that $\operatorname{Corr}\left(\left(\mathrm{Sch}_{S}^{\mathrm{ft}}\right)^{\times, \vee}\right)_{\text {open, all }}^{\text {iso }}$ is a symmetric monoidal $\infty$-category.

Lemma A.4 The functor $\mathrm{DM}^{*}$ extends uniquely to a symmetric lax monoidal functor

$$
\mathrm{DM}_{\sharp}^{*}: \operatorname{Corr}\left(\left(\operatorname{Sch}_{S}^{\mathrm{ft}}\right)^{\times, \vee}\right)_{\text {open, all }}^{\text {iso }} \rightarrow \mathrm{DGCat}_{\text {cont }} .
$$

Proof We show the existence of the functor using [29, Chapter 7, Theorem 3.2.2(b)]. Among the general conditions in Chapter 7, 1.1.1 there, the only requirement to check is the existence of pullbacks in $\left(\mathrm{Sch}_{S}^{\mathrm{ft}}\right)^{\times, \vee}$ along maps in the subcategory "open". This is clear: given open embeddings $f_{i}$ and a map as in the horizontal arrow, i.e., a map $\alpha:\langle n\rangle \rightarrow\langle m\rangle$, and $g_{i}: Z_{i} \rightarrow \prod_{j \in \alpha^{-1}(i)} Y_{j}$, the diagram

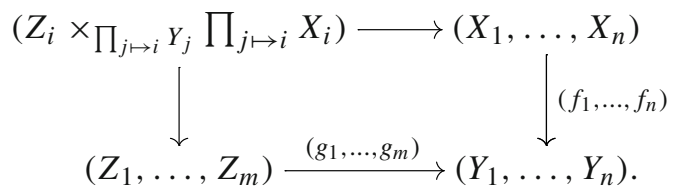

is cartesian in $\left(\mathrm{Sch}_{S}^{\mathrm{ft}}\right)^{\times, \vee}$ and the left vertical map is again in "open". In order to check the Beck-Chevalley condition, it suffices to separately consider the case where $\left(g_{i}\right)$ is 
inert, respectively active, since these form a factorization system. For inert morphisms, this is clear. For active morphisms, we may assume that $m=1$ and $n=2$ above, in which case we consider $g: Z_{1} \rightarrow Y_{1} \times Y_{2}$. Then the Beck-Chevalley condition is the assertion that the following diagram commutes, which follows from the construction of $\otimes$ :

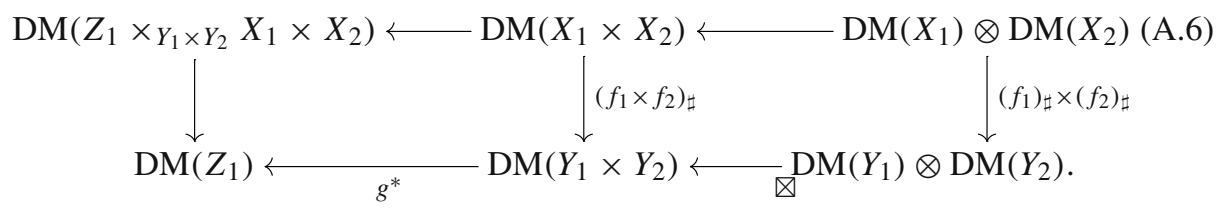

The functor $\mathrm{DM}_{\sharp}^{*}$ obtained in this way clearly preserves edges that are cocartesian over Fin, thus giving a symmetric lax monoidal functor.

Lemma A.7 Let sep, resp. proper be the subcategory of $\left(\mathrm{Sch}_{S}^{\mathrm{ft}}\right)^{\times}$spanned by morphisms that are dormant (i.e., map to an identity in Fin), and are componentwise separated (resp. proper). The functor $\mathrm{DM}_{\sharp}^{*}$ in (A.5) extends uniquely to a symmetric lax monoidal functor:

$$
\mathrm{DM}_{!}^{*}: \operatorname{Corr}\left(\left(\mathrm{Sch}_{S}^{\mathrm{ft}}\right)^{\times, \vee}\right)_{\text {sep,all }}^{\text {proper }} \rightarrow \mathrm{DGCat}_{\mathrm{cont}} .
$$

Proof We apply [29, Chapter 7, Thm. 5.2.7]. To check its assumptions note that a map in proper $\cap$ open is dormant, and componentwise an open embedding. Such a map is a monomorphism in $\left(\operatorname{Sch}_{S}^{\mathrm{ft}}\right)^{\times, \vee}$. The condition in Chapter 7, 5.2.2 there and also the Beck-Chevalley condition is satisfied since, again, the corresponding properties only need to be checked for maps in open, resp. proper, which are by definition dormant. Thus the Beck-Chevalley condition reduces to an assertion similar to the commutativity of (A.6), except that $(-)_{\sharp}$ is replaced by $(-)_{!}$, in other words, the classical projection formula as recalled in (A.1).

Corollary A.8 Write $\mathrm{Sm}_{S, \mathrm{sm} \cap \mathrm{ft} p}^{\mathrm{ft}}$ for the category consisting of smooth separated finitetype $S$-schemes and smooth separated morphisms. There is a natural isomorphism of functors

$$
\mathrm{Tw}:\left.\left.\mathrm{DM}^{*}\right|_{\mathrm{Sm}_{S, \text { smกsep }}^{\mathrm{ft}}} \Rightarrow \mathrm{DM}^{!}\right|_{\mathrm{Sm}_{S, \mathrm{sm} \cap \mathrm{sep}}^{\mathrm{ft}}}:\left(\mathrm{Sm}_{S, \text { smกsep }}^{\mathrm{ft}}\right)^{\mathrm{op}} \rightarrow \mathrm{DGCat}_{\mathrm{cont}},
$$

whose evaluation at a map $f: X \rightarrow Y$ is the natural transformation

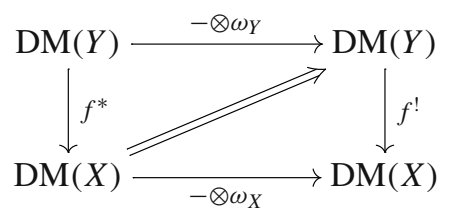

stemming from the projection formula. Here, $\omega_{X}:=p_{X}^{!} 1$ with $p_{X}: X \rightarrow S$ the structural map. 
Proof Any map $X \stackrel{f}{\rightarrow} Y$ of schemes naturally gives rise to a pair $(Y, X)$, where $Y$ is a comonoid and $X$ is a $Y$-comodule (both with respect to the cartesian monoidal structure) on Sch. The coaction is given by $X \stackrel{\Delta_{X}}{\rightarrow} X \times X \stackrel{f \times \text { id }}{\rightarrow} Y \times X$. Applying the symmetric lax monoidal functor $\mathrm{DM}_{!}^{*}$ to this object, we obtain an object in $\mathcal{L} \mathcal{M}$ (DGCat Dont $_{\text {c }}$, namely the commutative algebra object $\operatorname{DM}(Y)$, and the $\operatorname{DM}(Y)$ module $\operatorname{DM}(X)$, where the action arises as

$$
\mathrm{DM}(Y) \otimes \mathrm{DM}(X) \stackrel{\otimes}{\rightarrow} \mathrm{DM}(Y \times X) \stackrel{(f \times \mathrm{id})^{*}}{\rightarrow} \mathrm{DM}(X \times X) \stackrel{\Delta_{X}^{*}}{\rightarrow} \mathrm{DM}(X)
$$

This can also be computed as the natural action of $\mathrm{DM}(Y)$, via $f^{*}$, on the symmetric monoidal category $\operatorname{DM}(X)$ (equipped with its usual $\otimes$ ).

The map $f$ also gives rise to a map of comodule objects $(Y, X) \rightarrow(Y, Y)$. Applying the functor $\mathrm{DM}_{!}^{*}$ to the map of induced left module objects, namely the pair

$$
\left(\text { id, } f_{!}\right):(\operatorname{DM}(Y), \operatorname{DM}(X)) \rightarrow(\operatorname{DM}(Y), \operatorname{DM}(Y)) .
$$

(Such an interpretation of the projection formula was observed by Khan [43]. Note, however, that the approach to projection formulas laid out in op. cit. does not seem to work as is, since the morphisms in Ch. 2, \$4.1.6 there cannot be composed.) This map admits a left adjoint separately for each object in $\mathcal{L} \mathcal{M}$, namely id and $f^{!}$, respectively. By [49, Cor. 7.3.2.7], the functor therefore admits a right adjoint relative to $\mathcal{L} \mathcal{M}^{\otimes}$, still denoted $f$ !

This in particular expresses the existence of a natural map $f^{*} A \otimes f^{!} B \rightarrow f^{!}(A \otimes B)$ that is functorial in $A$ and $B$. It follows from the naturality of the construction that it is also functorial in $f$. The sought-for transformation is defined as the restriction of this map to $B=\omega_{Y}$. By relative purity, this map is an isomorphism whenever $f$ is smooth. Moreover, for $X$ and $Y$ smooth, $\omega_{X}$ and $\omega_{Y}$ are $\otimes$-invertible.

Lemma A.9 Consider the functor

$$
\mathrm{DM}^{!}:\left(\operatorname{Sch}_{S, \text { sep }}^{\mathrm{ft}}\right)^{\times, \vee} \rightarrow \mathrm{DGCat}_{\mathrm{cont}}
$$

obtained from the composite $\left(\operatorname{Sch}_{S, \mathrm{sep}}^{\mathrm{ft}}\right)^{\times, \vee} \rightarrow \operatorname{Corr}\left(\left(\operatorname{Sch}_{S}^{\mathrm{ft}}\right)^{\times, \vee}\right)_{\text {sep, all }}^{\text {proper }} \stackrel{\mathrm{DM}_{!}^{*}}{\rightarrow} \mathrm{DGCat}_{\text {cont }}$ by passing to right adjoints.

- This functor is symmetric lax monoidal if $S=\operatorname{Spec} k$ is a field.

- The restriction of this functor to $\left(\mathrm{Sch}_{S, \mathrm{sm} \cap \mathrm{sep}}^{\mathrm{ft}}\right)^{\times, \vee}$ is symmetric lax monoidal for general $S$. Here the subscript $\mathrm{sm} \cap \mathrm{sep}$ refers to the (non-full, symmetric monoidal) subcategory comprising all finite type $S$-schemes, but only smooth separated maps.

The fiber over $\langle 1\rangle$ of $\left(\mathrm{Sch}_{S, \mathrm{sm} \cap \mathrm{sep}}^{\mathrm{ft}}\right)^{\times, \vee}$ identifies with $\left(\mathrm{Sch}_{S, \mathrm{sm} \cap \mathrm{sep}}^{\mathrm{ft}}\right)^{\mathrm{op}}$, and we also denote this symmetric lax monoidal functor by $\mathrm{DM}^{!}:\left(\mathrm{Sch}_{S, \mathrm{sm} \cap \mathrm{sep}}^{\mathrm{ft}}\right)^{\mathrm{op}} \rightarrow \mathrm{DGCat}_{\mathrm{cont}}$.

Proof The functor $\mathrm{DM}^{!}$exists as stated, since the right adjoints happen to preserve colimits as well. (This is well-known and uses the assumptions that $S$ is Noetherian and 
of finite Krull dimension.) To check it is a symmetric lax monoidal functor it remains to check that for two maps $f_{1}, f_{2}$ in $\operatorname{Sch}_{S}^{\mathrm{ft}}$ the natural map $\left(f_{1}\right)^{!} M_{1} \otimes\left(f_{2}\right)^{!} M_{2} \rightarrow$ $\left(f_{1} \times f_{2}\right)^{!}\left(M_{1} \otimes M_{2}\right)$ is an isomorphism. If $S$ is a field, this holds by [40, Prop. 2.3.5] (note this is nontrivial and uses alterations). For general $S$, but smooth maps $f_{i}$, this holds by relative purity and (A.1) for $*$-pullbacks.

\section{A.2. Motives on placid prestacks}

For a regular cardinal $\kappa$, recall from Definition 2.4 the category $\operatorname{AffSch}_{S}^{\kappa}$. It consists of those affine schemes that can be presented as $\kappa$-small cofiltered limits

$$
X=\lim X_{i},
$$

where the $X_{i}$ are affine finite type $S$-schemes.

Definition A.11 ([28, App. C], [56, Def. 4.2.1]) An object in $\mathrm{AffSch}_{S}^{\kappa}$ is called placid if it admits a placid presentation, i.e., one such that the transition maps $X_{i} \rightarrow X_{j}$ in (A.10) are smooth (and necessarily affine). A map between two such placid affine $S$-schemes is called placid if for any pair of placid presentations $X=\lim X_{i}, Y=$ $\lim Y_{j}$, and any $j$, there is some $i$ such that $X \rightarrow Y \rightarrow Y_{j}$ factors as $X \rightarrow X_{i} \rightarrow$ $Y_{j}$, where the second map is smooth. (It follows from [56, Lemma 4.5.1] that this condition only needs to be checked for any fixed presentations of $X$ and $Y$.) The non-full subcategory of $\operatorname{AffSch}_{S}^{\kappa}$ consisting of placid affine schemes and placid maps is denoted by $\operatorname{AffSch}_{S}^{\kappa, \mathrm{pl}}$. (Equivalently, [56, Rem. 4.10.3], $\operatorname{AffSch}_{S}^{\kappa, \mathrm{pl}}$ can be defined as the pro-category $\operatorname{Pro}_{\kappa \text {-small }}\left(\mathrm{AffSch}_{S, \mathrm{sm}}^{\mathrm{ft}}\right)$, the pro-completion of affine schemes of finite type over $S$ with smooth maps.)

We call the category $\operatorname{PreStk}_{S}^{\mathrm{pl}}:=\operatorname{Fun}\left(\operatorname{AffSch}_{S}^{\kappa, \mathrm{pl}}, \infty-\mathrm{Gpd}\right)$ the category of placid prestacks. It is the free completion of $\operatorname{AffSch}_{S}^{\kappa, \mathrm{pl}}$ under arbitrary colimits.

The restriction of prestacks to $\operatorname{AffSch}_{S}^{\kappa, \mathrm{pl}}$ induces an adjunction $\operatorname{PreStk}_{S}^{\mathrm{pl}} \leftrightarrows$ PreStk $_{S}$. A prestack is called placid if it lies in the essential image of the functor $\operatorname{PreStk}_{S}^{\mathrm{pl}} \rightarrow \operatorname{PreStk}_{S}$.

Example A.12 The groups $\mathcal{P}_{\mathbf{f}}$ (in particular the positive loop group $L^{+} G$ ) are placid affine $S$-schemes, provided that $S$ itself is affine. So the preimage of any open affine subscheme under the $\mathcal{P}_{\mathbf{f}}$-torsor $L G^{\leq w} \rightarrow \mathrm{Fl}^{\leq w}$ (see Sect. 3.2) is placid affine.

Thus, for any pair of facets $\mathbf{f}, \mathbf{f}^{\prime}$, the double quotient of the $\mathcal{P}_{\mathbf{f}^{\prime}} \times \mathcal{P}_{\mathbf{f}}$-action (from the right and the left) on $L G^{\leq w}$ exists as a placid prestack $\mathcal{P}_{\mathbf{f}^{\prime}} \backslash L G^{\leq w} / \mathcal{P}_{\mathbf{f}}$. More generally, for any quasi-compact closed subscheme $X \subset L G$ the prestack $\mathcal{P}_{\mathbf{f}^{\prime}} \backslash X / \mathcal{P}_{\mathbf{f}}$ is placid.

Our goal is to have $\triangle$-products for motives on placid prestacks. By means of the following two results, this is a formal consequence of the symmetric lax monoidality of $\mathrm{DM}^{!}$on $\mathrm{AffSch}_{S, \mathrm{sm}}^{\mathrm{ft}} \subset \mathrm{Sch}_{S, \text { smกsep}}^{\mathrm{ft}}$.

Lemma A.13 Let $\mathcal{C}$ be a small symmetric monoidal $\infty$-category and $\mathcal{D}$ be a cocomplete symmetric monoidal $\infty$-category whose tensor product preserves colimits 
separately in each variable. Fix a regular cardinal $\kappa$. Then the restriction functor $\operatorname{Fun}\left(\operatorname{Ind}_{\kappa \text {-small }}(\mathcal{C}), \mathcal{D}\right)^{\otimes} \rightarrow \operatorname{Fun}(\mathcal{C}, \mathcal{D})^{\otimes}$ admits a symmetric monoidal left adjoint, where the monoidal structure on the functor categories is given by Day convolution.

Thus, any symmetric lax monoidal functor $F: \mathcal{C} \rightarrow \mathcal{D}$ can be left Kan extended to a symmetric lax monoidal functor on the ind-completion $\operatorname{Ind}_{\kappa \text {-small }}(\mathcal{C})$.

Proof This can be proven as in [53, Cor. 3.8]. The assumption in loc. cit. that $\mathcal{D}$ is accessible is, for the particular situation considered here, not needed since the invokation of the adjoint functor theorem in the proof of loc. cit. can be replaced by the universal property of the ind-completion [48, Prop. 5.3.5.10]. The last statement follows from the equivalence of symmetric lax monoidal functors and commutative monoid objects in the functor category under the Day convolution [31, Prop. 2.12].

In the next statement, $\operatorname{PreStk}_{S}^{(\mathrm{pl})}$ is equipped with the cartesian symmetric monoidal structure.

Corollary A.14 The functor $\mathrm{DM}^{*}$ on $\left(\mathrm{AffSch}_{S}^{\mathrm{ft}}\right)^{\mathrm{op}}$ admits a natural symmetric lax monoidal extension to $\mathrm{AffSch}_{S}^{\kappa}$ and to $\operatorname{PreStk}_{S}$. The same is true for $\mathrm{DM}^{!}$provided that $S=$ Spec $k$ is a field. Finally, the functor DM! admits a symmetric lax monoidal extension to $\mathrm{AffSch}_{S}^{\kappa, \mathrm{pl}}$ and to $\operatorname{PreStk}_{S}^{\mathrm{pl}}$.

Proof First, apply Lemma A.13 to $\mathcal{C}=\left(\mathrm{AffSch}_{S}^{\mathrm{ft}}\right)^{\mathrm{op}}$. Second, in order to extend DM* from $\mathrm{AffSch}_{S}$ to a symmetric lax monoidal functor on $\operatorname{PreStk}_{S}^{\times}$we use the argument in [29, Chapter 9, Prop. 3.2.4], according to which it is enough to observe that for any prestacks $F_{1}, \ldots, F_{n}$, the map

$$
\lim _{X \in \operatorname{AffSch}_{S}, X \rightarrow \prod F_{i}} \operatorname{DM}(X) \rightarrow \lim _{X_{i} \in \operatorname{AffSch}_{S}, X_{i} \rightarrow F_{i}} \operatorname{DM}\left(\prod X_{i}\right)
$$

is an equivalence for cofinality reasons. This shows the claim for DM*.

The one for $\mathrm{DM}^{!}$(and placid prestacks, or arbitrary ones for $S$ being a field) is done the same way, using Lemma A.9 instead.

Corollary A.15 The restriction of $\mathrm{DM}^{!}$to $\left(\operatorname{PreStk}_{S}^{\mathrm{pl}}\right)^{\mathrm{op}}$ is symmetric lax monoidal. In particular, for any two placid prestacks $X_{1}, X_{2}$, there is a natural functor

$$
\operatorname{DM}\left(X_{1}\right) \otimes \operatorname{DM}\left(X_{2}\right) \rightarrow \operatorname{DM}\left(X_{1} \times_{S} X_{2}\right)
$$

Proof The restriction of $\mathrm{DM}^{!}$to $\operatorname{PreStk}_{S}^{\mathrm{pl}}$ is the unique colimit-preserving functor extending the restriction of $\mathrm{DM}^{!}$to $\left(\mathrm{AffSch}_{S}^{\mathrm{pl}}\right)^{\text {op }}$. This latter functor is the unique extension, preserving ( $\kappa$-small) cofiltered limits, of the restriction of $\mathrm{DM}^{!}$to $\left(\mathrm{AffSch}_{S, \mathrm{sm}}^{\mathrm{ft}}\right)^{\mathrm{op}}$. We can conclude using Corollary A.14.

\section{References}

1. Ayoub, J., Gallauer, M., Vezzani, A.: The six-functor formalism for rigid analytic motives (2020). arXiv:2010.15004v1 
2. Ayoub, J.: Les six opérations de Grothendieck et le formalisme des cycles évanescents dans le monde motivique. I. Astérisque (314), x+466 pp. (2008) (2007)

3. Ayoub, J.: Les six opérations de Grothendieck et le formalisme des cycles évanescents dans le monde motivique. II. Astérisque (315), vi+364 pp. (2008) (2007)

4. Ayoub, J.: The motivic vanishing cycles and the conservation conjecture. In: Algebraic Cycles and Motives, vol. 1, London Math. Soc. Lecture Note Ser., vol. 343, pp. 3-54. Cambridge Univ. Press, Cambridge (2007) https://doi.org/10.1017/CBO9780511721496.002

5. Ayoub, J.: La réalisation étale et les opérations de Grothendieck (2014) https://doi.org/10.24033/asens. 2210

6. Barwick, C., Glasman, S., Nardin, D.: Dualizing cartesian and cocartesian fibrations (2014). arXiv: 1409.2165

7. Baumann, P., Riche, S.: Notes on the geometric Satake equivalence. In: Relative Aspects in Representation Theory, Langlands Functoriality and Automorphic Forms, Lecture Notes in Math., vol. 2221, pp. 1-134. Springer, Cham (2018)

8. Beilinson, A., Drinfeld, V.: Quantization of Hitchin's integrable system and Hecke eigensheaves (1999). http://www.math.utexas.edu/users/benzvi/Langlands.html

9. Bondarko, M.V.: Weight structures and 'weights' on the hearts of $t$-structures. Homol. Homot. Appl. 14(1), 239-261 (2012). https://doi.org/10.4310/HHA.2012.v14.n1.a12

10. Bondarko, M.V.: Weights for relative motives: relation with mixed complexes of sheaves. Int. Math. Res. Not. IMRN 17, 4715-4767 (2014). https://doi.org/10.1093/imrn/rnt088

11. Bourbaki, N.: Éléments de mathématique. Fasc. XXXIV. Groupes et algèbres de Lie. Chapitre IV: Groupes de Coxeter et systèmes de Tits. Chapitre V: Groupes engendrés par des réflexions. Chapitre VI: systèmes de racines. Actualités Scientifiques et Industrielles, No. 1337. Hermann, Paris (1968)

12. Bruhat, F., Tits, J.: Groupes réductifs sur un corps local. II. Schémas en groupes. Existence d'une donnée radicielle valuée. Inst. Hautes Études Sci. Publ. Math. (60):197-376 (1984). http://www.numdam.org/ item?id=PMIHES_1984_60_5_0

13. Buzzard, K., Gee, T.: The conjectural connections between automorphic representations and Galois representations. In: Automorphic Forms and Galois Representations, vol. 1, London Math. Soc. Lecture Note Ser., vol. 414, pp. 135-187. Cambridge Univ. Press, Cambridge (2014). https://doi.org/10.1017/ CBO9781107446335.006

14. Cisinski, D.-C., Déglise, F.: Étale motives. Compos. Math. 152(3), 556-666 (2016). https://doi.org/ 10.1112/S0010437X15007459

15. Cisinski, D.-C., Déglise, F.: Triangulated categories of mixed motives. Springer Monographs in Mathematics. Springer, Cham (2019). arXiv:0912.2110. https://doi.org/10.1007/978-3-030-33242-6

16. Cisinski, D.-C.: Cohomological methods in intersection theory. arXiv:1905.03478 (2019)

17. Conrad, B., Gabber, O., Prasad, G.: Pseudo-reductive Groups, New Mathematical Monographs, vol. 26, 2nd edn. Cambridge University Press, Cambridge (2015). https://doi.org/10.1017/ CBO9781316092439

18. Conrad, B.: Reductive group schemes. In: Autour des schémas en groupes, vol. I, Panor. Synthèses, vol. 42/43, pp. 93-444. Soc. Math. France, Paris (2014)

19. Deligne, P.: La conjecture de Weil. II. Inst. Hautes Études Sci. Publ. Math. 52, 137-252 (1980)

20. Deligne, P.: Letter to Serre (2007). https://publications.ias.edu/sites/default/files/2007\%20Serre_0.pdf

21. Deligne, P., Milne, J.S., Ogus, A., Shih, K.: Hodge Cycles, Motives, and Shimura Varieties. Lecture Notes in Mathematics, vol. 900. Springer, Berlin (1982)

22. Elmanto, E., Khan, A.A.: Perfection in motivic homotopy theory. Proc. Lond. Math. Soc. 120(1), 28-38 (2020). https://doi.org/10.1112/plms.12280. arXiv:1812.07506

23. Fargues, L., Scholze, P.: Geometrization of the local Langlands correspondence (2021). arXiv:2102.13459

24. Frenkel, E., Gross, B.: A rigid irregular connection on the projective line. Ann. Math. (2) 170(3), 1469-1512 (2009). https://doi.org/10.4007/annals.2009.170.1469

25. Fontes, E.E.: A weighty theorem of the heart for the algebraic $K$-theory of higher categories. $\mathrm{PhD}$ thesis at the University of Texas at Austin (2017)

26. Gaitsgory, D.: Construction of central elements in the affine Hecke algebra via nearby cycles. Invent. Math. 144(2), 253-280 (2001). https://doi.org/10.1007/s002220100122

27. Gaitsgory, D.: On de Jong's conjecture. Isr. J. Math. 157, 155-191 (2007). https://doi.org/10.1007/ s11856-006-0006-2 
28. Gaitsgory, D.: The local and global versions of the Whittaker category (2019). http://www.math. harvard.edu/ gaitsgde/GL/LocWhit.pdf

29. Gaitsgory, D., Rozenblyum, N.: A Study in Derived Algebraic Geometry, vol. I. Correspondences and Duality, Mathematical Surveys and Monographs, vol. 221. American Mathematical Society, Providence (2017)

30. Ginzburg, V.: Perverse sheaves on a Loop group and Langlands' duality (2000). arXiv:alg-geom/9511007

31. Glasman, S.: Day convolution for $\infty$-categories. Math. Res. Lett. 23(5), 1369-1385 (2016). https:// doi.org/10.4310/MRL.2016.v23.n5.a6. arXiv:1308.4940

32. Gross, B.H.: Groups over Z. Invent. Math. 124(1-3), 263-279 (1996). https://doi.org/10.1007/ s002220050053

33. Gross, B.H.: On the Satake isomorphism. In: Galois Representations in Arithmetic Algebraic Geometry (Durham, 1996), London Math. Soc. Lecture Note Ser., vol. 254, pp. 223-237. Cambridge Univ. Press, Cambridge (1998). https://doi.org/10.1017/CBO9780511662010.006

34. Haines, T.: A proof of the Kazhdan-Lusztig purity theorem via the decomposition theorem of BBD

35. Haines, T.J., Richarz, T.: The test function conjecture for parahoric local models (2018). arXiv: 1801.07094

36. Hébert, D.: Structure de poids à la Bondarko sur les motifs de Beilinson. Compos. Math. 147(5), 1447-1462 (2011). arXiv:1007.0219. https://doi.org/10.1112/S0010437X11005422

37. Heinloth, J., Ngô, B.-C., Yun, Z.: Kloosterman sheaves for reductive groups. Ann. Math. (2) 177(1), 241-310 (2013). https://doi.org/10.4007/annals.2013.177.1.5

38. Hoyois, M.: The six operations in equivariant motivic homotopy theory. Adv. Math. 305, 197-279 (2017). https://doi.org/10.1016/j.aim.2016.09.031

39. Jantzen, J.C.: Representations of algebraic groups. Pure and Applied Mathematics, vol. 131. Academic Press, Boston (1987)

40. Jin, F., Yang, E.: Künneth formulas for motives and additivity of traces (2018). arXiv:1812.06441

41. Kazhdan, D., Lusztig, G.: Representations of Coxeter groups and Hecke algebras. Invent. Math. 53(2), 165-184 (1979). https://doi.org/10.1007/BF01390031

42. Kazhdan, D., Lusztig, G.: Schubert varieties and Poincaré duality. In: Geometry of the Laplace operator (Proc. Sympos. Pure Math., Univ. Hawaii, Honolulu, Hawaii, 1979), Proc. Sympos. Pure Math., vol. XXXVI, pp. 185-203. Amer. Math. Soc., Providence (1980)

43. Khan, A.: Motivic homotopy theory in derived algebraic geometry. $\mathrm{PhD}$ thesis, Universität Duisburg-Essen (2016). https://duepublico.uni-duisburg-essen.de/servlets/DerivateServlet/Derivate42021/Diss_Khan.pdf

44. Kiehl, R., Weissauer, R.: Weil conjectures, perverse sheaves and $l$ 'adic Fourier transform, Ergebnisse der Mathematik und ihrer Grenzgebiete. 3. Folge, vol. 42. A Series of Modern Surveys in Mathematics [Results in Mathematics and Related Areas. 3rd Series. A Series of Modern Surveys in Mathematics]. Springer, Berlin (2001). https://doi.org/10.1007/978-3-662-04576-3

45. Krause, H.: Krull-Schmidt categories and projective covers. Expo Math. 33(4), 535-549 (2015). https:// doi.org/10.1016/j.exmath.2015.10.001

46. Lafforgue, V.: Chtoucas pour les groupes réductifs et paramétrisation de Langlands globale. J. Am. Math. Soc. 31(3), 719-891 (2018). https://doi.org/10.1090/jams/897

47. Levine, M.: Tate motives and the vanishing conjectures for algebraic $K$-theory. In Algebraic $K$-theory and algebraic topology (Lake Louise, AB, 1991), NATO Adv. Sci. Inst. Ser. C Math. Phys. Sci., vol. 407, pp. 167-188. Kluwer Acad. Publ., Dordrecht (1993)

48. Lurie, J.: Higher topos theory. Annals of Mathematics Studies, vol. 170. Princeton University Press, Princeton (2009)

49. Lurie, J.: Higher Algebra (2017). http://www.math.harvard.edu/ lurie/

50. Lusztig, G.: Singularities, character formulas, and a $q$-analog of weight multiplicities. In: Analysis and Topology on Singular Spaces. II, III (Luminy, 1981), Astérisque, vol. 101, pp. 208-229. Soc. Math. France, Paris (1983)

51. Lusztig, G., Yun, Z.: A ( $-q)$-analogue of weight multiplicities. J. Ramanujan Math. Soc. 28A, 311-340 (2013)

52. Mirković, I., Vilonen, K.: Geometric Langlands duality and representations of algebraic groups over commutative rings. Ann. Math. (2) 166(1), 95-143 (2007)

53. Nikolaus, T.: Stable $\infty$-operads and the multiplicative Yoneda lemma (2016). arXiv:1608.02901 
54. Pappas, G., Zhu, X.: Local models of Shimura varieties and a conjecture of Kottwitz. Invent. Math. 194(1), 147-254 (2013). https://doi.org/10.1007/s00222-012-0442-z

55. Quillen, D.: On the cohomology and $K$-theory of the general linear groups over a finite field. Ann. Math. 2(96), 552-586 (1972)

56. Raskin, S.: D-modules on infinite dimensional varieties. Preprint, available at the web site of the author

57. Richarz, T.: A new approach to the geometric Satake equivalence. Doc. Math. 19, 209-246 (2014). arXiv:1207.5314v2

58. Richarz, T.: On the Iwahori Weyl group. Bull. Soc. Math. Fr. 144(1), 117-124 (2016)

59. Richarz, T., Scholbach, J.: Tate motives on Witt vector affine flag varieties (2020). arXiv:2003.02593

60. Richarz, T., Scholbach, J.: The intersection motive of the moduli stack of shtukas. Forum Math. (Sigma) 8(e8) (2020). arXiv:1901.04919

61. Richarz, T., Zhu, X.: Appendix to: The geometric Satake correspondence for ramified groups. Ann. Sci. Éc. Norm. Supér. (2) 48(2), 409-451 (2015). https://doi.org/10.24033/asens.2248

62. Robalo, M.: Théorie Homotopique Motivique des Espaces Noncommutatifs. PhD thesis at the Université Montpellier 2 (2014). https://webusers.imj-prg.fr/ marco.robalo/these.pdf

63. Soergel, W., Wendt, M.: Perverse motives and graded derived category mathcalO. J. Inst. Math. Jussieu 17(2), 347-395 (2018). https://doi.org/10.1017/S1474748016000013. arXiv:1404.6333v3

64. The Stacks Project Authors. Stacks Project. http://stacks.math.columbia.edu (2017)

65. Zhu, X.: Affine Grassmannians and the geometric Satake in mixed characteristic. Ann. Math. (2) 185(2), 403-492 (2017). https://doi.org/10.4007/annals.2017.185.2.2

66. Zhu, X.: An introduction to affine Grassmannians and the geometric Satake equivalence. In: Geometry of Moduli Spaces and Representation Theory, IAS/Park City Math. Ser., vol. 24, pp. 59-154. Amer. Math. Soc., Providence (2017)

67. Zhu, X.: Geometric Satake, categorical traces, and arithmetic of Shimura varieties. In: Current Developments in Mathematics 2016, pp. 145-206. Int. Press, Somerville (2018)

68. Zhu, X.: A note on integral Satake isomorphisms (2021). arXiv:2005.13056

Publisher's Note Springer Nature remains neutral with regard to jurisdictional claims in published maps and institutional affiliations. 